Aus der Abteilung Psychiatrie und Psychotherapie

(Prof. Dr. med. P. Falkai)

im Zentrum Psychosoziale Medizin

der Medizinischen Fakultät der Universität Göttingen

\title{
Der Einfluss von Ziprasidon auf den Schlaf und die Kortisolexkretion
}

\author{
INAUGURAL-DISSERTATION \\ zur Erlangung des Doktorgrades \\ der Medizinischen Fakultät \\ der Georg-August-Universität zu Göttingen
}

vorgelegt von

Anna-Catharina Hilda Neumann

aus Norden

Göttingen, 2007 
Dekan: Prof. Dr. med. C. Frömmel

I. Berichterstatter: Priv.-Doz. Dr. med. S. Cohrs

II. Berichterstatter/in:

III. Berichterstatter/in:

Tag der mündlichen Prüfung: 


\section{Inhaltsverzeichnis}

Abkürzungen iii

Tabellenverzeichnis vi

Abbildungsverzeichnis vii

1 Einleitung und Überblick 1

2 Grundlagen 3

2.1 Schizophrenie und Depression ... . . . . . . . . . . 3

2.1.1 Unterschied zwischen klassischen und atypischen Antipsychotika 4

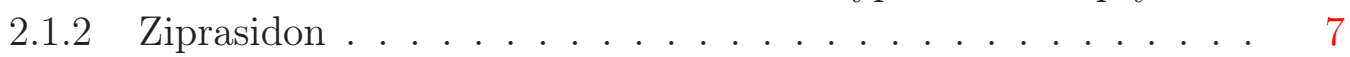

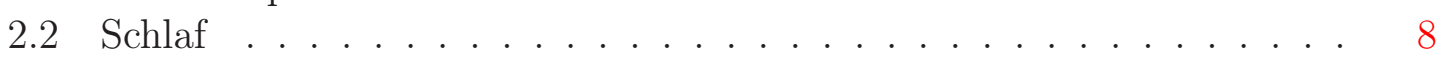

2.2.1 Schlaf bei gesunden Probanden . . . . . . . . . . . . 9

2.2.2 Funktion und Regulation des Schlafs . . . . . . . . . . . . 11

2.2.3 Schlaf bei depressiven Patienten . . . . . . . . . . . . . . . 14

2.2.4 Schlaf bei schizophrenen Patienten . . . . . . . . . . . 15

2.2.5 Hypothesen zum Schlaf bei Depression und Schizophrenie . . . 17

2.2.6 Zusammenhang zwischen Schlaf und Symptomatik bei Schizophrenie . . . . . . . . . . . . . . . . . . 19

2.3 Wirkung der Antipsychotika auf den Schlaf . . . . . . . . . . . . . 19

2.3.1 Wirkung klassischer Antipsychotika bei schizophrenen Patienten 20

2.3.2 Wirkung atypischer Antipsychotika bei schizophrenen Patienten 21

2.3.3 Wirkung klassischer Antipsychotika bei gesunden Probanden . 22

2.3.4 Wirkung atypischer Antipsychotika bei gesunden Probanden . 24

2.4 Hypothalamus-Hypophysen-Nebennieren-Achse . . . . . . . . . . . 25

2.4.1 HPA-Achse bei Depression und bei Schizophrenie . . . . . . . 26

2.4.2 Zusammenhang zwischen HPA-Achse und Symptomatik bei

Schizophrenie . . . . . . . . . . . . . . . 27

2.5 Wirkung der Antipsychotika auf die HPA-Achse . . . . . . . . . . . . 29

2.5.1 Wirkung klassischer Antipsychotika bei schizophrenen Patienten 30

2.5.2 Wirkung atypischer Antipsychotika bei schizophrenen Patienten 30

2.5.3 Wirkung klassischer Antipsychotika bei gesunden Probanden . 31

2.5.4 Wirkung atypischer Antipsychotika bei gesunden Probanden . 32 
2.6 Fragestellung . . . . . . . . . . . . . . . . . . . 32

3 Methodik 36

3.1 Art und Ort der Studie . . . . . . . . . . . . . . . . . . 36

3.2 Untersuchungspopulation . . . . . . . . . . . . . . . . . 36

3.3 Einschluss-, Ausschluss- und Abbruchkriterien . . . . . . . . . . . 36

3.4 Ethik . . . . . . . . . . . . . . . . . . . . 38

3.5 Studienablauf . . . . . . . . . . . . . . . . 38

3.5.1 Prüfmedikation . . . . . . . . . . . . . . . . . . 39

3.5 .2 Insomnie-Modell . . . . . . . . . . . . . . . . . . . . 40

3.5.3 Fraktionierte Urinsammlung . . . . . . . . . . . . . . . . 40

3.6 Sicherheitsverlaufsparameter . . . . . . . . . . . . . . . . . . . 41

3.7 Untersuchungsverfahren . . . . . . . . . . . . . . . . . . 41

3.7.1 Beurteilung der objektiven Schlafqualität (Polysomnographie) 41

3.7.2 Beurteilung der subjektiven Schlafqualität (Fragebögen) . . . 42

3.7.3 Kortisolbestimmung im Urin . . . . . . . . . . . . . . . . 44

3.8 Statistik . . . . . . . . . . . . . . . . . . . 44

4 Ergebnisse 46

4.1 Demographische Daten . . . . . . . . . . . . . . . . . . 46

4.2 Erfasste Schlafparameter . . . . . . . . . . . . . . . . . . 46

4.2.1 Objektive Schlafparameter . . . . . . . . . . . . . . . . . 46

4.2.2 Subjektive Schlafparameter . . . . . . . . . . . . . . . . . . 49

4.3 Kortisolexkretion . . . . . . . . . . . . . . 51

5 Diskussion 54

5.1 Schlaf . . . . . . . . . . . . . . . . . 54

5.1 Einfluss von Ziprasidon auf den Schlaf . . . . . . . . . . . 55

5.1.2 Vergleich der Wirkung Ziprasidons mit der der Antidepressiva 57

5.1.3 Interpretation der Wirkung Ziprasidons auf den Schlaf . . . . 59

5.2 Kortisolexkretion . . . . . . . . . . . . . . . . 60

5.2.1 Einfluss von Ziprasidon auf die HPA-Achse . . . . . . . . . . 62

5.2.2 Interpretation der Wirkung Ziprasidons auf die Kortisolexkretion 63

5.3 Ausblick . . . . . . . . . . . . . . . . . 66 66

6 Zusammenfassung $\quad 68$

$\begin{array}{ll}\text { Anhang } & 71\end{array}$

$\begin{array}{ll}\text { Literaturverzeichnis } & 78\end{array}$ 


\section{Abkürzungen}

\begin{tabular}{|c|c|}
\hline Abkürzung & Bedeutung \\
\hline $\mathrm{ACTH}$ & $\begin{array}{l}\text { Adrenocorticotropic hormone } \\
\text { Adrenokortikotropes Hormon }\end{array}$ \\
\hline AHI & Apnoe-Hypopnoe-Index \\
\hline ALT & Alaninaminotransferase \\
\hline ANOVA & Analysis of variance \\
\hline AST & Aspartataminotransferase \\
\hline Anz. Aufwachv. & Anzahl der Aufwachvorgänge \\
\hline \multirow[t]{2}{*}{$\mathrm{CRH}$} & Corticotropin-releasing hormone \\
\hline & Kortikotropin-freisetzendes Hormon \\
\hline EDTA & $\begin{array}{l}\text { Ethylenediaminetetraacetic acid } \\
\text { Ethylendiamintetraessigsäure }\end{array}$ \\
\hline EEG & Elektroenzephalogramm \\
\hline $\mathrm{EKG}$ & Elektrokardiogramm \\
\hline EMG & Elektromyogramm \\
\hline EOG & Elektrookulogramm \\
\hline FSUCL & $\begin{array}{l}\text { Fischer Somatische Symptome oder Unerwünschte Effekte } \\
\text { Check List }\end{array}$ \\
\hline GABA & $\gamma$-Aminobuttersäure \\
\hline GES & Gefühl des Erholtseins nach dem Schlaf \\
\hline$\gamma-\mathrm{GT}$ & $\gamma$-Glutamyltranspeptidase \\
\hline \multirow[t]{2}{*}{ HPA-Achse } & Hypothalamic-pituitary-adrenal axis \\
\hline & Hypothalamus-Hypophysen-Nebennieren-Achse \\
\hline \multirow[t]{2}{*}{ 5-HT-Rezeptor } & 5-Hydroxy-Tryptamin-Rezeptor \\
\hline & Serotonin-Rezeptor \\
\hline \multirow[t]{2}{*}{ M. (Mm.) } & Musculus (Musculi) \\
\hline & Muskel (Muskeln) \\
\hline \multirow[t]{2}{*}{$\mathrm{MCH}$} & Mean corpuscular haemoglobin \\
\hline & Mittlere Hämoglobinmenge pro Erythrozyt \\
\hline \multirow[t]{2}{*}{$\mathrm{MCHC}$} & Mean corpuscular haemoglobin concentration \\
\hline & Mittlere Hämoglobinkonzentration des Einzelerythrozyten \\
\hline
\end{tabular}




\begin{tabular}{|c|c|}
\hline Abkürzung & Bedeutung \\
\hline \multirow[t]{2}{*}{$\mathrm{MCV}$} & Mean corpuscular volume \\
\hline & Mittleres Erythrozyteneinzelvolumen \\
\hline \multirow[t]{2}{*}{ MT } & Movement time \\
\hline & Bewegungszeit \\
\hline MW & Mittelwert \\
\hline n. b. & nicht berichtet \\
\hline NREM & Non-Rapid eye movement \\
\hline \multirow[t]{2}{*}{ PLMS } & Periodic leg movements in sleep \\
\hline & Anzahl periodischer Beinbewegungen im Schlaf \\
\hline \multirow{2}{*}{ PMS-Index } & Periodic movements in sleep-Index \\
\hline & Index der periodischen Beinbewegungen im Schlaf \\
\hline PSS & Psychosomatische Symptome in der Schlafphase \\
\hline PSYAA & Psychische Ausgeglichenheit am Abend \\
\hline PSYEA & Psychische Erschöpftheit am Abend \\
\hline \multirow[t]{2}{*}{ REM } & Rapid eye movement \\
\hline & Schnelle Augenbewegungen \\
\hline REM-Dichte-1 & REM-Dichte der ersten REM-Periode \\
\hline REM-L & REM-Latenz \\
\hline \multirow[t]{2}{*}{$\mathrm{SD}$} & Standard deviation \\
\hline & Standardabweichung \\
\hline \multirow[t]{2}{*}{$\mathrm{SE}$} & Sleep efficiency \\
\hline & Schlafeffizienz \\
\hline $\mathrm{SF}-\mathrm{A}$ & Schlaffragebogen-A \\
\hline \multirow[t]{2}{*}{ SL } & Sleep latency \\
\hline & Schlaflatenz \\
\hline \multirow[t]{2}{*}{$\mathrm{SNRI}(\mathrm{s})$} & Selective noradrenalin reuptake inhibitor(s) \\
\hline & Selektive(r) Noradrenalin-Wiederaufnahme-Hemmer \\
\hline \multirow[t]{2}{*}{ SPT } & Sleep period time \\
\hline & Schlafperiodendauer \\
\hline \multirow[t]{2}{*}{$\mathrm{SSRI}(\mathrm{s})$} & Selective serotonin reuptake inhibitor(s) \\
\hline & Selektive(r) Serotonin-Wiederaufnahme-Hemmer \\
\hline SQ & Schlafqualität \\
\hline St. & Stadium \\
\hline Subj. & Subjektiv \\
\hline Syn. & Synonym \\
\hline \multirow[t]{2}{*}{ SWS } & Slow wave sleep \\
\hline & Tiefschlaf \\
\hline \multirow[t]{2}{*}{ TIB } & Time in bed \\
\hline & Bettzeit \\
\hline
\end{tabular}




\begin{tabular}{ll} 
Abkürzung & Bedeutung \\
\hline TST & Total sleep time \\
& Gesamtschlafzeit \\
VIS-M & Visuelle Analogskala morgens \\
vgl. & vergleiche \\
vs. & versus \\
& gegen \\
Z.n. & Zustand nach
\end{tabular}




\section{Tabellenverzeichnis}

3.1 Schematische Darstellung des Studienablaufs . . . . . . . . . . . . . . 39

3.2 Standardschlafparameter und ihre Definition . . . . . . . . . . . . 43

4.1 Polysomnographisch erfasste Schlafparameter unter Angabe des Medikations-, Stress- und Interaktionseffekts . . . . . . . . . . . . 47

4.2 Subjektive Schlafparameter unter Angabe des Medikations-, Stressund Interaktionseffekts . . . . . . . . . . . . . . . . . . 50

4.3 Kortisolkonzentration und -menge unter Angabe des Medikations-, Stress- und Interaktionseffekts . . . . . . . . . . . . . . . . . 52

5.1 Auswirkungen atypischer Antipsychotika auf den Schlaf . . . . . . . 56

5.2 Schlafstörungen bei Schizophrenie und Depression im Vergleich zur Wirkung von Ziprasidon . . . . . . . . . . . . . . . . 57

5.3 Auswirkungen atypischer Antipsychotika auf den Kortisolspiegel . . . 63

A.1 Gesamtübersicht der gemessenen Schlafparameter, Teil 1 . . . . . . . 72

A.2 Gesamtübersicht der gemessenen Schlafparameter, Teil 2 . . . . . . . 73

A.3 Gesamtübersicht der gemessenen Schlafparameter, Teil 3 . . . . . . . 74

A.4 Mittelwerte der REM-Parameter über Nacht 1 und Nacht 2 . . . . . . 75

A.5 Gesamtübersicht der pro Sammelzeitraum gemessenen Kortisolexkretion ........................... 76

A.6 Mittelwerte der Kortisolexkretion über Nacht 1 und Nacht 2 . . . . . 77 


\section{Abbildungsverzeichnis}

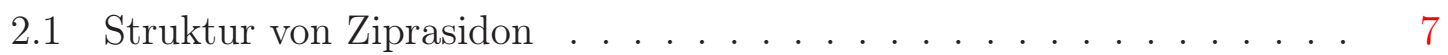

2.2 Rezeptor-Bindungsprofil von Ziprasidon . . . . . . . . . . . . . . 8

2.3 Schlafstruktur einer jungen gesunden Person . . . . . . . . . . . . . . 11

2.4 Reziprokes Interaktionsmodell . . . . . . . . . . . . . . . . . . 14

2.5 Zwei-Prozess-Modell . . . . . . . . . . . . . . . . . . . . . . . 15

2.6 Schlafstruktur eines Depressiven . . . . . . . . . . . . . . . 16

4.1 Effekt von Ziprasidon auf den REM-Schlaf . . . . . . . . . . . . . . . 48

4.2 Effekt von Ziprasidon auf die Kortisolexkretion . . . . . . . . . . . . . 53 


\section{Kapitel 1}

\section{Einleitung und Überblick}

In der Statistik der Deutschen Rentenversicherung über Rentenzugänge 2005 wegen verminderter Erwerbsfähigkeit stellen psychische Erkrankungen mit 38,4\% den weitaus größten Anteil dar. Erst an zweiter Stelle folgen Krankheiten des MuskelSkelett-Systems und des Bindegewebes mit 17,8\% (Deutsche Rentenversicherung, 2006). In Anbetracht dieser Zahlen ist die richtige Behandlung psychischer Erkrankungen von großer Bedeutung.

Die Ursache wichtiger psychiatrischer Krankheitsbilder, wie beispielsweise Schizophrenie und Depression, erklärt man durch das Zusammenwirken multifaktorieller Komponenten. Von Bedeutung ist dabei insbesondere eine individuelle genetische Disposition, die mit äußeren Lebensumständen und bisherigen Erfahrungen in Beziehung tritt. Die genauen pathophysiologischen Zusammenhänge sind jedoch bis heute nicht vollständig geklärt (Möller et al., 2001).

Mittlerweile gibt es eine Reihe von wirksamen Antidepressiva und Antipsychotika zur Behandlung der entsprechenden Erkrankungen. Auch die Wirkungsweise dieser Medikamente ist nicht vollständig erforscht. Einerseits sind die Hirnstrukturen und Neurotransmittersysteme im menschlichen Hirn sehr komplex verwoben und schwierig zu untersuchen, anderseits können auch die Krankheitsbilder individuell sehr unterschiedlich ausgeprägt sein.

Schizophrenie und Depression gehen in den meisten Fällen mit Schlafstörungen einher, teilweise gehen diese den Erkrankungen sogar voraus oder sind Anzeichen eines Rückfalls. Anhand polysomnographischer Untersuchungen kann gezeigt werden, dass der Schweregrad der Schizophrenie, das Ausmaß der Negativsymptomatik und ein schlechterer Krankheitsverlauf mit dem Anteil bestimmter Schlafstadien korreliert (Benson und Zarcone, 2000; Ganguli et al., 1987; Kempenaers et al., 1988; Keshavan et al., 1995a; Tandon et al., 1992, 2000).

Darüber hinaus hat man bei schizophrenen Patienten eine Überaktivierung der Hypothalamus-Hypophysen-Nebennieren-Achse (Hypothalamic-pituitary-adrenal axis, 
HPA-Achse) festgestellt. Dies drückt sich beispielsweise durch einen erhöhten basalen Kortisolspiegel im Blut aus. Es besteht eine Assoziation zwischen der erhöhten Aktivität der HPA-Achse und der Ausprägung von Negativsymptomen, depressiven Symptomen, kognitiven Defiziten und internistischen Begleiterkrankungen (Jones et al., 1994; Ryan et al., 2004; Thakore et al., 2002; Walder et al., 2000; Zhang et al., 2005).

Im Gegensatz zu klassischen Antipsychotika zeichnen sich die atypischen Antipsychotika durch eine geringere Neigung zur Induktion extrapyramidal-motorischer Bewegungsstörungen und durch eine bessere Wirkung auf Negativsymptome, depressive Symptome sowie kognitive Defizite aus. Damit wirken sie auf einige Symptome, die mit einer Überaktivierung der HPA-Achse und mit einem gestörten Schlaf in Zusammenhang gebracht werden. Obwohl dieser Zusammenhang besteht, sind die Auswirkungen atypischer Antipsychotika auf die HPA-Achse und auf den Schlaf bisher kaum studiert worden.

Bei der Untersuchung der Wirkung von Antipsychotika auf den Schlaf und die HPAAchse schizophrener Personen ist es schwierig zu beurteilen, welche Effekte primäre Arzneimittelwirkungen darstellen und welche Effekte als sekundäre Arzneimittelwirkungen im Rahmen einer gebesserten Symptomatik zu interpretieren sind. Um direkte Arzneimitteleffekte untersuchen zu können, sind Studien an gesunden Versuchspersonen nötig. Aus diesem Grund sind in die vorliegende Studie nur gesunde Probanden eingeschlossen worden. Die Schlafstörung und Erhöhung des Kortisolspiegels, wie sie unter anderem bei schizophrenen Patienten vorkommt, wurde bei den Probanden in einer Untersuchungsbedingung durch akustischen Stress provoziert.

Das Ziel dieser Studie war, die Auswirkungen von Ziprasidon, einem atypischen Antipsychotikum, auf den Schlaf und die Kortisolexkretion im Vergleich zu Plazebo zu erfassen. Sowohl unter Ruhebedingungen als auch unter akustischem Stress wurde der Schlaf polysomnographisch abgeleitet. Die Kortisolexkretion wurde im fraktioniert gesammelten Urin untersucht.

Die vorliegende Dissertationsschrift ist folgendermaßen gegliedert: In Kapitel 2 wird auf die theoretischen Grundlagen eingegangen. Zunächst werden die psychischen Erkrankungen Schizophrenie und Depression erläutert. Der Unterschied zwischen klassischen und atypischen Antipsychotika wird dargelegt. Ferner wird das atypische Antipsychotikum Ziprasidon vorgestellt. Die folgenden Abschnitte beleuchten den Schlaf und die Auswirkungen von Antipsychotika auf den Schlaf gesunder Probanden und schizophrener Patienten. Anschließend werden die HPA-Achse und der Einfluss von Antipsychotika auf die HPA-Achse betrachtet. Am Ende des Kapitels wird die Fragestellung dieser Arbeit zusammengefasst. Die Methodik der durchgeführten Studie wird in Kapitel 3 dargelegt. Im Anschluss werden die Ergebnisse in Kapitel 4 präsentiert und vor dem Hintergrund der vorhandenen Literatur in Kapitel 5 diskutiert. 


\section{Kapitel 2}

\section{Grundlagen}

\subsection{Schizophrenie und Depression}

Die Schizophrenie ist eine komplexe Störung des Denkens, Fühlens und Wollens und kann in individuell unterschiedlicher Form von Positivsymptomen, wie z. B. Wahnvorstellungen und Halluzinationen, bzw. Negativsymptomen, wie z. B. Antriebsmangel und Affektarmut, geprägt sein (Möller et al., 2001).

Viele Arbeiten haben sich mit den pathophysiologischen Mechanismen dieser Erkrankung befasst. In den 1960ern postulierten Wissenschaftler die sogenannte DopaminHypothese, bei der man von einer Überaktivität dopaminerger Strukturen im Hirn bei der Schizophrenie ausging. Vielfach ist auch eine erhöhte Dopamin-Rezeptordichte diskutiert worden. Auch eine erhöhte Rezeptorsensitivität spielt möglicherweise eine tragende Rolle in der Entstehung und Aufrechterhaltung einer Schizophrenie (Laruelle et al., 1996).

Neueren Datums sind Studien, die eine kortikal-subkortikale Dopamin-Imbalance belegen (Abi-Dargham, 2004). Gemäß dieser Theorie werden die Positivsymptome vorwiegend auf einen Dopamin-Überschuss in subkortikalen Hirnarealen zurückgeführt. Die Negativsymptome und kognitiven Einschränkungen führt man dagegen auf einen Dopaminmangel in kortikalen Bereichen zurück (Abi-Dargham, 2004).

Eine andere wichtige psychiatrische Erkrankung ist die Depression. Sie ist eine affektive Störung, die hauptsächlich mit gedrückter Stimmung, Antriebsmangel und Konzentrationsschwierigkeiten einhergeht. Auch über die pathophysiologischen Ursachen der Depression ist viel geforscht worden. Eine Theorie ist beispielsweise die Monoamin-Hypothese, die von einem Mangel der Neurotransmitter Serotonin, Noradrenalin und/oder Dopamin ausgeht (Delgado, 2000). Diese wurde inzwischen modifiziert und man geht davon aus, dass auch bei dieser Erkrankung Veränderungen der Dichte und Empfindlichkeit von Rezeptoren vorliegen (Möller et al., 2001). 
Gegen eine Depression werden im Rahmen einer Pharmakotherapie Antidepressiva angewendet. Man unterscheidet eine Reihe verschiedener Wirkstoffklassen.

Eine Einteilung erfolgt zum einen nach ihrer chemischen Struktur in tri- bzw. tetrazyklische Antidepressiva und chemisch neuartige Antidepressiva. Eine andere Einteilung orientiert sich an dem zentralen Wirkmechanismus im Hirn. Demzufolge gibt es selektive Serotonin-Wiederaufnahme-Hemmer (Selective serotonin reuptake inhibitor(s), SSRI(s)), selektive Noradrenalin-Wiederaufnahme-Hemmer (Selective noradrenalin reuptake inhibitor(s), SNRI(s)), überwiegende Serotonin- bzw. Noradrenalin-Wiederaufnahme-Hemmer, kombinierte Serotonin- und Noradrenalin-WiederaufnahmeHemmer, Monoaminooxidasehemmer und andere Wirkmechanismen (Benkert und Hippius, 2003).

Laut Arzneimittel-Report 2006 werden in Deutschland am häufigsten die trizyklischen Antidepressiva und in zunehmendem Maße auch die SSRIs verordnet (Glaeske und Janhsen, 2006).

\subsubsection{Unterschied zwischen klassischen und atypischen Antipsychotika}

Zur Behandlung einer Schizophrenie werden Antipsychotika eingesetzt. Die Antipsychotika werden häufig synonym als Neuroleptika bezeichnet. Dies hat vor allem historische Gründe. So beschrieben Delay und Deniker Anfang der 1950er Jahre einen durch Injektion von Chlorpromazin hervorgerufenen Zustand, der durch herabgesetzte motorische Aktivität, affektive Indifferenz und emotionale Neutralität gekennzeichnet war. Dies bezeichneten sie als „neuroleptisches Syndrom“(López-Muñoz et al., 2004). Um auf diesen Zustand einzugehen, hat man die Substanzklasse als Neuroleptika bezeichnet. Die neueren Medikamente weisen insbesondere die motorischen Effekte bei normaler Dosierung nicht mehr auf, sondern kennzeichnen sich vor allem durch ihre therapeutische Wirkung bei schizophrenen Psychosen. Aus diesem Grund ist es heute richtiger, von Antipsychotika zu sprechen (Benkert und Hippius, 2003).

Die Antipsychotika wirken je nach Substanz antagonistisch auf verschiedene Rezeptoren. Dazu gehören die Dopamin-Rezeptoren $\left(\mathrm{D}_{1}-\mathrm{D}_{4}\right)$, Serotonin-Rezeptoren, Histamin-Rezeptoren vom Typ $\mathrm{H}_{1}$, muskarinergen Acetylcholin-Rezeptoren vom Typ $\mathrm{M}_{1}$ und die Adrenalin-Rezeptoren vom Typ $\alpha_{1}$ und $\alpha_{2}$. Einige Antipsychotika hemmen zusätzlich die Noradrenalin- und/oder Serotonin-Wiederaufnahme (Stahl und Shayegan, 2003).

Die Serotonin-Rezeptoren werden synonym als 5-Hydroxy-Tryptamin-Rezeptoren (5-HT-Rezeptoren) bezeichnet. Bisher sind sieben verschiedene Rezeptorfamilien 
(5-HT $\left.{ }_{1-7}\right)$ bekannt (Giertz et al., 1996). Die 5-HT ${ }_{1}$ und 5-HT ${ }_{2}$-Rezeptorfamilie unterteilt man jeweils noch in drei Rezeptortypen 5-HT $1 \mathrm{~A}, 5-\mathrm{HT}_{1 \mathrm{~B}}$ und $5-\mathrm{HT}_{1 \mathrm{D}}$ bzw. $5-\mathrm{HT}_{2 \mathrm{~A}}, 5-\mathrm{HT}_{2 \mathrm{~B}}$ und $5-\mathrm{HT}_{2 \mathrm{C}}$. Der $5-\mathrm{HT}_{2 \mathrm{C}}$-Rezeptor wurde zunächst fälschlicherweise als $5-\mathrm{HT}_{1 \mathrm{C}}$-Rezeptor bezeichnet, mittlerweile gilt seine Zugehörigkeit zur $5-\mathrm{HT}_{2}{ }^{-}$ Rezeptor-Familie jedoch als gesichert (Giertz et al., 1996).

Im klinischen Gebrauch teilt man die Antipsychotika in zwei Gruppen. Es gibt typische (traditionelle, klassische, konventionelle) und atypische (neuere, 2. Generation) Antipsychotika. Die atypischen Medikamente führen im Gegensatz zu den typischen zu keinen bzw. nur zu geringen extrapyramidal-motorischen Bewegungsstörungen ${ }^{1}$ und zu keiner bzw. zu einer weitaus geringeren Erhöhung des Prolaktinspiegels. Ferner besitzen sie Wirksamkeit bei den oftmals sehr therapieresistenten Negativsymptomen (Benkert und Hippius, 2003; Kane, 2003; Kapur und Mamo, 2003; Möller, 2003).

Auch wenn atypische Antipsychotika die genannten Vorteile gegenüber den klassischen Antipsychotika aufweisen, zeigen sich in vielen Fällen signifikante Gewichtszunahmen, Störungen des Glukosehaushalts und Erhöhungen des Cholesterin- bzw. Triglyzerid-Spiegels (Daniel, 2003).

Während man in den 1960er und 1970er Jahren die antipsychotische Wirkung der klassischen Antipsychotika durch ihren $\mathrm{D}_{2}$-Dopamin-Antagonismus erklärt hat, sind mit der Einführung der Antipsychotika der 2. Generation neue Erklärungsansätze nötig (Kapur und Mamo, 2003). Viele Forscher haben versucht, den klinischen Unterschied von klassischen und atypischen Antipsychotika auf der Rezeptorebene zu klären. Die Suche nach dem der Atypizität zugrunde liegenden Mechanismus ist durch die Tatsache erschwert, dass der „Prototyp“ der atypischen Antipsychotika, Clozapin, neben seinem Effekt auf den $\mathrm{D}_{2}$-Rezeptor auch Effekte an $\mathrm{D}_{1^{-}}, \mathrm{D}_{4^{-}}, \mathrm{H}_{1^{-}}, 5-\mathrm{HT}_{2^{-}}$ und muskarinergen Acetylcholin-Rezeptoren vom Typ $\mathrm{M}_{1}$ zeigt (Kapur und Mamo, 2003). Es ist jedoch davon auszugehen, dass der $\mathrm{D}_{2}$-Dopamin-Antagonismus hauptverantwortlich für auftretende extrapyramidal-motorische Wirkungen und die Erhöhung des Prolaktinspiegels ist. Die motorischen Effekte können durch gleichzeitige Blockade von 5- $\mathrm{HT}_{2 \mathrm{~A}}$-Rezeptoren und/oder muskarinergen Acetylcholin-Rezeptoren vom Typ $\mathrm{M}_{1}$ abgeschwächt werden (Richelson, 1999). Auf die verschiedenen Theorien zum Thema, welche Eigenschaften der Antipsychotika den Grad der Atypizität bestimmen, soll im Folgenden genauer eingegangen werden:

\footnotetext{
${ }^{1}$ Extrapyramidal-motorische Bewegungsstörungen sind hauptsächlich durch Muskelsteifigkeit, Muskelzittern und Bewegungsarmut gekennzeichnet. Hervorgerufen werden sie durch verminderte Aktivität oder Funktion des dopaminergen Systems. Dies kann medikamentös über einen Dopamin-Rezeptor-Antagonismus ausgelöst werden oder krankheitsbedingt im Rahmen eines Parkinson-Syndroms auftreten.
} 
Prinzipiell können die verschiedenen Theorien zwei Gruppen zugeordnet werden. Welche Theorie letztendlich richtig ist, kann bisher noch nicht abschließend geklärt werden.

Theorien der ersten Gruppe besagen, dass der Schlüssel zur Atypizität in der Wirkung auf Nicht- $\mathbf{D}_{2}$-Rezeptoren liegt. Allerdings weichen die Aussagen, welcher genaue Nicht- $\mathrm{D}_{2}$-Rezeptor der ausschlaggebende ist, voneinander ab. Die Wirkungen auf den 5-HT $\mathrm{H}_{2}$-Rezeptor, auf den $\mathrm{D}_{1^{-}}, \mathrm{D}_{3^{-}}$und $\mathrm{D}_{4}$-Rezeptor sowie auf den 5-HT $\mathrm{HA}^{-}$ Rezeptor über partiellen Agonismus wurden verantwortlich gemacht (Kapur und Mamo, 2003).

Theorien der zweiten Gruppe beinhalten, dass einzig die optimale Modulation des $\mathbf{D}_{\mathbf{2}}$-Rezeptors notwendig ist, um die atypische Wirkung zu erzielen (Kapur und Mamo, 2003).

Zur ersten Gruppe von Theorien gehört die sehr bekannte Serotonin-Dopamin-Hypothese. Eine höhere 5 - $\mathrm{HT}_{2}$ - als $\mathrm{D}_{2}$-Rezeptorblockade gilt dabei als ein wesentlicher Faktor für die Atypizität (Meltzer, 1989, 1999). Auch wenn die meisten atypischen Antipsychotika eine höhere Affinität zu 5- $\mathrm{HT}_{2^{-}}$als zu $\mathrm{D}_{2}$-Rezeptoren besitzen, sprechen mehrere Gründe gegen diese Theorie. Nach Kapur und Mamo (2003) gibt es keinen direkten Beweis, dass unter der Therapie mit einem $\mathrm{D}_{2}$-Antagonisten durch Hinzugabe eines 5 - $\mathrm{HT}_{2}$-Antagonisten ein atypisches Profil erlangt wird. Auch scheint der Grad der Atypizität nicht allein durch den Grad des 5- $\mathrm{HT}_{2} / \mathrm{D}_{2}$-Verhältnisses bestimmt zu sein, da einige Antipsychotika mit hohem 5- $\mathrm{HT}_{2} / \mathrm{D}_{2}$-Verhältnis klinisch häufiger zum Auftreten von extrapyramidal-motorischen Wirkungen führen als Antipsychotika mit niedrigerem $5-\mathrm{HT}_{2} / \mathrm{D}_{2}$-Verhältnis. Weiterhin zeigen die meisten Atypika in höheren Dosierungen Eigenschaften, die eher den typischen Antipsychotika entsprechen. Nicht zuletzt zeigen Substanzen, die eine sehr hohe Affinität zum 5- $\mathrm{HT}_{2^{-}}$ Rezeptor allein und kaum Affinität zum $\mathrm{D}_{2}$-Rezeptor besitzen, wie beispielsweise Ritanserin (ein 5- $\mathrm{HT}_{2 \mathrm{~A}} / 5-\mathrm{HT}_{2 \mathrm{C}}$-Antagonist) oder die Substanz MDL 100,907 (ein 5- $\mathrm{HT}_{2 \mathrm{~A}}$-Antagonist), keinerlei antipsychotischen Effekt (Kapur und Mamo, 2003).

Zur zweiten Gruppe von Theorien gehört die Fast-off- $\mathrm{D}_{2}$-Theorie, die besagt, dass die atypischen Antipsychotika eine niedrige Affinität zum $\mathrm{D}_{2}$-Rezeptor besitzen und somit von diesem schnell wieder dissoziieren (Seeman P, 2002). Eine schnellere Dissoziation des Liganden vom Dopamin-Rezeptor bewirkt demnach eine bessere Antwortmöglichkeit innerhalb der phasischen Dopamin-Aktivität und führt somit zu einer geringeren Störung der physiologischen Neurotransmittersignale als bei langsamer Dissoziation (Kapur und Seeman P, 2001). Dies erklärt beispielsweise, warum bei der Therapie mit atypischen Antipsychotika weniger antidopaminerge Nebenwirkungen, wie beispielsweise extrapyramidal-motorische Bewegungsstörungen und Hyperprolaktinämie, auftreten. Auch die Tatsache, dass eine niedrige Dosis eines Atypikums auch 


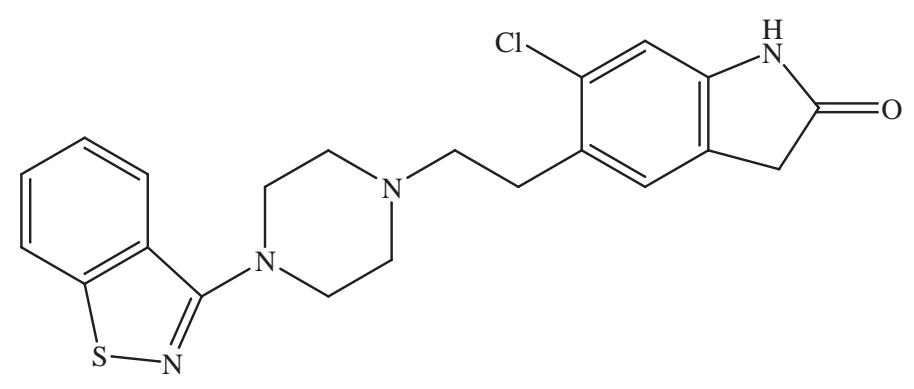

Abbildung 2.1: Struktur von Ziprasidon nach Seeger et al. (1995), S. 102

bei einer L-Dopa-Psychose ${ }^{2}$ wirkt, kann so hergeleitet werden (Seeman P, 2002). Darüber hinaus scheint die Rezeptoraffinität in den unterschiedlichen Hirnregionen eine Rolle zu spielen. Atypische Antipsychotika blockieren bevorzugt die $\mathrm{D}_{2}$-Rezeptoren in extrastriatalen Hirnregionen (besonders im Thalamus bzw. frontalen und temporalen Kortex) und nicht so sehr die $\mathrm{D}_{2}$-Rezeptoren in striatalen Regionen (Kapur und Mamo, 2003).

\subsubsection{Ziprasidon}

$\mathrm{Zu}$ den in Deutschland gebräuchlichsten atypischen Antipsychotika zählen Clozapin (Handelsname z. B. Leponex ${ }^{\circledR}$ ), Olanzapin (Zyprexa ${ }^{\circledR}$ ), Quetiapin (Seroquel ${ }^{\circledR}$ ), Risperidon (Risperdal ${ }^{\circledR}$ ), Ziprasidon (Zeldox ${ }^{\circledR}$ ), Amisulprid (Solian ${ }^{\circledR}$ ), Aripiprazol (Abilify ${ }^{\circledR}$ ) und neuerdings wieder Sertindol (Serdolect ${ }^{\circledR}$ ).

Ziprasidon ist seit 2002 in Deutschland zur Behandlung einer Schizophrenie zugelassen (Pfizer, 2006). Die chemische Bezeichnung für Ziprasidon lautet:

5-\{2-[4-(1,2-Benzisothiazol-3-yl)pipera-zin-1-yl]ethyl\}-6-chlorindolin-2-on

Die Abbildung 2.1 zeigt die Struktur des Moleküls.

Klinische Studien zeigen, dass Ziprasidon gut gegen Positiv- und Negativsymptome sowie gegen affektive Symptome wirkt (Daniel und Copeland, 2000). Die Datenlage unterstützt den Einsatz des Medikaments als ein Mittel der ersten Wahl zur Behandlung der Schizophrenie (Caley und Cooper, 2002). Ziprasidon hat sich sowohl in Kurzals auch in Langzeitstudien gut verträglich gezeigt (Daniel, 2003). Unter Ziprasidon kommt es nur zu einer vernachlässigbaren Gewichtszunahme und in geringem Maße zu Erhöhungen des Glukose- und Lipidspiegels (Daniel, 2003). Darüber hinaus gibt es Hinweise, dass Ziprasidon den atypischen Antipsychotika Olanzapin und Risperidon hinsichtlich der Verbesserung kognitiver Leistungsfähigkeit bei Schizophrenen überlegen ist (Harvey et al., 2004a).

\footnotetext{
${ }^{2}$ Diese bezeichnet eine Psychose, die im Rahmen der Therapie eines Parkinson-Syndroms durch Gabe von L-Dopa, einer hirngängigen Dopaminvorstufe, ausgelöst worden ist.
} 


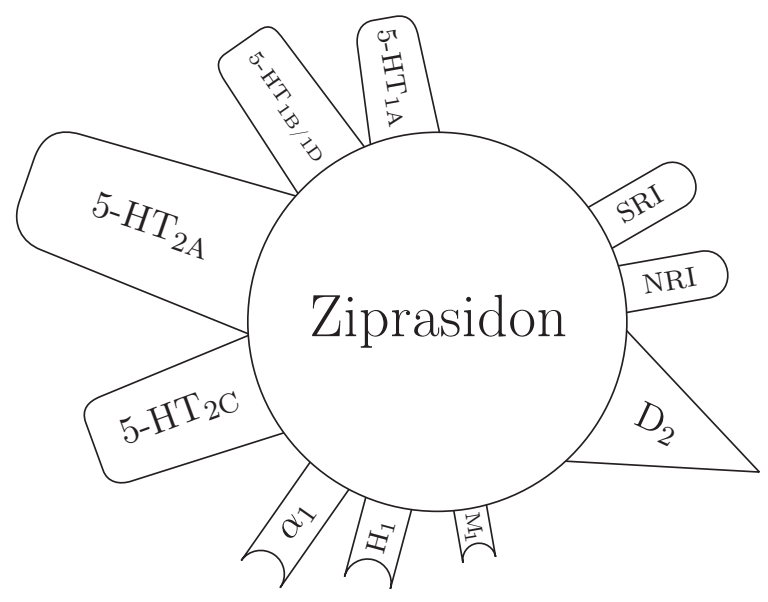

Abbildung 2.2: Rezeptor-Bindungsprofil von Ziprasidon, modifiziert nach Stahl und Shayegan (2003), S. 8

$5-\mathrm{HT}=$ Serotonin-Rezeptoren

$\alpha_{1}=\alpha_{1}$-Adrenozeptor

$\mathrm{H}_{1}=\mathrm{H}_{1}$-Histamin-Rezeptor

$\mathrm{M}_{1}=$ muskarinerger Acetylcholin-Rezeptor vom Typ $\mathrm{M}_{1}$

$\mathrm{D}_{2}=\mathrm{D}_{2}$-Dopamin-Rezeptor

$\mathrm{SRI} / \mathrm{NRI}=$ Serotonin $/$ Noradrenalin Reuptake Inhibitor

Ziprasidon wirkt als kombinierter Agonist und Antagonist an verschiedenen 5-HTRezeptoren. Es ist ein Antagonist am 5- $\mathrm{HT}_{2 \mathrm{~A}^{-}}, 5-\mathrm{HT}_{2 \mathrm{C}^{-}}$und 5- $\mathrm{HT}_{1 \mathrm{~B} / 1 \mathrm{D}^{-} \text {Rezeptor }}$ und ein Agonist am 5-HT 1 A-Rezeptor (Schmidt et al., 2001; Seeger et al., 1995). Ferner besitzt Ziprasidon eine antagonistische Wirkung am $\mathrm{D}_{2}$-Rezeptor und außerdem eine Komponente, die die Wiederaufnahme von Serotonin und Noradrenalin hemmt (Caley und Cooper, 2002; Schmidt et al., 2001; Stahl und Shayegan, 2003; Tatsumi et al., 1999). Eine geringe antagonistische Affinität weist Ziprasidon auch gegenüber dem $\alpha_{1}$-Adrenozeptor, dem $\mathrm{H}_{1}$-Histamin-Rezeptor und in noch geringerem Umfang auch gegenüber dem muskarinergen Acetylcholin-Rezeptor vom Typ $\mathrm{M}_{1}$ auf (Schmidt et al., 2001; Stahl und Shayegan, 2003). Dieses Rezeptor-Bindungsprofil veranschaulicht die Abbildung 2.2.

\subsection{Schlaf}

Entgegen dem äußeren Anschein ist der Schlaf kein passiver Zustand, sondern ein kontrolliert ablaufender, aktiver Prozess im Gehirn. Einen Zugang zur wissenschaftlichtechnischen Erfassung des Schlafs erlangte man erst durch die Möglichkeit der Messung von Hirnströmen mit Hilfe eines Elektroenzephalogramms (EEG) gegen Ende der 20er Jahre des letzten Jahrhunderts. 
In Kombination mit der Aufzeichnung des Muskeltonus durch ein Elektromyogramm (EMG) und der Augenbewegungen mittels Elektrookulogramm (EOG) ergaben sich zusätzliche Informationen. Aserinsky und Kleitman (1953) beschrieben als erste den REM-Schlaf (Rapid eye movement, REM) als ein sich regelmäßig wiederholendes Phänomen, das durch schnelle Augenbewegungen gekennzeichnet ist. Dieses war eine wesentliche Grundlage zur Beschreibung der verschiedenen Schlafstadien.

Während des Schlafs wechseln sich Non-REM-Schlaf (NREM) und REM-Schlaf in charakteristischer Weise ab. Dahinter liegt eine ultradiane ${ }^{3}$ Rhythmik. Man bezeichnet den NREM-Schlaf auch als „orthodoxen Schlaf“. Beim von schnellen Augenbewegungen geprägten REM-Schlaf spricht man von ,paradoxem Schlaf“. Früher bezeichnete man diesen auch als Traumschlaf, weil man davon ausging, dass Träume nur im REM-Schlaf auftreten (Dement und Kleitman, 1957). Diese These gilt jedoch als widerlegt (Foulkes, 1962).

Nach mehreren Jahren uneinheitlicher Methoden veröffentlichten Rechtschaffen und Kales 1968 ein international anerkanntes Manual zur Aufzeichnung und Klassifikation der Schlafstadien, das bis heute Gültigkeit besitzt und die Grundlage der Vergleichbarkeit wissenschaftlicher Studien zum Thema Schlaf bildet.

Nach Rechtschaffen und Kales (1968) unterscheidet man den REM-Schlaf und das Stadium Wach sowie vier NREM-Schlafstadien, im Einzelnen sind dieses Stadium (St.) 1, 2, 3 und 4. Die Stadien 1 und 2 sind durch leichte Erweckbarkeit geprägt. Bei den Stadien 3 und 4 liegt die Weckschwelle wesentlich höher. Die Stadien 1-2 bezeichnet man daher auch als Leichtschlaf, die Stadien 3-4 als Tiefschlaf. Im Englischen wird letzterer als Slow wave sleep (SWS) tituliert, da die Stadien 3-4 im Wesentlichen durch langsame $\delta$-Wellen im EEG charakterisiert werden.

Zur Auswertung wird der Schlaf in jeweils 30 Sekunden andauernde Epochen eingeteilt und jeder Epoche wird ein Schlafstadium zugeordnet. Ist eine Zuordnung nicht möglich, weil z. B. länger als 15 Sekunden andauernde Artefakte auftreten, wird die entsprechende Epoche als Movement time (MT) definiert.

\subsubsection{Schlaf bei gesunden Probanden}

Die folgenden Ausführungen orientieren sich an dem oben genannten Manual von Rechtschaffen und Kales (1968). Ergänzend zu den Kriterien von Rechtschaffen und Kales gibt es mittlerweile spezifizierte Erfassungsregeln für die EEG-Muster. Diese versuchen die Reliabilität der visuellen Auswertung zu erhöhen und können als

\footnotetext{
${ }^{3}$ Eine ultradiane Rhythmik bezeichnet eine Rhythmik, deren Periode im Gegensatz zur zirkadianen (Syn. diurnalen) Rhythmik weniger als 24 Stunden beträgt.
} 
Grundlage für einen standardisierten Algorithmus im Rahmen einer computergestützten Analyse genutzt werden (Rodenbeck et al., 2006).

Vor dem Einschlafen befindet sich ein gesunder Mensch bei geschlossenen Augen im entspannten Wachzustand. Im EEG zeigen sich zu diesem Zeitpunkt vor allem $\alpha$-Wellen und/oder gemischt frequente Wellen mit geringer Spannung. $\alpha$-Wellen besitzen eine Frequenz von $8-12 \mathrm{~Hz}$ und bei den meisten Individuen eine Amplitude von $10-50 \mu \mathrm{V}$ (Rodenbeck et al., 2006).

Das Schlafstadium 1 geht einher mit einer Verlangsamung des EEGs. Es ist weiter gekennzeichnet durch ein EEG von relativ geringer Spannung und gemischter Frequenz, wobei eine Frequenz von $2-7 \mathrm{~Hz}$ vorherrschend ist. Im Verlauf können scharfe Vertexzacken mit höherer Amplitude (bis zu $200 \mu \mathrm{V}$ ) auftreten. Das EOG ist durch langsame rollende Augenbewegungen gekennzeichnet. Der EMG-Tonus ist geringer als beim entspannten Wachsein.

Als nächstes folgt das Stadium 2. Die Grundfrequenz des EEGs ist ähnlich dem Stadium 1. Es kommen jedoch zusätzlich Schlafspindeln und K-Komplexe vor. Schlafspindeln sind in der Amplitude regelmäßig auf- und abschwellende Wellen mit einer Frequenz von 10-14 Hz, ihre Periodenlänge beträgt dabei mindestens $0,5 \mathrm{~s}$. Als KKomplex wird eine negativ-positiv-biphasische Welle mit hoher Amplitude und Dauer von über 0,5 s bezeichnet. Es treten nur zu Beginn dieses Stadiums Augenbewegungen auf und der Muskeltonus nimmt weiter ab.

Das Stadium 3 ist durch weitere Verlangsamung der Wellen im EEG gekennzeichnet. Mit einem Anteil von $20 \%$ bis maximal $50 \%$ kommen jetzt $\delta$-Wellen mit einer Frequenz von $\leq 2 \mathrm{~Hz}$ und einer hohen Amplitude $(>75 \mu \mathrm{V})$ vor. $\delta$-Wellen mit einem Anteil von über $50 \%$ machen das Stadium 4 aus. Während der Stadien 3 und 4 treten keine Augenbewegungen mehr auf und der EMG-Tonus verbleibt auf niedrigem Niveau.

Nach dem Stadium 4 nimmt die Schlaftiefe in entgegengesetzter Richtung wieder ab bis hin zum ersten Auftreten von REM-Schlaf. Dieser kennzeichnet sich durch schnelle Augenbewegungen. Das EEG ähnelt dem Stadium 1 und weist gemischte Frequenzen mit niedriger Amplitude auf. Vertexzacken werden im Gegensatz zu Stadium 1 nicht deutlich. Stattdessen können sägezahnförmig aussehende Wellen auftreten. Der Muskeltonus ist gegenüber anderen Stadien am ausgeprägtesten supprimiert.

Nach Abschluss der ersten REM-Phase ist der erste Schlafzyklus abgeschlossen. Als Schlafzyklus bezeichnet man die Abfolge von Stadium 2, SWS, Stadium 2 und REMSchlaf. Ein solcher Zyklus dauert im Durchschnitt 90 Minuten und kommt in der Nacht bei einem gesunden Menschen etwa vier bis sechs Mal vor. Die einzelnen Zyklen unterscheiden sich durch ihre SWS- und REM-Schlaf-Anteile. Zu Beginn der Nacht sind die SWS-Anteile größer als die REM-Schlaf-Anteile. Gegen Ende der Nachtruhe dominieren die Leichtschlafstadien und der REM-Schlaf. Darüber hinaus nimmt der 


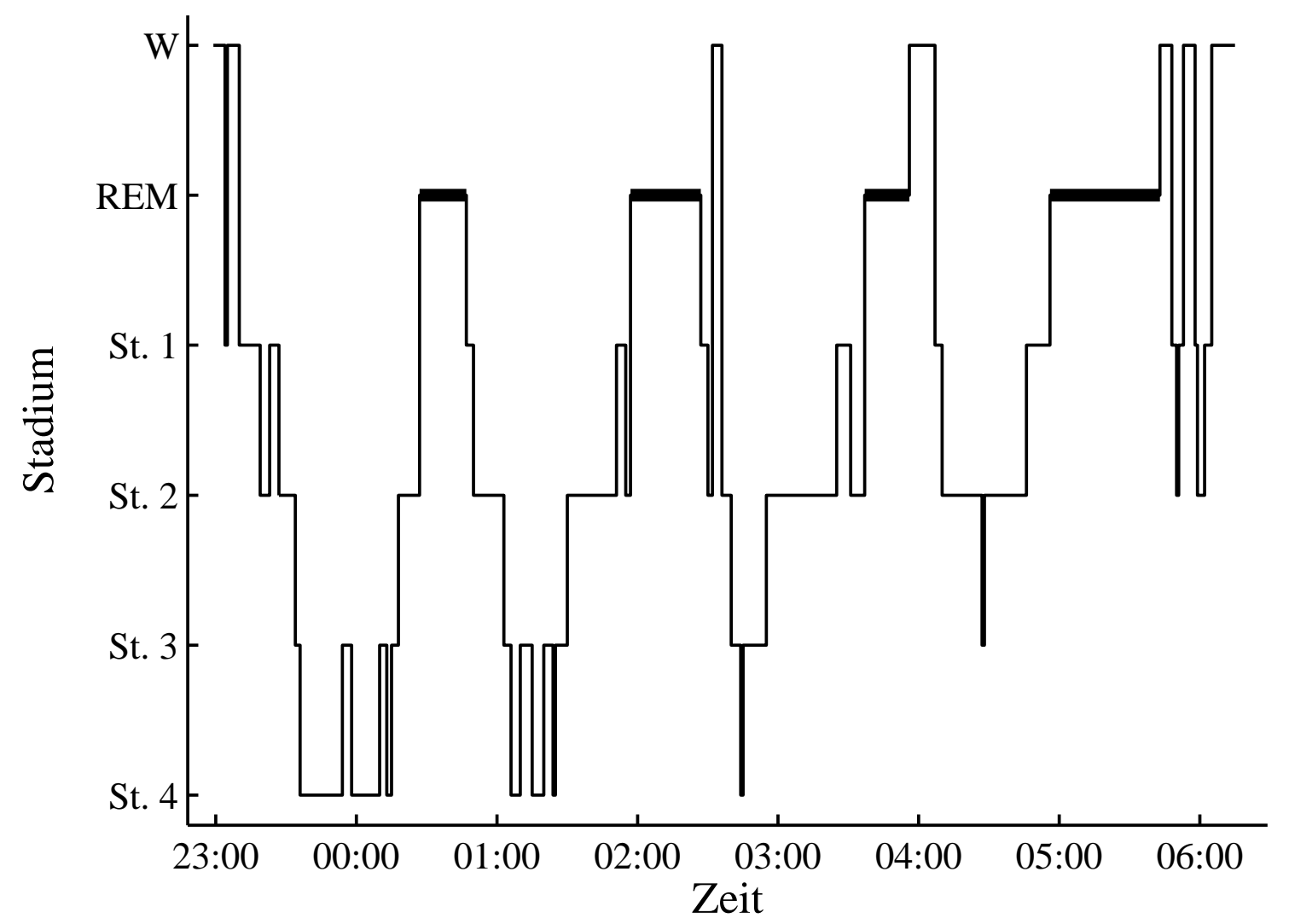

Abbildung 2.3: Schlafstruktur einer jungen gesunden Person nach Riech (2002), S. 29

nächtliche SWS-Anteil mit zunehmendem Lebensalter ab. Die Abbildung 2.3 zeigt die Schlafstruktur (Syn. Schlafarchitektur) einer jungen gesunden Person.

\subsubsection{Funktion und Regulation des Schlafs}

Der Schlaf ist für uns Menschen essenziell notwendig. Experimente zum Schlafentzug an gesunden Probanden zeigen, dass bei entsprechender Dauer der Wachseinsphase das Wachbleiben immer schwieriger wird, Gedächtnis- bzw. Konzentrationsstörungen auftreten und die Leistungsfähigkeit abnimmt, auf äußere Reize adäquat zu reagieren. Schließlich können auch Illusionen ${ }^{4}$ oder sogar Halluzinationen auftreten (Borbély, 1998).

Trotz vieler Forschungsarbeiten ist die Funktion des Schlafs nach wie vor nicht vollständig geklärt (Maquet, 2001). Es gibt mehrere unterschiedliche Erklärungsansätze, die im Folgenden kurz vorgestellt werden sollen. Einige Autoren gehen davon

\footnotetext{
${ }^{4}$ Syn. Verkennung, bezeichnet eine Sinnestäuschung mit gestörter Wahrnehmung realer Objekte.
} 
aus, dass der Schlaf der Energieeinsparung (Berger RJ und Phillips, 1995) dient. Mc Ginty und Szymusiak (1990) weisen auf einen Zusammenhang mit der Thermoregulation hin. Andere halten den Schlaf für einen neuronalen Restaurations- bzw. Entgiftungsprozess (Adam und Oswald, 1977; Inoué et al., 1995). In der allgemeinen Öffentlichkeit ist der Gedanke, dass ein guter Nachtschlaf zu einer besseren Gedächtnisleistung beiträgt, weit verbreitet. In schlafmedizinischen Fachkreisen wurde dies jedoch jahrzehntelang kontrovers diskutiert. Mittlerweile belegen jedoch eine Reihe von Studien, dass der Schlaf der Bewusstseins- und Gedächtnisbildung sowie dem Lernen dient (Frank und Benington, 2006; Hobson und Pace-Schott, 2002; Maquet, 2001).

Zahlreiche Studien zeigen eine herabgesetzte kognitive und psychomotorische Leistungsfähigkeit durch akute Schlafdeprivation ${ }^{5}$ (Dawson und Reid, 1997; Lee HJ et al., 2003). Ebenso führt eine chronische, geringe Reduktion der Schlafzeit, wie sie unter anderem bei Schizophrenen vorkommt, zu kognitiven Leistungseinbußen (Belenky et al., 2003; Van Dongen et al., 2003). Eine Schlaffragmentierung durch kurzzeitiges Erwachen und somit gestörte Schlafkontinuität, wie sie beim SchlafApnoe-Syndrom auftritt, äußert sich neben vermehrter Tagesschläfrigkeit ebenfalls in einer herabgesetzten kognitiven Leistungsfähigkeit (Bonnet, 1985).

Viele Studien haben sich mit der Frage nach der Bedeutung einzelner Schlafstadien für die menschliche Gedächtnisfunktion beschäftigt. In gleicher Weise wie der Schlaf kein homogener Zustand ist, zeigen sich auch das menschliche Gedächtnis und die damit verbundenen Prozesse, zur Erzeugung und Bewahrung von Erinnerungen, als sehr komplex (Walker MP und Stickgold, 2006).

Das Gedächtnis ist beispielsweise keine einzelne Entität, sondern kann in ein deklaratives und ein nicht-deklaratives Gedächtnis geteilt werden. Zusätzlich zu dieser groben Einteilung gibt es noch weitere untergeordnete Teilbereiche, die an dieser Stelle keine Erwähnung finden, jedoch die Komplexität des menschlichen Gedächtnisses unterstreichen. Dem deklarativen Gedächtnis werden bewusst zugängliche Erinnerungen über Tatsachen und Ereignisse zugeordnet (Wissen über das „Was"). Das nichtdeklarative Gedächtnis wird demgegenüber als unbewusst zugänglich betrachtet und enthält prozedurale Erinnerungen (Wissen über das „Wie"), zu denen Tätigkeiten, Gewohnheiten und Fertigkeiten zählen (Walker MP und Stickgold, 2006).

Studien, die sich mit dem Einfluss des Schlafs auf das deklarative bzw. das nichtdeklarative Gedächtnis befasst haben, ergeben teilweise widersprüchliche Ergebnisse. Einige Studien belegen einen Zusammenhang zwischen dem deklarativen Gedächtnis und dem REM-Schlaf (Cipolli et al., 2001; De Koninck et al., 1989), während andere das deklarative Gedächtnis mit SWS-reichen Schlafphasen (Gais und Born, 2004; Plihal und Born, 1997) oder aber auch mit der Länge des NREM-REM-Schlafzyklus

\footnotetext{
${ }^{5}$ Schlafdeprivation bedeutet Schlafentzug durch Verlängerung der Wachseinsphase.
} 
(Smith, 2001) in Verbindung bringen. Nach Walker MP und Stickgold (2006) mögen diese widersprüchlichen Ergebnisse zum Teil methodisch begründet sein, am wahrscheinlichsten erscheint den Autoren jedoch eine Beteiligung sowohl des Tiefschlafs als auch des REM-Schlafs an der Konsolidierung von komplexen, emotional geprägten Erinnerungen.

Das nicht-deklarative Gedächtnis, insbesondere die prozedurale Erinnerung an motorische Fertigkeiten, scheint eine enge Beziehung zum NREM-Schlaf zu besitzen, in einigen Fällen sogar zu bestimmten NREM-Stadien (Walker MP und Stickgold, 2006). So belegen Smith und MacNeill (1994) und Walker MP et al. (2002) eine Korrelation prozeduraler Gedächtnisinhalte mit dem Schlafstadium 2.

Auch bezüglich der Regulation des Schlafs sind noch viele Fragen offen. Nach Borbély und Achermann (2000) gibt es im Wesentlichen drei grundsätzliche Prozesse, denen die Schlafregulation unterliegt. Als erstes ist der Schlaf ein homöostatischer ${ }^{6}$ Prozess, der durch die Mengenverteilung von Schlaf- und Wachzustand beeinflusst wird. Einfach gesagt bedeutet dies, je länger die Wachdauer besteht, desto größer ist der Schlafdruck. Zweitens spielt ein zirkadianer Prozess eine Rolle; zu einer bestimmten Tageszeit ist die Schlafneigung größer oder kleiner, unabhängig von vorherigem Schlaf oder Wachsein. Drittens ist ein ultradianer Rhythmus von Bedeutung, der durch das alternierende Auftreten von REM- und NREM-Schlaf repräsentiert wird. Wie diese Prozesse miteinander zusammenhängen und gesteuert werden, ist Gegenstand zahlreicher Forschungsarbeiten.

Es existieren zwei grundlegende Modelle, die die Regulation des Schlafs zu erklären versuchen: das ,reziproke Interaktionsmodell“ nach Hobson et al. (1975) und das „Zwei-Prozess-Modell“ nach Borbély (1982).

Das reziproke Interaktionsmodell nach Hobson et al. (1975) schreibt einem Netzwerk cholinerger und aminerger Neurone im Hirnstamm schlafregulatorische Funktionen zu. Diese Neurone wirken auf die ultradiane Regulation von REM- und NREMSchlaf. Cholinerge Neurone (ACh) in der Formatio reticularis des Hirnstamms (FR) haben dem Modell zufolge eine REM-Schlaf erzeugende Funktion. Ihre Gegenspieler sind die noradrenergen (NA) und serotonergen (5-HT) Neurone, die zusammen als aminerge Neurone bezeichnet werden. Die noradrenergen Neurone sind vorwiegend im Locus coeruleus (LC) und die serotonergen in den dorsalen Raphé-Kernen (RK) lokalisiert. Die dazugehörigen Botenstoffe sind Serotonin (5-HT) und Noradrenalin (NA) bzw. für die cholinergen Neurone Acetylcholin (ACh). Eine reziproke Interaktion besteht zwischen den Neuronengruppen. Während cholinerge Neuronen die aminergen Neurone aktivieren, hemmen die aminergen im Gegenzug die cholinergen Neurone. Neue wissenschaftliche Erkenntnisse können zum Teil in das ursprüngliche

\footnotetext{
${ }^{6}$ Homöostase bezeichnet die Fähigkeit eines Systems, sich durch Rückkopplung selbst innerhalb gewisser Grenzen in einem stabilen Zustand zu halten.
} 


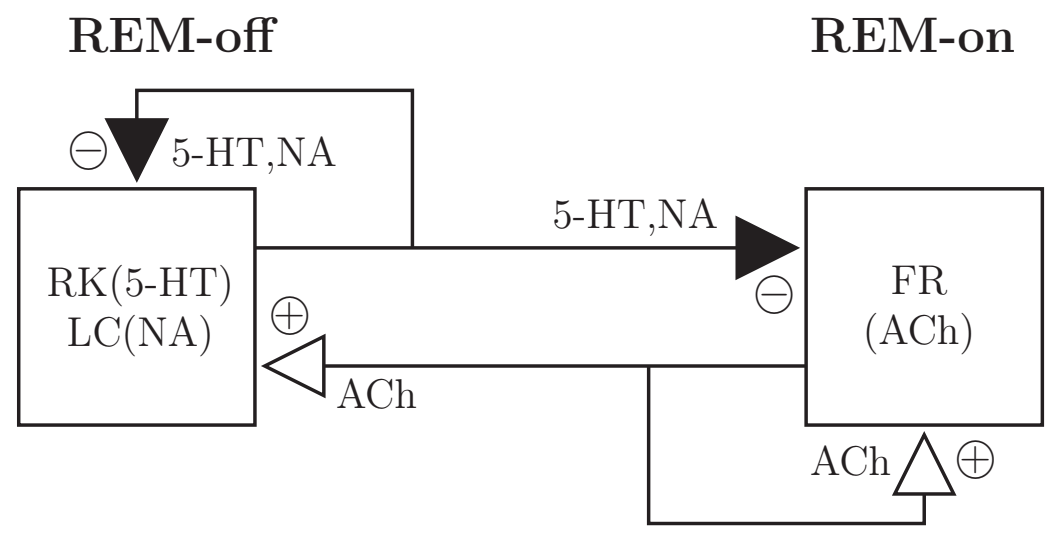

Abbildung 2.4: Das reziproke Interaktionsmodell, modifiziert nach Pace-Schott und Hobson (2002), S. 596

$$
\begin{aligned}
5-\mathrm{HT} & =\text { Serotonin } \\
\mathrm{NA} & =\text { Noradrenalin }, \\
\mathrm{ACh} & =\text { Acetylcholin } \\
\mathrm{RK} & =\text { Raphé-Kerne } \\
\mathrm{LC} & =\text { Locus coeruleus } \\
\mathrm{FR} & =\text { Formatio reticularis }
\end{aligned}
$$

Modell integriert werden, auf diese soll aber an dieser Stelle nicht eingegangen werden (Hobson und Pace-Schott, 2002; Pace-Schott und Hobson, 2002). Abbildung 2.4 veranschaulicht das ursprüngliche reziproke Interaktionsmodell.

Das Zwei-Prozess-Modell nach Borbély (1982) erklärt die Schlafregulation durch zwei ineinander greifende Prozesse S und C. Der Prozess S ist ein homöostatischer schlafinduzierender Prozess, der mit zunehmender Wachdauer ansteigt und durch Schlaf reduziert werden kann. Im Gegensatz dazu spiegelt der zirkadiane Prozess C einen Schlafdrang wider, der von der Uhrzeit abhängt und oszillatorisch schwankt. Ein hoher Anteil des Prozesses S liegt, der Hypothese nach, zu Beginn der Nacht vor und unterdrückt die REM-Aktivität. Abbildung 2.5 zeigt ein Schema des ZweiProzess-Modells.

\subsubsection{Schlaf bei depressiven Patienten}

Mehr als $90 \%$ der depressiven Patienten berichten von verminderter Schlafqualität (Riemann et al., 2001). Sie leiden vor allem unter Einschlafschwierigkeiten, häufigen Aufwachvorgängen und unter Früherwachen, was sich auch im polysomnographisch erfassten Schlaf widerspiegelt. Einige Studien weisen sogar darauf hin, dass Schlafstörungen einen Risikofaktor für die spätere Entwicklung einer Depression darstellen (Chang et al., 1997; Riemann et al., 2001). 


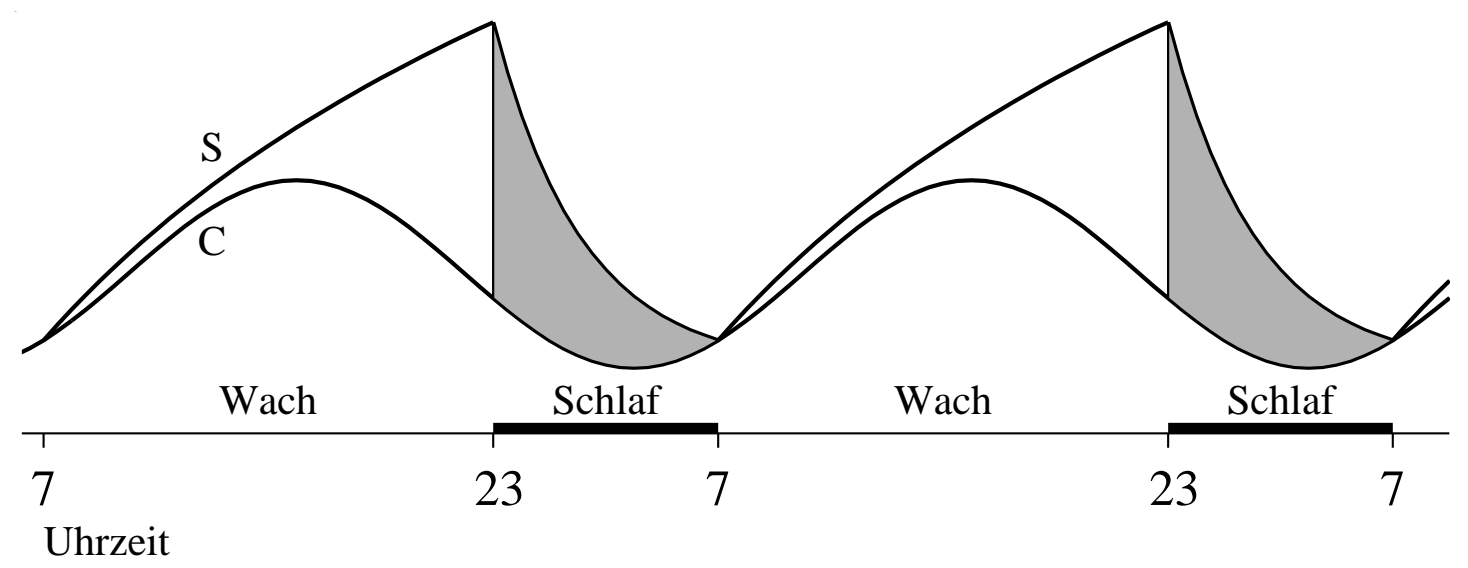

Abbildung 2.5: Das Zwei-Prozess-Modell, Abbildung aus Borbély (1998) $\mathrm{S}=$ homöostatischer Prozess $\mathrm{S}$, $\mathrm{C}=$ zirkadianer Prozess $\mathrm{C}$

Polysomnographisch ist der Schlaf Depressiver durch eine verminderte Schlafkontinuität geprägt. Dies drückt sich in einer verkürzten Gesamtschlafzeit, einer reduzierten Schlafeffizienz, einer verlängerten Einschlaflatenz, einer längeren Wachzeit in den frühen Morgenstunden und zahlreichen Aufwachvorgängen aus (Benca, 2000; Benca et al., 1992). Zudem zeigt sich eine Verminderung des Tiefschlaf-Anteils (Benca, 2000). Ferner besteht eine Disinhibition des REM-Schlafs in Form einer verkürzten REM-Latenz, eines erhöhten REM-Anteils (besonders im ersten Schlafzyklus) und einer erhöhten REM-Dichte (Benca, 2000; Riemann et al., 2001). In Abbildung 2.6 ist beispielhaft die Schlafstruktur eines Depressiven abgebildet.

In den polysomnographischen Studien zur Schlafstruktur depressiver Patienten zeigt sich ein profundes und weitgehend homogenes Bild. Einschränkend muss aber beachtet werden, dass sich viel mehr Studien mit affektiven Störungen als mit anderen psychiatrischen Erkrankungen (wie beispielsweise Schizophrenie, Angsterkrankungen, Alkoholismus, Borderline-Persönlichkeitsstörungen oder Demenz) beschäftigt haben, was statistische Vergleiche erschwert (Benca et al., 1992). Gegenüber anderen psychiatrischen Erkrankungen lässt sich die Depression bisher weder durch spezifisch veränderte Schlafparameter noch durch deren Kombination signifikant abgrenzen (Benca et al., 1992).

\subsubsection{Schlaf bei schizophrenen Patienten}

Schlafstörungen kommen bei Schizophrenen sehr häufig vor, werden jedoch meist nicht als dominierende Beschwerde vorgetragen. Sie bedeuten aber für schizophrene Patienten eine deutliche Einschränkung der Lebensqualität wie Ritsner et al. (2004) 


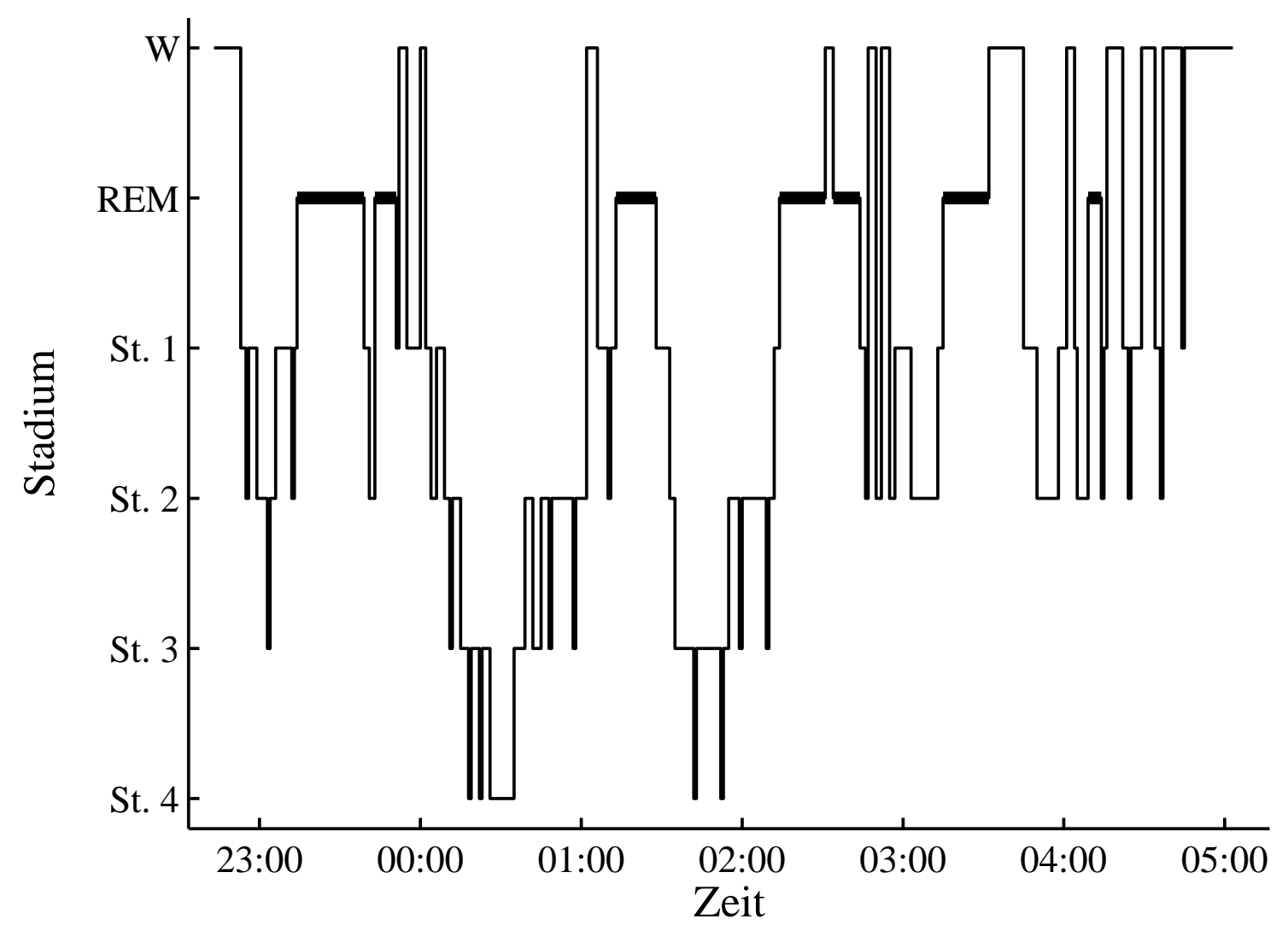

Abbildung 2.6: Schlafstruktur eines Depressiven nach Riech (2002), S. 32

belegen. Klinisch treten insomnische Beschwerden zudem oft als ein Prodromalsymptom im Rahmen eines Rückfalls auf (Benson und Zarcone, 2000; Van Kammen et al., 1986).

In zahlreichen polysomnographischen Studien ist der Schlaf schizophrener Patienten untersucht worden. Die Vergleichbarkeit der methodisch sehr unterschiedlich angelegten Studien ist allerdings nicht immer gegeben (Monti JM und Monti D, 2004, 2005).

Die Mehrzahl der Studien belegt Einschlaf- und Durchschlafstörungen (Monti JM und Monti D, 2005). Die Schlafkontinuität dieser Patienten ist deutlich herabgesetzt, was sich vor allem in einer verminderten Gesamtschlafzeit, einer herabgesetzten Schlafeffizienz, einer erhöhten Schlaffragmentierung mit häufigen Aufwachvorgängen und einer verlängerten Einschlafzeit ausdrückt (Caldwell und Domino, 1967; Keshavan et al., 1998; Lauer et al., 1997; Monti JM und Monti D, 2005; Zarcone et al., 1987).

Es zeigt sich auch eine Verminderung des Tiefschlaf-Anteils (Caldwell und Domino, 1967; Keshavan et al., 1998; Lauer et al., 1997; Monti JM und Monti D, 2005; Zarcone 
et al., 1987). Hier liegt allerdings eine höhere Variabilität zwischen den Studienergebnissen vor.

Mehrere Studien belegen eine im Vergleich zu Gesunden verkürzte REM-Latenz (Hudson et al., 1993; Tandon et al., 1992; Zarcone et al., 1987), während der REMAnteil meist unverändert bleibt (Monti JM und Monti D, 2005).

Die Veränderungen der REM-Dichte werden kontrovers diskutiert. In einigen Studien wird eine Erhöhung der REM-Dichte dokumentiert (Gulevich et al., 1967; Hudson et al., 1993), während andere Studien keine Differenz im Vergleich zu gesunden Kontrollpersonen feststellen (Benson und Zarcone, 1993; Feinberg et al., 1965).

\subsubsection{Hypothesen zum Schlaf bei Depression und Schizophrenie}

Inzwischen existieren vier größere Erklärungsansätze für den charakteristisch veränderten Schlaf bei Depression, auf die an dieser Stelle kurz eingegangen werden soll (Benca, 2000).

Die REM-Druck-Hypothese wurde vor allem in den 1970er Jahren postuliert (Vogel, 1975; Vogel et al., 1975). Sie besagt, dass die Depression durch große Mengen an REM-Schlaf und durch daraus resultierenden, erniedrigten REM-Druck verursacht wird. Die REM-Druck-Hypothese wird vor allem durch Studien zur antidepressiven Wirkung von Schlafentzug unterstützt. Eine Schlafdeprivation wirkt demzufolge antidepressiv, da sie den REM-Schlaf unterdrückt und damit den REM-Druck erhöht. Unterstützt wird die Hypothese durch die Tatsache, dass die meisten Antidepressiva den REM-Schlaf unterdrücken (Gursky und Krahn, 2000; Mayers und Baldwin, 2005; Riemann et al., 2001; Rijnbeek et al., 2003; Sharpley und Cowen, 1995; Winokur et al., 2001). Früher postulierte man sogar einen Zusammenhang zwischen Unterdrückung des REM-Schlafs durch Antidepressiva und antidepressivem Effekt (Kupfer, 1976; Kupfer et al., 1976). Vor dem Hintergrund, dass es auch Medikamente, wie etwa Bupropion oder Nefazodon, gibt, die eine effektive antidepressive Medikation darstellen, den REM-Schlaf aber nicht unterdrücken, gilt dies inzwischen als widerlegt (Benca, 2000).

Eine weitere Hypothese ist die Cholinerg-aminerge-Imbalance. Diese bezieht sich auf das in Abschnitt 2.2.2 vorgestellte reziproke Interaktionsmodell zur Schlafregulation von Hobson et al. (1975). Dieses Modell besagt, dass der REM-Schlaf durch cholinerge Neurone getriggert und durch aminerge Neurone gehemmt wird. Der vermehrte REM-Schlaf bei der Depression kann somit entweder über eine cholinerge Hyperaktivität oder durch eine aminerge Hypoaktivität hervorgerufen werden. Zahlreiche Studien belegen eine cholinerge Hyperaktivität bei Depressiven. Beispielsweise 
zeigt sich eine deutlich herabgesetzte REM-Latenz im Vergleich zu gesunden Kontrollen nach Gabe der muskarinergen Acetylcholin-Rezeptor-Agonisten Arecolin oder RS-86 (Berger M et al., 1989; Gillin et al., 1991).

Die Phase-Advance-Theorie besagt, dass ein zirkadianer Oszillator bei der Depression eine vorgezogene Phase aufweist. Dies bedeutet, dass die NREM-REMSchlafzyklen eines Depressiven, der um 24 Uhr zu Bett geht, den NREM-REM-Zyklen eines Gesunden gegen sechs Uhr morgens gleichen (Benca, 2000). Unterstützt wird diese Hypothese durch den antidepressiven Effekt einer Vorverlegung der Schlafphase (Wehr et al., 1979). Nicht alle Studien konnten jedoch den antidepressiven Effekt einer vorgezogenen Schlafphase bestätigen (Benca, 2000).

Die Prozess-S-Defizienz-Hypothese knüpft an das in Abschnitt 2.2.2 beschriebene Zwei-Prozess-Modell zur Schlafregulation von Borbély (1982) an. In Anlehnung an das Modell unterdrückt ein Überwiegen des Prozesses S den REM-Schlaf. Nach Borbély und Wirz-Justice (1982) ist der Prozess S bei der Depression defizient. Einiges spricht auch für das Vorliegen einer Cholinerg-aminergen-Imbalance bei der Schizophrenie (Benson und Zarcone, 2000), allerdings nicht in so ausgeprägtem Maße wie bei der Depression. Die Imbalance-Theorie bezieht sich auf das reziproke Interaktionsmodell zur Schlafregulation von Hobson et al. (1975) (vgl. Abschnitt 2.2.2). Dieses besagt, dass der REM-Schlaf durch cholinerge Neurone getriggert und durch aminerge Neurone gehemmt wird.

Basierend auf der Vorstellung einer Überaktivität dopaminerger Strukturen im Rahmen einer Schizophrenie sind Studien durchgeführt worden, die die Effekte von Dopamin-Agonisten und Dopamin-Antagonisten auf den Schlaf untersuchen.

Die $\mathrm{D}_{2}$-Rezeptor-Agonisten Apomorphin, Bromocriptin und Pergolid verstärken die Wachheit. YM-09151-2, ein substituiertes Benzamid, das als selektiver $\mathrm{D}_{2}$-RezeptorAntagonist wirkt, verstärkt dagegen den (Leicht-)Schlaf (Monti JM und Monti D, 2005).

Es liegt jedoch nahe, dass die Schlafstörungen schizophrener Patienten nicht allein dopaminabhängig sind, sondern dass auch andere Neurotransmittersysteme (wie beispielsweise GABA) involviert sind (Monti JM und Monti D, 2005). Es gibt zudem Hinweise, dass eine cholinerge Hyperaktivität ähnlich wie bei der Depression eine Rolle spielt (Benson und Zarcone, 2000). 


\subsubsection{Zusammenhang zwischen Schlaf und Symptomatik bei Schizophrenie}

Wie dargelegt, weist der Schlaf schizophrener Patienten einige charakteristische Züge auf. Einige Schlafforscher haben daher versucht, den Zusammenhang zwischen gestörten Schlafparametern und der klinischen Symptomatik zu eruieren.

Einige Studien zeigen, dass der Schweregrad der Schizophrenie mit einer erhöhten Wachzeit, mit reduziertem Tiefschlaf- und REM-Anteil sowie mit einer verkürzten REM-Latenz (Benson und Zarcone, 2000; Kempenaers et al., 1988; Tandon et al., 1992) korreliert.

Die Positivsymptomatik steht in Zusammenhang mit einer verkürzten REM-Latenz (Lauer et al., 1997; Tandon et al., 1992), erhöhter REM-Dichte (Benson und Zarcone, 1993; Feinberg et al., 1965), verminderter Schlafeffizienz (Neylan et al., 1992) und verlängerter Schlaflatenz (Zarcone und Benson, 1997).

Das Ausmaß der Negativsymptomatik korreliert mit reduziertem Tiefschlaf-Anteil (Ganguli et al., 1987; Keshavan et al., 1995a) und verkürzter REM-Latenz (Tandon et al., 1992; Taylor et al., 1991).

Darüber hinaus haben Wissenschaftler in longitudinalen Studien eine Assoziation des Tiefschlaf-Defizits (Keshavan et al., 1995b; Tandon et al., 2000) sowie der verkürzten REM-Latenz (Goldman et al., 1996; Taylor et al., 1991) mit einem schlechteren Krankheitsverlauf aufzeigen können.

\subsection{Wirkung der Antipsychotika auf den Schlaf}

Die Aussagekraft älterer Studien zur Frage nach der Wirkung von Antipsychotika auf den Schlaf ist begrenzt. Insbesondere Studien, die sich mit der Wirkung der klassischen Antipsychotika beschäftigt haben, sind nicht plazebokontrolliert und doppelblind durchgeführt worden (Freemon, 1973). Zudem sind die frühen Arbeiten noch nicht nach den internationalen Richtlinien von Rechtschaffen und Kales (1968) durchgeführt worden. Bei Studien an Schizophrenen kommt hinzu, dass die Patienten beispielsweise nicht ausreichend in krankheitsspezifische Subtypen (paranoid, kataton, undifferenziert) oder Krankheitsphasen (akutes, subakutes, subchronisches bzw. chronisches Stadium) eingeteilt worden sind. Die Vormedikation ist häufig auch nicht hinreichend beachtet worden und/oder nur eine sehr kleine Anzahl von Patienten ist in die Studien eingeschlossen worden (Lauer et al., 1997; Monti JM und Monti D, 2004, 2005). Ferner ist die Anzahl der Studien, die die Auswirkungen atypischer Antipsychotika untersuchen, sehr begrenzt. 


\subsubsection{Wirkung klassischer Antipsychotika bei schizophrenen Patienten}

In einer Studie zur Wirkung klassischer Antipsychotika bei schizophrenen Patienten bewirkt die Gabe von $50 \mathrm{mg}$ Promethazin eine Zunahme der Gesamtschlafzeit und eine Abnahme des REM-Anteils, während unter $5 \mathrm{mg}$ Trifluoperazin ein tendenziell höherer REM-Anteil bei unveränderter Gesamtschlafzeit auftritt (Brannen und Jewett, 1969).

Eine dreiwöchige Therapie mit $400 \mathrm{mg}$ Chlorpromazin führt bei Schizophrenen zu einer Verbesserung der Schlafkontinuität und zu einer leichten Zunahme der REMLatenz, der REM-Dichte und des Tiefschlafs (Kaplan et al., 1974).

Unter Pimozid zeigt sich nach etwa einmonatiger Gabe bei einer gemischten Patientengruppe eine Zunahme der Schlafkontinuität und des Stadiums 2 (Gillin et al., 1977).

Keshavan et al. (1996) untersuchen den Schlaf schizophrener Patienten unter dem Einfluss verschiedener, zumeist klassischer Antipsychotika (vor allem Haloperidol). Nach vierwöchiger Therapie zeigt sich eine Verbesserung der Schlafkontinuität und nur eine geringfügige Erhöhung der REM-Latenz. Nach Ablauf eines Jahres sind die REM-Latenz, der REM-Anteil und die REM-Dichte erhöht, während andere Schlafparameter einschließlich des Tiefschlafs unbeeinflusst geblieben sind.

Maixner et al. (1998) studieren den Schlaf Schizophrener unter Haloperidol und Thiothixen. Nach drei- bis vierwöchiger Gabe nehmen die Schlafkontinuitätsparameter, die REM-Latenz und tendenziell auch der Tiefschlaf-Anteil zu.

Im Vergleich zu behandlungsnaiven schizophrenen Patienten zeigen Schizophrene unter der Therapie mit Haloperidol oder Flupentixol eine Zunahme der Schlafkontinuität und REM-Dichte (Wetter et al., 1996).

Einige Studien untersuchen ferner den Schlaf nach plötzlicher Beendigung einer kontinuierlichen Therapie mit Haloperidol im Hinblick auf die damit verbundenen Absetzphänomene. Thaker et al. (1989) stellen dabei eine Verminderung der Gesamtschlafzeit und des REM-Schlafs fest. Eine andere Arbeitsgruppe teilt ihre Patienten in eine nach dem Absetzen der Haloperidol-Medikation klinisch stabil gebliebene Gruppe und eine Gruppe mit Krankheitsrückfall ein. In der Gruppe der erneut erkrankten Patienten zeigt sich eine Verminderung der Gesamtschlafzeit, der Schlafeffizienz und des Stadiums 2 (Neylan et al., 1992). Nofzinger et al. (1993) untersuchen den Schlaf klinisch stabiler, schizophrener Patienten unter Haloperidol sowie jeweils zwei und sechs Wochen nach dem Absetzen Haloperidols. Nach zwei Wochen zeigt sich dabei eine Erhöhung des Stadiums 2 und eine Verminderung des Stadiums 4. Nach sechs 
Wochen zeigt sich zudem eine signifikante Verminderung der Gesamtschlafzeit, der REM-Latenz und der REM-Dichte bei gleich bleibendem REM-Anteil.

In der Gesamtheit der Studien wird deutlich, dass eine Behandlung schizophrener Patienten mit klassischen Antipsychotika in den meisten Fällen zu einer Zunahme der Schlafkontinuität und damit zu einer Verbesserung des Schlafs führt. Relativ konstant nimmt zudem die REM-Latenz zu. Die Wirkung auf weitere Schlafparameter ist uneinheitlich. Ein Absetzen der Medikation führt vorwiegend zu einer Störung der Schlafkontinuität und zu einer Reduktion der REM-Latenz, was einem gegenteiligen Medikationseffekt gleichkommt.

\subsubsection{Wirkung atypischer Antipsychotika bei schizophrenen Patienten}

In einer Studie an fünf Patienten zur Wirkung atypischer Antipsychotika bei schizophrenen Patienten führt die Gabe von 250-500 mg Clozapin zu einer deutlichen Erhöhung des REM-Anteils, insbesondere bei begleitend auftretender Körpertemperaturerhöhung (Blum und Girke, 1973). Weitere Schlafparameter sind nicht berichtet worden.

Rüther et al. (1976) stellen nach 14-tägiger Behandlung mit 200-400 mg Clozapin bei einer kleinen Patientengruppe ebenfalls eine Erhöhung des REM-Schlafs fest. Darüber hinaus dokumentieren die Autoren eine Reduktion des Tiefschlafs. In späteren Studien an größeren Patientengruppen kann die Erhöhung des REM-Schlafs nicht mehr reproduziert werden, stattdessen bleibt in diesen Studien der REM-Anteil unter Clozapin weitgehend unverändert (Hinze-Selch et al., 1997; Lee JH et al., 2001; Wetter et al., 1996). In den Studien von Hinze-Selch et al. (1997) und Wetter et al. (1996) wird allerdings eine Erhöhung der REM-Dichte beschrieben. Lee JH et al. (2001) dokumentieren diesen Parameter nicht. Die von Rüther et al. (1976) berichtete Verminderung des Tiefschlafs kann nur in der Studie von Hinze-Selch et al. (1997) bestätigt werden, während Lee JH et al. (2001) und Wetter et al. (1996) keine signifikante Veränderung des Tiefschlafs feststellen können. Die Schlaflatenz und die REM-Latenz zeigen sich in zwei Studien nicht signifikant verändert (Hinze-Selch et al., 1997; Lee JH et al., 2001). Wetter et al. (1996) dagegen berichten über eine verminderte Schlaflatenz bei statistisch unveränderter REM-Latenz.

Einheitlich zeigen die neueren Studien eine Erhöhung der Schlafkontinuität und des Stadiums 2 durch Clozapin (Hinze-Selch et al., 1997; Lee JH et al., 2001; Wetter et al., 1996).

Auch nach ein- bis zweitägiger Gabe von 10 mg Olanzapin kommt es bei Schizophrenen zu einer Erhöhung der Schlafkontinuität, des Stadiums 2 und des Tiefschlafs 
sowie zu einer Verkürzung des Stadiums 1 (Salin-Pascual et al., 1999). Darüber hinaus zeigt sich eine Erhöhung der REM-Dichte bei gleichbleibender REM-Latenz und unverändertem REM-Anteil.

Eine längerfristige Applikation von 15-20 mg Olanzapin für vier Wochen zeigt im Wesentlichen analoge Ergebnisse. Auch hier kommt es zu einer Zunahme der Schlafkontinuität, der REM-Dichte und des Tiefschlafs (Müller MJ et al., 2004). Die im REM-Schlaf verbrachte Zeit nimmt allerdings in dieser Studie zu.

Die Wirkung Risperidons auf den Schlaf von schizophrenen Patienten ist nur in einer einzigen Parallelgruppen-Studie untersucht worden (Yamashita et al., 2002). Diese Studie ist eingeschränkt aussagekräftig, da kein Plazebo-Vergleich durchgeführt wurde. Stattdessen wurde die Risperidon-Gruppe direkt mit einer Gruppe unter Haloperidol verglichen. Die Autoren stellen eine Erhöhung des Tiefschlafs bei sonst unverändertem Schlafmuster fest.

Weitere atypische Antipsychotika, wie Amisulprid, Aripiprazol, Quetiapin, Sertindol und Ziprasidon, sind bisher nicht bezüglich ihres Effekts auf den polysomnographisch erfassten Schlaf bei Schizophrenen untersucht worden.

Zusammengefasst zeigt sich unter Clozapin und Olanzapin eine Verbesserung der Schlafkontinuität mit Zunahme von Gesamtschlafzeit und Schlafeffizienz. Sofern in den Studien erfasst, ist auch die REM-Dichte erhöht. Uneinheitliche Effekte zeigen sich bezüglich der im REM-Schlaf und im Tiefschlaf verbrachten Zeit. Die Tabelle 5.1 in der Diskussion zeigt eine Übersicht der Wirkung atypischer Antipsychotika bei schizophrenen Patienten (und bei Gesunden).

\subsubsection{Wirkung klassischer Antipsychotika bei gesunden Probanden}

In Studien zur Wirkung klassischer Antipsychotika bei gesunden Probanden zeigt sich unter $100 \mathrm{mg}$ Chlorpromazin ein unveränderter REM-Anteil bei verkürzter REMLatenz. Auch eine Zunahme des Tiefschlafs wird berichtet (Lester und GuerreroFigueroa, 1966).

Eine andere Studie stellt Unterschiedliches zum REM-Schlaf fest. Hier führen $100 \mathrm{mg}$ Chlorpromazin zu einer Verminderung des REM-Schlafs, während es nach $25 \mathrm{mg}$ zu einem deutlichen Anstieg des REM-Anteils kommt (Lewis und Evans, 1969). Lewis und Evans (1969) können ferner nur nach $25 \mathrm{mg}$ Chlorpromazin eine Zunahme der Gesamtschlafzeit feststellen. Nach 100 mg zeigen sich keine signifikanten Veränderungen bezüglich der Gesamtschlafzeit und der REM-Latenz. 
$150 \mathrm{mg}$ Chlorpromazin führen nach Lester et al. (1971) zu einer Verkürzung der REM-Latenz und tendenziell zu erhöhtem Tiefschlaf.

Eine längere Einnahme von $100 \mathrm{mg}$ Chlorpromazin über vier Wochen resultiert vor allem in einer Verlängerung der Gesamtschlafzeit bei weitgehend unbeeinflusstem Tiefschlaf- und REM-Anteil sowie gleichbleibender REM-Latenz (Hartmann und Cravens, 1973).

Die wie Chlorpromazin zur Gruppe der Phenothiazine zählenden Medikamente Promethazin und Mesoridazin beeinflussen den Schlaf unterschiedlich. Während $10 \mathrm{mg}$ Mesoridazin zu einer Zunahme der im REM-Schlaf verbrachten Zeit führen (Adam et al., 1976), zeigt sich unter $50 \mathrm{mg}, 100 \mathrm{mg}$ und $200 \mathrm{mg}$ Promethazin eine Reduktion des REM-Schlafs mit Verlängerung der REM-Latenz bei weitgehend unveränderter REM-Dichte (Risberg et al., 1975). Darüber hinaus beobachten die Autoren bei $50 \mathrm{mg}$ und $100 \mathrm{mg}$ Promethazin einen Anstieg des Stadiums 2 und bei $100 \mathrm{mg}$ einen Anstieg der im Tiefschlaf verbrachten Zeit (Risberg et al., 1975).

1,5 mg Haloperidol lassen den REM-Anteil unbeeinflusst. Auf weitere Schlafparameter geht die Studie, die dies ermittelt hat, nicht ein (Nakazawa et al., 1977).

Auch Pinto et al. (2002) zeigen unter $5 \mathrm{mg}$ Haloperidol eine gleich bleibende im REM-Schlaf verbrachte Zeit, zudem aber auch eine nicht signifikant veränderte REMDichte. Weitere Schlafparameter werden nicht berichtet.

Eine weitere Studie untersucht lediglich die Wirkung von 3,5 mg Haloperidol, intravenös appliziert, auf einen kurzen Tagesschlaf (Clarenbach et al., 1978). In dieser Studie zeigt sich eine im Vergleich zu Plazebo verkürzte Schlaflatenz und ein verminderter Anteil an Stadium 2 sowie ein größerer wach verbrachter Anteil (Clarenbach et al., 1978).

Nach Gabe von $1 \mathrm{mg}$ bzw. $4 \mathrm{mg}$ Pimozid zeigen sich nur minimale Veränderungen des Schlafmusters. Lediglich eine Verlängerung der ersten REM-Phase nach $1 \mathrm{mg}$ ist statistisch signifikant (Sagalés und Erill, 1975).

2 mg Pimozid über 5 Tage gegeben führen zu einer Abnahme der Wachzeit während der Schlafphase (Puca et al., 1978).

Die einzige Studie zu Sulpirid ( $5 \mathrm{mg} / \mathrm{kg}$ Körpergewicht) ist im Rahmen einer Untersuchung zu Trauminhalten durchgeführt worden. Durch nächtliches Aufwecken ist die Aussagekraft dieser Studie eingeschränkt. Dennoch zeigt sich nach Sulpirid eine höhere Schlafeffizienz unter Abnahme des Stadiums Wach bei gleichbleibender Gesamtschlafzeit. Ferner verkürzt sich unter Medikation das Stadium 1 und es werden weniger Körperbewegungen während des Schlafs registriert (Gaillard und Moneme, 1977). 
Die Datenlage zur Wirkung klassischer Antipsychotika auf den Schlaf Gesunder lässt aufgrund der wenigen und eingeschränkt interpretierbaren Studien keine einheitliche Aussage zu. Es lassen sich keine substanzübergreifenden Effekte auf Schlafkontinuität, einzelne Schlafstadien oder Latenzen ableiten. Es ist daher nahe liegend, dass die bei schizophrenen Patienten beobachteten klaren Veränderungen des Schlafs unter klassischen Antipsychotika nicht nur primäre Medikamenteneffekte darstellen, sondern vielmehr auch als Folge einer gebesserten Symptomatik angesehen werden können.

\subsubsection{Wirkung atypischer Antipsychotika bei gesunden Probanden}

Die Gabe von $25 \mathrm{mg}$ Clozapin für drei Tage führt in Studien zur Wirkung atypischer Antipsychotika bei gesunden Probanden zu einer Zunahme der Gesamtschlafzeit und zu einer Abnahme des Tiefschlafs, insbesondere des Stadiums 4. Ferner zeigt sich eine Erhöhung der REM-Dichte bei sonst statistisch unveränderten REM-Parametern. Auch die Abnahme der Bewegungen im Schlaf ist statistisch signifikant (Touyz et al., 1977).

Touyz et al. (1978) dokumentieren in einer späteren Studie nach Gabe einer niedrigeren Clozapin-Dosis (12,5 mg) über knapp zwei Wochen eine Reduktion des Stadiums 1. Im Gegensatz zur früheren Studie zeigt sich die REM-Dichte unverändert bei aber sonst tendenziell vermindertem REM-Anteil. Ebenfalls kann entgegen der Studie von 1977 keine statistisch signifikante Veränderung von Gesamtschlafzeit und Tiefschlaf festgestellt werden. Analog zeigt sich jedoch die Abnahme der Anzahl von Bewegungen im Schlaf. In beiden Studien zeigt sich kein signifikanter Effekt auf die Latenz zu Stadium 1 und 2 oder auf die REM-Latenz.

Die Wirkung einer Einzeldosis von $5 \mathrm{mg}$ bzw. $10 \mathrm{mg}$ Olanzapin spiegelt sich in einer Verbesserung der Schlafkontinuität mit verlängerter Gesamtschlafzeit und höherer Schlafeffizienz sowie in einer Zunahme des Tiefschlafs wider (Sharpley et al., 2000). Stadium 1 ist im Vergleich zu Plazebo verkürzt. Unter $10 \mathrm{mg}$ ist ferner die Abnahme des REM-Schlafs bei gleichzeitiger Zunahme der REM-Latenz signifikant.

In einer späteren Studie können Sharpley et al. (2001) auch unter 5 mg Olanzapin erneut eine Zunahme der REM-Latenz, bei allerdings gleichbleibendem REM-Schlaf dokumentieren. Daneben berichten die Autoren eine Zunahme der Schlafkontinuität und des Tiefschlafs bei gleichzeitiger Abnahme des Stadiums 1.

Die Gabe von $25 \mathrm{mg}$ und $100 \mathrm{mg}$ Quetiapin verbessern im Vergleich zu Plazebo die Schlafkontinuität in Form erhöhter Gesamtschlafzeit sowie Schlafeffizienz und führen zu einem höheren Anteil an Stadium 2 (Cohrs et al., 2004b). 
Die Einzelgabe von $1 \mathrm{mg}$ Risperidon führt zu einer Verringerung des REM-Anteils, andere Schlafparameter verbleiben im Wesentlichen unverändert (Sharpley et al., 2003).

$\mathrm{Zu}$ Amisulprid, Aripiprazol, Sertindol und Ziprasidon liegen keinerlei polysomnographische Daten zu diesem Thema vor.

Die atypischen Antipsychotika Clozapin, Olanzapin und Quetiapin verbessern in den meisten Studien die Schlafkontinuität, Risperidon scheint dies dagegen nicht zu tun. Die Wirkungen auf den Tiefschlaf und den REM-Schlaf divergieren. Tabelle 5.1 in der Diskussion zeigt die Auswirkungen atypischer Antipsychotika auf den Schlaf bei gesunden Probanden (und bei Schizophrenen) in der Übersicht. Im Gegensatz zur Wirkung der klassischen Antipsychotika, die sich bei Gesunden anders als bei schizophrenen Patienten widerspiegelt, liegen hier einheitlichere Ergebnisse vor. Möglicherweise sind demzufolge die Effekte atypischer Antipsychotika auf den Schlaf auch eher als primäre Arzneimitteleffekte anzusehen. Zusammengefasst bedeutet dies, dass die atypischen Antipsychotika bei gesunden wie schizophrenen Studienteilnehmern insbesondere Schlafparameter verändern, die sich positiv auf die Schlafkontinuität auswirken.

\subsection{Hypothalamus-Hypophysen-Nebennieren-Achse}

Die Hypothalamus-Hypophysen-Nebennieren-Achse (Hypothalamic-pituitary-adrenal axis, HPA-Achse) stellt eine Verbindung zwischen dem Zentralnervensystem und der Nebenniere dar, deren Kommunikation über spezifische neuroendokrine Hormone geregelt wird. Es besteht ein komplexer Interaktionsmechanismus zwischen dem Hypothalamus, der Hypophyse und der Nebenniere.

Die HPA-Achse reguliert und moduliert vor allem die menschliche Reaktion auf innere und äußere Stressoren. Daneben kontrolliert sie noch verschiedene andere Körperfunktionen, wie z. B. Nahrungsverwertung und Energiebereitstellung. Ferner wirkt sie auf das Immunsystem und die Stimmungslage.

Die wichtigsten Abläufe bezüglich der HPA-Achse sind folgende: Die neuroendokrinen Neurone im Nucleus paraventricularis des Hypothalamus synthetisieren das Kortikotropin-freisetzende Hormon (Corticotropin-releasing hormone, CRH). CRH stimuliert die Sekretion des Adrenokortikotropen Hormons (Adrenocorticotropic hormone, ACTH), das früher als Kortikotropin bezeichnet wurde, aus dem Hypophysenvorderlappen (Syn. Adenohypophyse). ACTH wirkt auf die Nebennierenrinde und sorgt für eine Freisetzung von Kortisol. Dieses wirkt als permissiver Botenstoff in verschiedenen Endorganen und übermittelt so die gewünschte Wirkung aus dem Gehirn an den restlichen Körper. Die Kortisolsekretion unterliegt einem zirkadianen Rhythmus, 
wobei der Kortisolspiegel in den frühen Morgenstunden am höchsten ist. In einem negativen Rückkopplungsmechanismus hemmt Kortisol im Gegenzug die Sekretion von CRH aus dem Hypothalamus und von ACTH aus der Hypophyse (Wuttke, 1997). Dieses ist besonders wichtig zur Aufrechterhaltung der inneren Homöostase. Chronische Belastungen können zu einer Störung in diesem System führen, was sich in einer dauerhaft verminderten oder vermehrten Kortisolsekretion äußern kann (Chrousos und Gold, 1992; Huether, 1996; Mc Ewen, 2003; Walker EF und Diforio, 1997).

Eine Störung in der Regulation der HPA-Achse als wesentlicher pathophysiologischer Bestandteil kann für eine Reihe psychiatrischer Erkrankungen nachgewiesen werden (Rothschild, 2003). Vor allem trifft dies bei einer schweren Depression zu (Holsboer, 2000), aber auch bei der Schizophrenie mehren sich die Hinweise auf das Vorliegen einer solchen Regulationsstörung (Walker EF und Diforio, 1997).

Rückschlüsse auf den Aktivierungsgrad der HPA-Achse können zum einen durch basale Messung der Kortisolkonzentration in Blutplasma, Speichel oder Urin erfolgen. Wie Weinstein et al. (1999) hervorgehoben haben, besteht eine hohe Korrelation zwischen Kortisolmessungen im Blut, Speichel und Urin. Auch die basale ACTH-Konzentration im Blutplasma oder die basale CRH-Konzentration im Liquor cerebrospinalis ${ }^{7} \mathrm{kann}$ gemessen werden.

Um über die Reaktionsfähigkeit Rückschlüsse auf den Aktivierungsgrad der HPAAchse zu ziehen, eignen sich der Dexamethason-Hemmtest ${ }^{8}$ (Mossman und Somoza, 1989; Rothschild, 2003) und der kombinierte Dexamethason-Hemmtest/CRH-Test ${ }^{9}$ (Heuser et al., 1994).

\subsubsection{HPA-Achse bei Depression und bei Schizophrenie}

Viele Studien belegen sowohl erhöhte, basale Kortisolspiegel als auch eine Dexamethason-Nonsuppression im Dexamethason-Hemmtest bei Patienten mit affektiven Störungen (Carroll, 1982; Sachar et al., 1970; Varghese und Brown, 2001).

Eine Dexamethason-Nonsuppression liegt bei etwa $44 \%$ der Patienten mit einer schweren Depression vor (Arana et al., 1985). Mit $67 \%$ ist die Nonsuppressions-Rate bei Patienten mit einer schweren Depression und gleichzeitig bestehenden psychotischen Symptomen deutlich höher (Arana et al., 1985).

\footnotetext{
${ }^{7}$ Gehirn-Rückenmark-Flüssigkeit

${ }^{8}$ Im Dexamethason-Hemmtest werden dem Patienten am Abend 1-2 mg Dexamethason, ein Kortisolderivat, verabreicht. Sind am darauf folgenden Tag die Kortisolwerte im Blut im Gegensatz zum Durchschnittswert bei Gesunden erhöht, spricht man von einer unzureichenden Kortisolsuppression bzw. einer Dexamethason-Nonsuppression.

${ }^{9}$ Dieser Test entspricht weitgehend dem Dexamethason-Hemmtest, zusätzlich werden jedoch am zweiten Tag $100 \mu \mathrm{g}$ CRH intravenös gegeben und neben Kortisol auch ACTH-Werte bestimmt.
} 
In vielen Fällen finden sich erhöhte Kortisolspiegel und/oder eine Nonsuppression auch beim Vorliegen einer Schizophrenie (Walker EF und Diforio, 1997): Zahlreiche Studien können bei Schizophrenen erhöhte Kortisolspiegel im Blutplasma nachweisen (Altamura et al., 1989; Monteleone et al., 1992, 1999; Mück-Seler et al., 1999; Ryan et al., 2003). Erhöhte Kortisolwerte können darüber hinaus bei Jugendlichen mit schizotyper Persönlichkeitsstörung ${ }^{10}$ dokumentiert werden (Weinstein et al., 1999). In einigen Studien hat man allerdings keine erhöhten Kortisolspiegel bei Schizophrenen festgestellt (Kathol et al., 1992; Rao ML et al., 1995).

Pathologische Dexamethason-Hemmtest-Ergebnisse finden sich bei 0-70\% der Schizophrenen, was auf die große Variabilität zwischen den Studien hinweist (Altamura et al., 1989; Asnis et al., 1986; Coppen et al., 1983; Herz et al., 1985; Jakovljević et al., 1998; Lammers et al., 1995; Möller et al., 1986; Mück-Seler et al., 1999; Munro et al., 1984; Sawyer und Jeffries, 1984; Sora et al., 1986; Wik et al., 1986; Yeragani, 1990). Im kombinierten Dexamethason-Hemmtest/CRH-Test zeigen sich erhöhte Kortisolspiegel sowohl bei depressiven als auch schizophrenen Patienten (Heuser et al., 1994; Lammers et al., 1995).

Zusammengefasst liegt höchstwahrscheinlich sowohl bei der Depression als auch bei der Schizophrenie eine erhöhte Aktivität der HPA-Achse vor.

\subsubsection{Zusammenhang zwischen HPA-Achse und Symptomatik bei Schizophrenie}

Zahlreiche Studien weisen auf einen Zusammenhang zwischen erhöhter Aktivität der HPA-Achse und der klinischen Symptomatik schizophrener Patienten hin.

Dabei scheint insbesondere die Ausprägung von Negativsymptomen, depressiven Symptomen, kognitiven Defiziten, internistischen Begleiterkrankungen und in geringerem Umfang auch der Krankheitszustand durch die Aktivität der HPA-Achse beeinflusst zu werden. Auf diese Zusammenhänge soll im Folgenden näher eingegangen werden:

Shirayama et al. (2002) und Zhang et al. (2005) weisen bei schizophrenen Patienten eine Korrelation der Negativsymptomatik mit basal erhöhten Kortisol- und ACTH-Werten im Plasma nach. Die Schwere der Negativsymptomatik ist darüber hinaus auch mit erhöhten Kortisolspiegeln nach Dexamethason-Hemmtest assoziiert

\footnotetext{
${ }^{10}$ Dies ist eine psychiatrische Erkrankung mit exzentrischem Verhalten, Anomalien des Denkens und der Stimmung, die schizophren wirken, obwohl nie eindeutige und charakteristische schizophrene Symptome aufgetreten sind. In einigen Fällen entwickelt sich hieraus im Verlauf eine Schizophrenie.
} 
(Newcomer et al., 1991; Tandon et al., 1989, 1991). Einige Studien können diese Assoziation allerdings nicht feststellen (Addington und Addington, 1990; Ismail et al., 1998; Minas et al., 1990).

Einige Autoren berichten eine Assoziation pathologischer Dexamethason-HemmtestErgebnisse mit dem Ausprägungsgrad depressiver Symptomatik (Ismail et al., 1998; Jones et al., 1994; Munro et al., 1984; Sawyer und Jeffries, 1984) oder mit dem gleichzeitigen Vorliegen einer schweren Depression (Addington und Addington, 1990). Von besonderer klinischer Relevanz ist der Zusammenhang pathologischer Dexamethason-Hemmtest-Ergebnisse bzw. erhöhter basaler Kortisolspiegel bei Schizophrenen mit Suizidversuchen in der Vorgeschichte (Jones et al., 1994; Plocka-Lewandowska et al., 2001). Einige Studien belegen ferner eine Verbesserung depressiver Symptome nach Veränderung der Kortisolspiegel im Blut durch Gabe der Glukokortikoidsynthese-Inhibitoren wie beispielsweise Ketoconazol und Metyrapon (Anand et al., 1995; O'Dwyer et al., 1995; Wolkowitz und Reus, 1999), allerdings sind diese Studien aufgrund geringer Fallzahlen und großer Heterogenität mit Vorsicht zu interpretieren (Wolkowitz und Reus, 1999). Eine Pilotstudie zeigt eine antidepressive Wirkung von Glukokortikoidsynthese-Inhibitoren auch bei Patienten mit Schizophrenie und schizoaffektiver Erkrankung (Marco et al., 2002).

Walder et al. (2000) stellen eine inverse Korrelation von Kortisolspiegeln und kognitiver Leistungsfähigkeit sowohl bei psychiatrischen Patienten, mit und ohne psychotischen Symptomen, als auch bei Gesunden fest. Auch Newcomer et al. (1998) dokumentieren eine Korrelation zwischen erhöhten basalen Kortisolspiegeln mit reduzierter verbaler Gedächtnisleistung bei Schizophrenen. Sauro et al. (2003) können zeigen, dass Stress mit einer glukokortikoiden Aktivierung und Verminderung der deklarativen Gedächtnisfunktion beim Menschen einhergeht. Aber auch die Applikation von Kortisol bei gesunden Probanden, um äußeren Stress zu simulieren, führt zu einer Verminderung der verbalen deklarativen Gedächtnisfunktion (Newcomer et al., 1999). Für Patienten mit einer schweren Depression kann man zeigen, dass eine erfolgreiche Therapie mit einem SSRI zu Verminderung des Kortisolspiegels und zu einer Verbesserung der Gedächtnisfunktion führt (Vythilingam et al., 2004). Auch bei älteren Frauen stellt man in einer longitudinalen Studie eine Assoziation zwischen abnehmenden Kortisolspiegeln und verbesserter Gedächtnisfunktion fest (Seeman TE et al., 1997). Bei Patienten mit einer bipolar affektiven Erkrankung kann neben einem antidepressiven Effekt auch eine Verbesserung der kognitiven Leistungsfähigkeit durch Mifepriston ${ }^{11}$ gezeigt werden (Young et al., 2004). Im Gegensatz hierzu zeigt sich bei Schizophrenen keine Verbesserung der kognitiven Fähigkeit durch Mifepriston (Gallagher et al., 2005).

\footnotetext{
${ }^{11}$ Mifepriston (RU-486) ist ein Progesteron-Rezeptor-Antagonist, der in hohen Dosen ein Antagonist des Glukokortikoid-Rezeptors ist (Young et al., 2004). Klinisch wird dieser Wirkstoff im Präparat Mifegyne ${ }^{\circledR}$ vor allem zum medikamentös induzierten Schwangerschaftsabbruch eingesetzt.
} 
Zahlreiche Studien dokumentieren eine Volumenminderung der Hippocampi bei Schizophrenie (Bogerts et al., 1985; Falkai und Bogerts, 1986; Waldo et al., 1994). Diese Hirnstruktur spielt unter anderem eine große Rolle innerhalb des deklarativen Gedächtnisses (Eichenbaum et al., 1996). In Tierversuchen kann man zeigen, dass länger andauernder Stress und Erhöhungen des Kortisolspiegels zu einem Schaden an Strukturen der Hippocampi führen (Sapolsky et al., 1990). Auch beim Menschen findet man eine Assoziation zwischen erhöhten Kortisolspiegeln bei Patienten mit Cushing Syndrom ${ }^{12}$ und reduziertem Hippocampusvolumen sowie darüber hinaus auch mit einer verminderten Gedächtnisfunktion (Starkman et al., 1992). In einem neueren Übersichtsartikel heben Warrington und Bostwick (2006) die unter Kortisontherapie auftretenden kognitiven Defizite hervor.

Die Über-Aktivierung der HPA-Achse bei schizophrenen Patienten hat auch internistische Auswirkungen. So hebt Dinan (2004) in einem Übersichtsartikel hervor, dass die Über-Aktivierung der HPA-Achse nicht nur bei der Entstehung einer Schizophrenie, sondern möglicherweise auch bei der Entwicklung eines Diabetes Mellitus bei dieser Patientengruppe beteiligt ist. Ryan et al. (2003) zeigen, dass bei bisher unbehandelten Schizophrenen im Vergleich zu Gesunden erhöhte Nüchtern-Glukosewerte und eine vermehrte Insulinresistenz vorkommen. Störungen der Glukoseregulation sind aber auch mit der Einnahme von Antipsychotika an sich in Verbindung gebracht worden (Holt und Peveler, 2006; Newcomer et al., 2002). Im Vergleich zur Normalbevölkerung sind Hyperglykämie und Diabetes Mellitus Typ II häufiger bei Schizophrenen unter antipsychotischer Therapie anzutreffen (Newcomer et al., 2002). Thakore et al. (2002) und Ryan et al. (2004) dokumentieren neben erhöhten Kortisolspiegeln einen erhöhten abdominellen Fettgewebsanteil bei Schizophrenen im Vergleich zu gesunden Probanden. Ferner besteht auch bei depressiven Patienten eine Assoziation zwischen erhöhtem Kortisolspiegel und internistisch relevanten Erkrankungen, wie beispielsweise Bluthochdruck, erhöhtem abdominellen Fettgewebsanteil, Magengeschwüren, Diabetes und verminderter Knochendichte (Brown et al., 2004).

Darüber hinaus korreliert ein unter Therapie mit typischen Antipsychotika persistierender pathologischer Dexamethason-Hemmtest als Zeichen erhöhter Aktivität der HPA-Achse mit einem schlechteren Krankheitszustand nach einem Jahr bei Schizophrenen (Tandon et al., 1991).

\subsection{Wirkung der Antipsychotika auf die HPA-Achse}

Ähnlich wie bei den Ausführungen zur Wirkung der Antipsychotika auf den Schlaf (vgl. Abschnitt 2.3) muss kritisch angemerkt werden, dass auch die Aussagekraft der

\footnotetext{
${ }^{12}$ Dieses Syndrom bezeichnet einen Symptomenkomplex, der durch das Überangebot von Glukokortikoiden im Organismus gekennzeichnet ist.
} 
Studien, die sich mit der Wirkung von Antipsychotika auf die HPA-Achse auseinandergesetzt haben, aus methodischen Gründen (sehr kleine Fallzahlen, keine PlazeboVergleiche) in vielen Fällen eingeschränkt ist. Darüber hinaus gibt es insgesamt nur sehr wenige Studien, die die Auswirkungen der atypischer Antipsychotika auf die HPA-Achse untersucht haben.

\subsubsection{Wirkung klassischer Antipsychotika bei schizophrenen Patienten}

Es finden sich unter der Therapie schizophrener Patienten mit klassischen Antipsychotika wie beispielsweise Chlorpromazin, Haloperidol, Sulpirid und Perphenazin erniedrigte (Wik, 1995), gleich bleibende (Saldanha et al., 1972; Wik et al., 1986), aber auch erhöhte basale Kortisolspiegel (Cesková et al., 2001). Zudem kann man zeigen, dass eine länger dauernde Haloperidol-Therapie zu einer Verminderung der ACTH-Sekretion führt (Brambilla et al., 1975).

Ein plötzliches Absetzten von klassischen Antipsychotika führt dagegen zur Erhöhung der Kortisolspiegel (Naber et al., 1985).

Unter klassischer antipsychotischer Therapie (beispielsweise mit Haloperidol, Thiothixen, Chlorpromazin) normalisiert sich in vielen Fällen der Dexamethason-Hemmtest, was meist auch mit einer Besserung der schizophrenen Symptomatik verbunden ist (Möller et al., 1986; Tandon et al., 1991; Wik et al., 1986).

Weitere Studien prüften die Auswirkungen von Antipsychotika auf die Kortisolspiegel bzw. ACTH-Spiegel nach vorheriger Stimulation der HPA-Achse durch verschiedene Substanzen oder Stressoren. Fluphenazin und Haloperidol können eine durch M-Chlorphenylpiperazin (M-CPP) ${ }^{13}$ hervorgerufene vermehrte Sekretion von ACTH (Kahn et al., 1993) oder Kortisol (Owen et al., 1993) nicht abschwächen.

In der Mehrzahl der Studien weisen die klassischen Antipsychotika eine kortisolsenkende Wirkung auf. Allerdings zeigt sich dieser Effekt nicht in jedem Fall und häufig ist dieser auch nicht besonders ausgeprägt.

\subsubsection{Wirkung atypischer Antipsychotika bei schizophrenen Patienten}

In Studien zur Wirkung atypischer Antipsychotika bei schizophrenen Patienten zeigt sich, dass sowohl Clozapin (Meltzer, 1989), Olanzapin (Mann et al., 2006; Ryan et al., 2004) als auch Risperidon (Ryan et al., 2004; Zhang et al., 2005) zur Senkung des

\footnotetext{
${ }^{13} \mathrm{M}-\mathrm{CPP}$ ist ein nicht-selektiver, direkter $5-\mathrm{HT}_{2}$-Agonist.
} 
Kortisolspiegels führen. In den meisten Fällen ist dies zudem mit einer Besserung krankheitsspezifischer Symptome verbunden.

Untersuchungen zu den Auswirkungen atypischer Antipsychotika auf den Dexamethason-Hemmtest sind bisher nicht vorhanden.

Der Wechsel von einem klassischen Antipsychotikum zu Clozapin führt in mehreren Studien zur Senkung der Kortisolspiegel (Hatzimanolis et al., 1998; Markianos et al., 1999b), allerdings nicht in allen (Breier et al., 1994). In einer Studie von Markianos et al. (1999a) hat die Umstellung von Haloperidol auf Risperidon keine Veränderung der Kortisolspiegel zufolge.

Clozapin schwächt die Stimulation der Kortisolsekretion durch Gabe von MK-212 ${ }^{14}$ (Meltzer, 1989), Fenfluramin ${ }^{15}$ (Curtis et al., 1995) oder M-CPP (Kahn et al., 1993, 1994; Owen et al., 1993) ab, was sich in einer Senkung der Kortisolspiegel bzw. der ACTH-Spiegel ausdrückt. Auch Olanzapin blockiert die durch M-CPP induzierte Freisetzung von Kortisol und ACTH (Scheepers et al., 2001).

Die Ergebnisse der Studien, die sich mit dem Einfluss atypischer Antipsychotika beschäftigt haben, sind einheitlicher als diejenigen, die den Einfluss klassischer Antipsychotika bei schizophrenen Patienten untersucht haben. Zusammengefasst scheinen die atypischen Antipsychotika Clozapin, Olanzapin und Risperidon die Aktivität der HPA-Achse abzuschwächen. Tabelle 5.3 in der Diskussion stellt die Auswirkungen atypischer Antipsychotika bei schizophrenen Patienten (und bei Gesunden) in der Übersicht dar. Studien zu Amisulprid, Aripiprazol, Sertindol, Quetiapin und Ziprasidon liegen bisher nicht vor.

\subsubsection{Wirkung klassischer Antipsychotika bei gesunden Probanden}

Die klassischen Antipsychotika wie beispielsweise Haloperidol und Sulpirid haben vorwiegend keinen Effekt auf die basalen Kortisolspiegel gesunder Probanden (Baptista et al., 1997a,b; Cohrs et al., 2006; De Koning und De Vries, 1995; Laakmann et al., 1984; Von Bahr et al., 1991). Im Gegensatz hierzu zeigt sich unter Haloperidol auch ein Anstieg des Kortisolspiegels, allerdings nur in zwei Studien derselben Arbeitsgruppe (Murburg et al., 1986, 1993).

In einer weiteren Studie blockiert Haloperidol einen durch Hitzestress induzierten Kortisolanstieg (Hennig et al., 1995).

\footnotetext{
${ }^{14} \mathrm{MK}-212$ ist ein nicht-selektiver, direkter 5 -HT ${ }_{2}$-Agonist.

${ }^{15}$ Fenfluramin ist ein indirekter 5 -HT-Agonist.
} 
Die klassischen Antipsychotika scheinen bei Gesunden zumeist keinen Effekt auf die Kortisolsekretion zu haben. Werden gesunde Probanden durch einen äußeren Stressor gereizt, weisen sie allerdings auch eine kortisolsenkende Wirkung auf.

\subsubsection{Wirkung atypischer Antipsychotika bei gesunden Probanden}

Die atypischen Antipsychotika Olanzapin und Quetiapin bewirken eine Senkung des basalen Kortisolspiegels und des ACTH-Spiegels bei gesunden Probanden (Cohrs et al., 2004a, 2006). Eine andere Arbeitsgruppe dokumentiert unter Quetiapin nur gesenkte Kortisolspiegel, nicht jedoch erniedrigte ACTH-Spiegel (De Borja Gonçalves Guerra et al., 2005). Dies führen De Borja Gonçalves Guerra et al. (2005) allerdings zum Teil auf eine mangelnde Sensitivität des verwendeten Hormon-Assays zurück. Eine Studie an acht männlichen Probanden beschreibt unveränderte Kortisolspiegel nach intravenöser Gabe von 20 bzw. $100 \mathrm{mg}$ Amisulprid (Wetzel et al., 1994).

Sowohl über unveränderte als auch über erniedrigte Kortisolspiegel wird nach Gabe von atypischen Antipsychotika berichtet. Für Aripiprazol, Clozapin, Risperidon, Sertindol und Ziprasidon liegen bisher keinerlei Daten vor. Die Mehrzahl der Studien weist auf eine überwiegende Senkung der Kortisolsekretion hin. Tabelle 5.3 in der Diskussion gibt einen Überblick über die Auswirkungen atypischer Antipsychotika auf den Kortisolspiegel gesunder Personen und schizophrener Patienten.

\subsection{Fragestellung}

Schizophrenie und Depression gehen in den meisten Fällen mit Schlafstörungen einher, teilweise gehen diese den Erkrankungen sogar voraus oder sind Anzeichen eines Rückfalls (vgl. Abschnitt 2.2.3 und 2.2.4). Anhand polysomnographischer Untersuchungen kann gezeigt werden, dass der Schweregrad der Schizophrenie mit einer erhöhten Wachzeit, mit reduziertem Tiefschlaf- und REM-Anteil sowie mit einer verkürzten REM-Latenz korreliert (Benson und Zarcone, 2000; Kempenaers et al., 1988; Tandon et al., 1992). Zudem besteht ein Zusammenhang zwischen vermindertem Tiefschlaf bzw. verkürzter REM-Latenz und dem Ausmaß der Negativsymptomatik (Ganguli et al., 1987; Keshavan et al., 1995a; Tandon et al., 1992; Taylor et al., 1991) sowie einem schlechteren Krankheitsverlauf (Goldman et al., 1996; Keshavan et al., 1995b; Tandon et al., 2000) (vgl. Abschnitt 2.2.6). 
Zahlreiche Studien belegen eine erhöhte Aktivität der HPA-Achse bei der Depression, aber auch bei der Schizophrenie (vgl. Abschnitt 2.4.1). Dabei besteht eine Assoziation zwischen der erhöhten Aktivität der HPA-Achse und der Ausprägung von Negativsymptomen, depressiven Symptomen, kognitiven Defiziten und internistischen Begleiterkrankungen (Jones et al., 1994; Ryan et al., 2004; Thakore et al., 2002; Walder et al., 2000; Zhang et al., 2005) (vgl. Abschnitt 2.4.2).

Im Gegensatz zu klassischen Antipsychotika zeichnen sich die atypischen Antipsychotika insbesondere durch ihre bessere Wirkung auf Negativsymptome, depressive Symptome und kognitive Defizite aus. Damit wirken sie auf einige Symptome, die mit einer Überaktivierung der HPA-Achse und mit einem gestörten Schlaf in Zusammenhang stehen.

Möglicherweise ist der Effekt dieser Medikamente auf die klinische Symptomatik unter anderem durch ihre Wirkung auf den Schlaf und die HPA-Achse zu erklären. Derzeit gibt es allerdings nur wenige Studien, die den Einfluss atypischer Antipsychotika auf den polysomnographisch erfassten Schlaf oder auf die Hormone der HPA-Achse untersucht haben. Um zunächst die primären Auswirkungen der atypischen Antipsychotika zu untersuchen, sind Studien an gesunden Probanden, ähnlich wie in der vorliegenden Studie zu den Effekten Ziprasidons, nötig.

Weitere Forschung ist zudem erforderlich, da die Antipsychotika in wachsendem Ausmaß auch als Schlafmittel eingesetzt werden. Die Anzahl der verschriebenen Antipsychotika nahm so in den Jahren 1986-1995 stetig zu, während die potentiell abhängig machenden Benzodiazepine zunehmend weniger verschrieben wurden (Linden und Thiels, 2001).

Für die klassischen Antipsychotika gilt im Allgemeinen, dass schwachpotente Antipsychotika, die eine hohe Dosierung in mg erfordern, auch stärker sedierend wirken als hochpotente Antipsychotika, die nur eine niedrige Dosierung erfordern. Dieses Prinzip gilt in gewissem Umfang auch für die atypischen Antipsychotika. So ist das hochpotente, niedriger zu dosierende Risperidon weniger sedierend als die niedrigerpotenten, höher zu dosierenden Antipsychotika Quetiapin und Clozapin (Miller, 2004). Für Olanzapin gilt diese Regel allerdings nicht, denn obwohl es hochpotent und niedrig zu dosieren ist, wirkt es sedierender als Ziprasidon. Dieser relativ ausgeprägte, sedierende Effekt von Olanzapin lässt sich durch sein Rezeptorprofil erklären. Olanzapin besitzt im Vergleich zu Clozapin, Ziprasidon, Risperidon und Quetiapin die höchste Affinität zum menschlichen $\mathrm{H}_{1}$-Histamin-Rezeptor (Miller, 2004; Richelson und Souder, 2000). Somit ist einerseits die Dosierung bzw. der zentral wirksame Anteil der Medikation und anderseits die Affinität zum $\mathrm{H}_{1}$-Histamin-Rezeptor für die sedierende Wirkung verantwortlich (Miller, 2004).

In üblicher Dosierung zeigt Clozapin eine deutliche Sedierung. Quetiapin und Olanzapin weisen einen moderaten, Risperidon und Ziprasidon einen milden sedierenden 
Effekt auf (Miller, 2004). Bei Gesunden zeigt sich ein solcher milder sedierender Effekt bereits nach oraler Gabe von 5 bis $20 \mathrm{mg}$ Ziprasidon (Miceli et al., 2000). Ferner besitzen die atypischen Antipsychotika beruhigende Effekte bei agitierten und schlafgestörten Patienten mit psychischen Erkrankungen (Adityanjee und Schulz, 2002; Daniel et al., 2001; Marder, 2006). Bei Ziprasidon tritt laut einer Studie von Daniel et al. (2001) die beruhigende Wirkung bei agitierten Patienten nach intramuskulärer Injektion von $20 \mathrm{mg}$ ein. In der Fachinformation zu Ziprasidon sind Sedierung und Somnolenz zudem als häufige ${ }^{16}$ Nebenwirkungen angegeben (Pfizer, 2006). Im Gegensatz hierzu werden allerdings auch das Auftreten von Unruhe als häufig und das Vorkommen von Agitiertheit, Angst und Alpträumen als gelegentlich beschrieben.

Ursächlich mag dafür das besondere Rezeptorprofil Ziprasidons sein. Wie andere atypische Antipsychotika ist Ziprasidon ein 5- $\mathrm{HT}_{2} / \mathrm{D}_{2}$-Rezeptor-Antagonist (Stahl und Shayegan, 2003). Neben der Wirkung auf weitere 5-HT-Rezeptoren hemmt es allerdings noch die Wiederaufnahme von Serotonin und Noradrenalin und wirkt auf den $\alpha_{1}$-Adrenozeptor, den $\mathrm{H}_{1}$-Histamin-Rezeptor und den muskarinergen AcetylcholinRezeptor vom Typ $\mathrm{M}_{1}$ (vgl. Abschnitt 2.1.2).

Für die sedierenden Effekte Ziprasidons mag der $\mathrm{H}_{1}$-Histamin-Rezeptor-Antagonismus verantwortlich sein. Daneben gibt es Hinweise, dass 5- $\mathrm{HT}_{2}$-Antagonisten zu einer Erhöhung des Tiefschlafs führen (Idzikowski et al., 1986; Paiva et al., 1988; Sharpley et al., 1994, 2000). Auch Ziprasidon könnte somit über seinen 5-HT ${ }_{2}$-Antagonismus zu einer Erhöhung des Tiefschlafs führen. Nicht alle atypischen Antipsychotika erhöhen jedoch den Tiefschlaf (vgl. Abschnitt 2.3), obwohl sie 5-HT - -Antagonisten sind. Das Auftreten von Unruhe und Agitiertheit könnte durch die Hemmung der Wiederaufnahme von Serotonin und Noradrenalin verursacht werden, was als häufige Nebenwirkung unter der Therapie mit SSRIs oder SNRIs vorkommt (Möller et al., 2001). Zudem erniedrigen SSRIs die Gesamtschlafzeit und Schlafeffizienz (Sharpley und Cowen, 1995). Da keine polysomnographischen Studien zu Ziprasidon vorliegen, kann nicht abschließend geklärt werden, wie sich Ziprasidon auf die verschiedenen Schlafparameter auswirkt.

Viele atypische Antipsychotika üben einen abschwächenden Einfluss auf die Aktivität der HPA-Achse aus (vgl. Abschnitt 2.5). Die Auswirkungen Ziprasidons auf die HPA-Achse sind bisher in keiner Studie untersucht worden. In der Literatur gibt es Hinweise darauf, dass die Senkung des Kortisolspiegels vor allem auf einem $5-\mathrm{HT}_{2}$ Antagonismus beruht (Meltzer, 1989; Scheepers et al., 2001). Im Gegensatz hierzu bewirken 5- $\mathrm{HT}_{1 \mathrm{~A}}$-Agonisten und Medikamente, welche die Wiederaufnahme von Serotonin und Noradrenalin inhibieren, eher eine gesteigerte Aktivität der HPA-Achse (Contesse et al., 2000; Laakmann et al., 1990). Anhand der begrenzten Studienlage kann vor Beginn der experimentellen Urinuntersuchung nicht abgeschätzt werden, wie

\footnotetext{
${ }^{16}$ Nebenwirkungen werden nach ihrer Häufigkeit in sehr häufig $(>1 / 10)$, häufig $(>1 / 100,<1 / 10)$, gelegentlich $(>1 / 1000,<1 / 100)$ und selten $(<1 / 1000)$ eingeteilt.
} 
sich Ziprasidon auf die Kortisolexkretion auswirkt. Sowohl eine Reduktion als auch eine Erhöhung der Kortisolexkretion ist aufgrund des beschriebenen Rezeptorprofils vorstellbar.

Im Rahmen der vorliegenden Studie sollten daher an gesunden Probanden folgende Fragen geklärt werden:

1. Wie wirkt sich Ziprasidon auf den polysomnographisch erfassten Schlaf aus?

2. Wie wirkt sich Ziprasidon auf die Kortisolexkretion aus? 


\section{Kapitel 3}

\section{Methodik}

\subsection{Art und Ort der Studie}

Die vorliegende Studie wurde in einem doppel-blinden, randomisierten, plazebo-kontrollierten, cross-over und single-center Design durchgeführt. Die Wirkung von $40 \mathrm{mg}$ Ziprasidon auf den ungestörten und den akustisch gestörten Schlaf sowie auf die nächtliche Kortisolexkretion wurde bei gesunden Probanden erfasst.

Die Studie beinhaltete polysomnographische, testpsychologische und laborchemische Untersuchungen und diente der Grundlagenforschung. Sie wurde im Labor für Schlafmedizin der Abteilung für Psychiatrie und Psychotherapie des Bereichs Humanmedizin der Georg-August-Universität Göttingen von April bis Juli 2004 durchgeführt. Die Kortisolkonzentration der Urinproben wurde im Neurobiologischen Labor der Abteilung für Psychiatrie und Psychotherapie der Universität Göttingen gemessen.

\subsection{Untersuchungspopulation}

Die männlichen Probanden meldeten sich auf entsprechende Aushänge freiwillig zur Studie. Eine Gesamtpopulation von $N=16$ wurde angestrebt. Nach Abschluss der gesamten Studie erhielten die Probanden eine Aufwandsentschädigung in Höhe von 300 Euro oder einen anteiligen Betrag bei vorzeitigem Ausscheiden aus der Studie.

\subsection{Einschluss-, Ausschluss- und Abbruchkriterien}

Die Teilnahme an der Studie war auf gesunde Freiwillige im Alter von 18 bis 40 Jahren beschränkt. Bevor die Probanden an der Studie teilnehmen konnten, wurden sie maximal zwei Wochen vor Studienbeginn eingehend untersucht. 
Die Untersuchung beinhaltete eine Anamnese und körperliche Untersuchung einschließlich venöser Blutentnahme unter Nüchternbedingungen mit Bestimmung von kleinem Blutbild ${ }^{1}$, den Elektrolyten Natrium und Kalium, Glukose, den Nierenfunktionsparametern Harnstoff und Kreatinin sowie den Leberfunktionsparametern Aspartataminotransferase (AST), Alaninaminotransferase (ALT) und $\gamma$-Glutamyltranspeptidase $(\gamma-\mathrm{GT})$. Die Werte wurden im Zentrallabor des Universitätsklinikums Göttingen bestimmt.

Ferner wurde der Mittelstrahlurin der Probanden im Labor der Abteilung für Psychiatrie und Psychotherapie der Universität Göttingen untersucht. Es wurden der Urinstatus und das Urinsediment bestimmt sowie ein Drogenscreening durchgeführt. Im Drogenscreening wurden folgende Stoffe erfasst: Amphetamine, Barbiturate, Benzodiazepine, Kokain-Metabolite, Opiate, Cannabinoide und Methadon.

Ferner wurden bei jedem Probanden ein Elektrokardiogramm (EKG) und ein Elektroenzephalogramm (EEG) abgeleitet.

Die Probanden wurden mindestens drei Nächte vor Beginn der Studie während einer Screeningnacht polysomnographisch im Schlaflabor auf das Vorliegen einer schlafmedizinisch relevanten Erkrankung untersucht. Ein Schlafapnoesyndrom (Apnoe-Hypopnoe-Index, AHI > 10) oder ein Syndrom periodischer Beinbewegungen im Schlaf (Periodic movements in sleep-Index, PMS-Index > 10) während der Screeningnacht waren Ausschlusskriterien für die Teilnahme an der vorliegenden Studie.

Von der Studienteilnahme ausgeschlossen wurden Probanden mit einer der folgenden psychiatrischen oder neurologischen Erkrankungen: Insomnie, bipolar affektive Störung, Schizophrenie, wahnhafte Störung, Epilepsie, schwere Depression, Zwangserkrankung, Sozialphobie, Alkohol- oder Drogenabhängigkeit. Ein Ausschluss erfolgte ebenfalls bei einer ernsthaften internistischen Erkrankung, bei bekannter Überempfindlichkeit gegen Ziprasidon, bei kardiovaskulärer (Z. n. Myokardinfarkt, Herzinsuffizienz und Reizleitungsstörungen) oder zerebrovaskulärer Erkrankung, bei schwerem Schädelhirntrauma in der Vorgeschichte, bei regelmäßiger Medikamenteneinnahme, Leberinsuffizienz sowie bei Zuständen, die mit Hypotonie einhergehen, wie Dehydrierung, Hypovolämie oder Behandlung mit Antihypertensiva.

Während der letzten vier Wochen vor Studienbeginn durfte keiner der Probanden an einer anderen klinischen Studie teilgenommen oder eine Reise mit Zeitzonenwechsel unternommen haben.

Vor Beginn der Studie wurde jeder Proband über den Ablauf sowie über die möglichen Risiken und Nebenwirkungen der Studie aufgeklärt. Abschließend musste eine Einverständniserklärung unterschrieben werden.

\footnotetext{
${ }^{1}$ Hierzu gehören die Parameter: Hämoglobinkonzentration, Hämatokritwert, Erythrozytenanzahl, MCV, MCH, MCHC, Thrombozyten- und Leukozytenanzahl.
} 
Zum Studienabbruch führte das Auftreten von schwerwiegenden unerwünschten Wirkungen durch das verabreichte Pharmakon. Die Probanden konnten jederzeit die Teilnahme an der Studie beenden.

\subsection{Ethik}

Die Ethikkommission der Georg-August-Universität genehmigte die Studie unter der Antragsnummer 18/2/04 am 30.03.2004.

Eine wesentliche Grundlage bildete dabei die Deklaration von Helsinki des Weltärztebundes (Deklaration von Helsinki, 2004).

\subsection{Studienablauf}

Die Probanden hielten sich an zwei aufeinander folgenden Tagen im Abstand von einer Woche im Schlaflabor auf. Dazwischen lag jeweils eine pharmakologische Auswaschphase von fünf Tagen. Als Ernährung wurde während der Untersuchungstage fleischfreie Krankenhauskost gereicht.

Während der ersten Nacht (Nacht 1) wurden die polysomnographischen Zielparameter unter ungestörten Bedingungen erfasst. In der darauf folgenden Nacht (Nacht 2) wurde der Schlaf durch akustische Reize gestört.

Die Prüfmedikation (40 mg Ziprasidon oder Plazebo) wurde in doppel-blinder Weise verabreicht und sowohl in Nacht 1 als auch Nacht 2 einer jeden Untersuchungseinheit eingenommen. Die Reihenfolge, ob in der ersten bzw. zweiten Untersuchungseinheit Ziprasidon oder Plazebo gegeben wurde, war randomisiert. Die Tabelle 3.1 zeigt den Studienablauf schematisch.

Die Probanden nahmen die Prüfmedikation zwei Stunden vor dem Zubettgehen ein. Vor der Einnahme des Medikaments und nach dem morgendlichen Erwachen wurde eine Puls- und Blutdruckkontrolle vorgenommen.

Jeweils am Morgen nach einer Untersuchungsnacht füllten die Probanden kurz nach dem Erwachen die standardisierten Fragebögen, Schlaffragebogen-A (SF-A) nach Görtelmeyer (1986) und die „Visuelle Analogskala morgens“ (VIS-M) nach Ott et al. (1986), zur Erfassung des subjektiven Schlafbefindens aus.

Die beiden Fragebögen wurden von den Probanden kurz nach dem Erwachen ausgefüllt. 
Tabelle 3.1: Schematische Darstellung des Studienablaufs

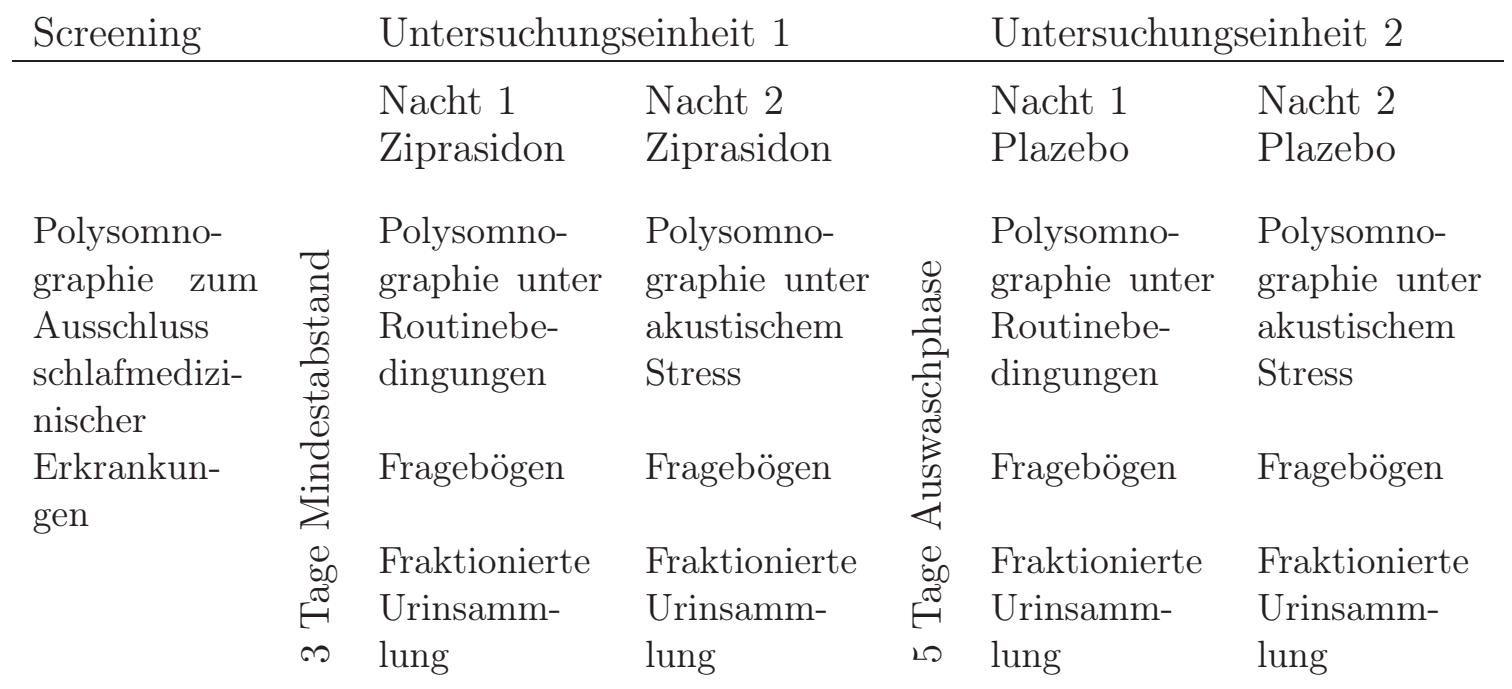

Zur Bestimmung der Kortisolexkretion wurde der Urin fraktioniert in bestimmten Zeiträumen gesammelt (siehe Abschnitt 3.5.3).

Die Bettzeit (Time in bed, TIB) betrug üblicherweise acht Stunden und erstreckte sich von 23-7 Uhr. Die Probanden wurden jeweils vom Untersucher geweckt. Tagsüber hielten sich die Probanden im Schlaflabor auf, wobei es Ihnen untersagt war, sich zum Schlafen niederzulegen. Nach der zweiten Nacht hielten sich die Probanden bis zum Mittag im Schlaflabor auf.

\subsubsection{Prüfmedikation}

Bei gesunden Probenden zeigt sich bereits ein milder sedierender Effekt nach oraler Gabe von 5 bis $20 \mathrm{mg}$ Ziprasidon (Miceli et al., 2000). Zur Behandlung einer Schizophrenie sind höhere Dosen von etwa 80 bis $160 \mathrm{mg}$ erforderlich (Daniel et al., 1999; Goff et al., 1998; Kane, 2003). Aus diesem Grund wurden $40 \mathrm{mg}$ Ziprasidon wurden als intermediäre Testdosis gewählt.

Ziprasidon wird bei Darreichung in Kombination mit einer fetthaltigen Mahlzeit besser aufgenommen (Hamelin et al., 1998). Der maximale Ziprasidonspiegel im Blut wird erst etwa vier bis fünf Stunden nach oraler Einnahme erreicht (Miceli et al., 2000). Um eine ausreichende Ziprasidonkonzentration im Blut zum Einschlafzeitpunkt zu erhalten, wurde Ziprasidon zwei Stunden vor der Bettzeit in Kombination mit einer kleinen Spätmahlzeit verabreicht. 
Die Prüfmedikation stammte aus der Apotheke des Universitätsklinikums Göttingen. Dort war sie zuvor randomisiert und verblindet worden.

\subsubsection{Insomnie-Modell}

Die Applikation von akustischen Reizen während der Schlafphase führt zu Schlafstörungen und kann so als Insomnie-Modell dienen. Die experimentelle Störung des Schlafs erfolgte aber auch, um einen Deckeneffekt zu vermeiden. Bei gesunden, gut schlafenden Probanden sollte ein Effekt hinsichtlich der vermuteten schlafverbessernden Eigenschaften der Medikation gezeigt werden können. Daneben sollte der akustische Stress einen Bodeneffekt bezüglich der Kortisolexkretion abschwächen. Der Bodeneffekt beschreibt dabei eine durch ein Medikament nicht weiter zu senkende, nächtliche Kortisolexkretion.

Jeweils während der zweiten Untersuchungsnacht einer Untersuchungseinheit wurde der Schlaf mittels akustischer Reize gestört, wie bereits in anderen Studien in ähnlicher Weise zuvor (Cluydts et al., 1995; Cohrs et al., 2004b). Zur Vergleichbarkeit der Untersuchungsbedingungen wurden die Töne nach einem festen Schema mittels Lautsprecher in die individuellen Schlafräume geschickt. Zur Tonerzeugung wurde die Kompositionssoftware CUBASIS VST 3.0 (Steinberg, Hamburg, Deutschland) verwendet.

Das Tonprogramm wurde zu Beginn der Bettzeit gestartet und nach dem Erwecken des Probanden beendet. In den Nächten mit Tonexposition stand für jeden Probanden das gleiche Tonprogramm zur Verfügung. Während der achtstündigen Bettzeit erklangen Folgen von gleichartigen Klaviertönen in Stakkato. Die Töne traten in unregelmäßigen Zeitabständen, d. h. etwa alle 30-90 Sekunden auf, dauerten ca. 4-5 Sekunden lang an und variierten jeweils in ihrer Tonhöhe zwischen 880-3520 Hz und in ihrer Lautstärke zwischen $55-85 \mathrm{~dB}(\mathrm{~A})$.

\subsubsection{Fraktionierte Urinsammlung}

Eine venöse Blutentnahme stellt einen Stressfaktor dar, der möglicherweise zu veränderten Kortisolspiegeln im Blut führt. Im Gegensatz dazu ist die Gewinnung von Urin mit weniger psychophysiologischem Stress für die Probanden verbunden. Die Kortisolkonzentration im Urin spiegelt die Kortisolwerte im Blutserum hinreichend wider (Doering et al., 2001; Weinstein et al., 1999). Außerdem bietet sich auf diese Weise die Möglichkeit, einen Durchschnitt der variierenden Kortisolausscheidung in einem Intervall von mehreren Stunden nichtinvasiv zu ermitteln. Äußere Störeffekte auf die Kortisolsekretion lassen sich tagsüber kaum vermeiden, während der nächtlichen Ruhezeit sind sie jedoch minimiert. Aus diesen Gründen erfolgte die Bestimmung der 
Kortisolexkretion in fraktioniertem Sammelurin in definierten Sammelperioden vor und nach der nächtlichen Schlafenszeit. Die erste Urinportion erfasste den Zeitraum von 19-23 Uhr, die zweite den Nachturin von 23-7 Uhr und die dritte den Urin vom Vormittag von $7-11 \mathrm{Uhr}$.

Die Probanden mussten vor Beginn der Sammelperiode die Blase entleeren und den Urin verwerfen, anschließend erfolgte die Urinsammlung. Hierzu erhielten die Probanden jeweils vor Beginn einer Sammelperiode ein persönliches Urinsammelbehältnis. In den Urinsammelbehältnissen befanden sich bereits jeweils vorbereitet $0,5 \mathrm{~g}$ EDTADinatriumsalz und $0,5 \mathrm{~g}$ Natriumdisulfit $\left(\mathrm{Na}_{2} \mathrm{~S}_{2} \mathrm{O}_{5}\right)$ als Urinstabilisatoren. Das Gesamtvolumen des Probandenurins wurde sogleich nach Abschluss einer Sammelperiode bestimmt. Anschließend wurde der Urin in verschiedenen Aliquots bei einer Temperatur von $-35^{\circ} \mathrm{C}$ bis zur laborchemischen Untersuchung eingefroren.

\subsection{Sicherheitsverlaufsparameter}

Für jeden Probanden wurde ein Studienordner mit allen zur Falldokumentation erforderlichen Unterlagen angelegt. Nach Abschluss einer Untersuchungseinheit wurde bei den Probanden eine körperliche Untersuchung durchgeführt. Ferner erfolgte eine Blutentnahme mit Bestimmung von Routinelaborparametern (kleines Blutbild, Elektrolyte, Nieren- und Leberfunktionsparameter) und eine EKG-Ableitung.

Zur Dokumentation möglicher unerwünschter Arzneimittelwirkungen oder sonstiger Auswirkungen der Untersuchungsbedingungen wurde die „Fischer Somatische Symptome oder Unerwünschte Effekte Check List" (FSUCL) (Fischer-Cornelssen, 1986) ausgefüllt.

\subsection{Untersuchungsverfahren}

\subsubsection{Beurteilung der objektiven Schlafqualität (Polysomnographie)}

Die polysomnographische Untersuchung zur Erfassung der Schlafparameter erfolgte nach den international anerkannten Richtlinien von Rechtschaffen und Kales (1968) und den Richtlinien der Deutschen Gesellschaft für Schlafmedizin (Penzel et al., 1993). Die Untersuchung umfasste: zwei EEGs mit Ableitung von C4 gegen A1 und C3 gegen A2 bezogen auf das 10-20-System, zwei EOGs mit Ableitung vom linken und rechten Epikanthus, ein submentales EMG und jeweils ein EMG des linken bzw. rechten M. tibialis anterior sowie ein einkanaliges EKG. 
Um Probanden mit einer schlafbezogenen Atmungsstörung zu entdecken, wurde während der Sreeningnacht zusätzlich der Atemfluss mittels Thermoelementen an Mund und Nase gemessen sowie die thorakalen- und abdominellen Atembewegungen anhand von Induktionsplethysmographie registriert. Zudem wurde die periphere arterielle Sauerstoffsättigung durch Pulsoxymetrie erfasst.

Die polysomnographische Aufzeichnung fand unter Verwendung der Software Leonardo (MKE Medizintechnik für Kinder und Erwachsene GmbH, Willroth, Deutschland) statt. Die Nächte wurden visuell nach den standardisierten Regeln von Rechtschaffen und Kales (1968) ausgewertet.

Die Bestimmung der REM-Dichte erfolgte visuell in semiquantitativer Weise (Penzel et al., 1993). Hierzu wurde eine übliche REM-Schlafepoche mit einer Dauer von 30 Sekunden in zehn Mini-Epochen unterteilt. Anschließend wurde für jede einzelne Mini-Epoche beurteilt, ob rasche Augenbewegungen vorkamen. Hieraus resultierte ein Wert zwischen null und zehn. Es wurde die mittlere REM-Dichte aller REM-Perioden (REM-Dichte) und die der ersten REM-Periode (REM-Dichte-1) bestimmt.

Das EMG der Mm. tibiales anteriores diente der Erkennung und Quantifizierung periodischer Beinbewegungen im Schlaf (Periodic leg movements in sleep, PLMS).

Anhand der den einzelnen Schlafepochen zugeordneten Stadien wurden die in Tabelle 3.2 aufgeführten Schlafparameter berechnet. Die Schlafparameter wurden aufgrund der Internationalität des Fachgebiets teilweise mit englischsprachigen Begriffen bezeichnet.

\subsubsection{Beurteilung der subjektiven Schlafqualität (Fragebögen)}

Neben der objektiven Schlafqualität sollte im Rahmen dieser Studie auch die subjektive Schlafqualität erfasst werden.

Die Beurteilung der subjektiven Schlafqualität kann vornehmlich durch standardisierte Fragebögen (Nowell et al., 2000) geschehen. In der Leitlinie S2 „Nicht erholsamer Schlaf" der Deutschen Gesellschaft für Schlafforschung und Schlafmedizin (DGSM) sind Fragebogen- und Interviewinstrumente genannt, die in deutschen Versionen zur Verfügung stehen und die den testtheoretischen Kriterien der Reliabilität, Validität und Objektivität genügen (Fischer et al., 2001). Dazu zählen auch die in der vorliegenden Studie verwendeten Fragebögen, nämlich der Schlaffragebogen-A (SF-A) nach Görtelmeyer (1986) und die „Visuelle Analogskala morgens“ (VIS-M) nach Ott et al. (1986).

Der SF-A setzt sich aus 22 Fragen zusammen und bewertet den Schlaf der vorausgegangenen Nacht. Die Auswertung des Fragebogens folgt einem festen Schema. Dabei werden die Parameter Schlafqualität (SQ), das Gefühl des Erholtseins nach 
Tabelle 3.2: Die Standardschlafparameter und ihre Definition

\begin{tabular}{|c|c|c|}
\hline Abkürzung & Bedeutung & Definition \\
\hline TIB & $\begin{array}{l}\text { Time in bed } \\
\text { Bettzeit }\end{array}$ & $\begin{array}{l}\text { Zeit zwischen Zubettgehen (Licht aus) und Wecken } \\
\text { (Licht an) in Minuten }\end{array}$ \\
\hline $\mathrm{SPT}$ & $\begin{array}{l}\text { Sleep period time } \\
\text { Schlafperioden- } \\
\text { dauer }\end{array}$ & $\begin{array}{l}\text { Zeit zwischen Schlafbeginn (Beginn St. } 2 \text { oder eines } \\
\text { anderen Schlafstadiums außer St. 1) und der } \\
\text { letzten Epoche Schlaf }\end{array}$ \\
\hline TST & $\begin{array}{l}\text { Total sleep time } \\
\text { Gesamtschlafzeit }\end{array}$ & $\begin{array}{l}\text { Schlafperiodendauer abzüglich aller Wachzeiten } \\
\text { und Pausen; MT wird dabei als Schlafzeit gewertet }\end{array}$ \\
\hline MT & Movement time & Bewegungszeit \\
\hline $\mathrm{SE}$ & $\begin{array}{l}\text { Sleep efficiency } \\
\text { Schlafeffizienz }\end{array}$ & $\begin{array}{l}\text { Verhältnis von TST gegenüber TIB, angegeben in } \\
\text { Prozent }\end{array}$ \\
\hline SL & $\begin{array}{l}\text { Sleep latency } \\
\text { Schlaflatenz }\end{array}$ & $\begin{array}{l}\text { Zeit von Licht aus bis zur 1. Epoche eines } \\
\text { definierten Schlafstadiums }\end{array}$ \\
\hline SL-1 & Schlaflatenz-1 & Zeit von Licht aus bis zur 1. Epoche St. 1 \\
\hline SL-2 & Schlaflatenz-2 & Zeit von Licht aus bis zur 1. Epoche St. 2 \\
\hline SWS-L & Tiefschlaf-Latenz & Zeit ab Beginn St. 2 bis zur 1. Epoche St. 3 oder 4 \\
\hline REM-L & REM-Latenz & Zeit ab Beginn St. 2 bis zur 1. REM-Episode \\
\hline REM-Dichte & & $\begin{array}{l}\text { Augenbewegungsdichte bezogen auf alle } \\
\text { REM-Perioden }\end{array}$ \\
\hline REM-Dichte-1 & & $\begin{array}{l}\text { Augenbewegungsdichte bezogen auf die } 1 . \\
\text { REM-Periode }\end{array}$ \\
\hline Anz. Aufwachv. & $\begin{array}{l}\text { Anzahl Aufwach- } \\
\text { vorgänge }\end{array}$ & $\begin{array}{l}\text { Auftreten mindestens einer Epoche St. Wach } \\
\text { während SPT }\end{array}$ \\
\hline PLMS & $\begin{array}{l}\text { Periodic leg move- } \\
\text { ments in sleep }\end{array}$ & Anzahl periodischer Beinbewegungen im Schlaf \\
\hline $\mathrm{W}, \% \mathrm{SPT}$ & & Anteil St. Wach bezogen auf SPT in Prozent \\
\hline St. 1, \% SPT & & Anteil St. 1 bezogen auf SPT in Prozent \\
\hline St. $2, \%$ SPT & & Anteil St. 2 bezogen auf SPT in Prozent \\
\hline St. $3, \%$ SPT & & Anteil St. 3 bezogen auf SPT in Prozent \\
\hline St. $4, \%$ SPT & & Anteil St. 4 bezogen auf SPT in Prozent \\
\hline REM, \% SPT & & Anteil REM bezogen auf SPT in Prozent \\
\hline SWS, \% SPT & & Anteil SWS bezogen auf SPT in Prozent \\
\hline
\end{tabular}


dem Schlaf (GES), die psychische Ausgeglichenheit am Abend (PSYAA), die Psychische Erschöpftheit am Abend (PSYEA) und psychosomatische Symptome in der Schlafphase (PSS) erfasst.

Ferner wurde den Studienteilnehmern die VIS-M vorgelegt. Dieser Fragebogen besteht aus 12 Punkten, darunter zwei visuelle Analogskalen. Die Auswertung erfolgte nach festem Schema. Bei den visuellen Analogskalen handelte es sich jeweils um eine $100 \mathrm{~mm}$ lange Linie, deren Endpunkte extreme Zustände darstellten. Die subjektive Empfindung wurde durch ein Kreuz auf der Linie markiert und in Millimeter Entfernung vom Linienbeginn angegeben. Damit wurden die Müdigkeit am Morgen (Wie fühlen Sie sich jetzt am Morgen nach dem Aufstehen? Wundervoll frisch und energiegeladen $\leftrightarrow--\rightarrow$ schrecklich müde und lustlos) und die Schlafqualität der voran gegangenen Nacht (Wie haben Sie heute Nacht geschlafen? Sehr gute Nacht $\leftrightarrow---\rightarrow$ sehr schlechte Nacht) erfasst. Zusätzlich wurden noch die subjektive Schlaflatenz (min), die erinnerte Anzahl der Aufwachvorgänge und die subjektive Schlafdauer (min) dokumentiert.

\subsubsection{Kortisolbestimmung im Urin}

Die Kortisolbestimmung wurde nach erprobten Standardverfahren im Neurobiologischen Labor der Abteilung für Psychiatrie und Psychotherapie der Universität Göttingen durchgeführt. Wie in anderen Studien zuvor (Cohrs et al., 2004a; Hajak et al., 1997) wurde die Kortisolkonzentration nach Extraktion aus dem Urin mittels Dichlormethan durch einen Radioimmunoassay bestimmt. Dabei lag die Interassay-Varianz bei $10 \%$ und die untere Nachweisgrenze bei 3,75 pg/100 $\mu \mathrm{l}$. Über die gemessene Kortisolkonzentration wurde die Kortisolausscheidung in ng als Produkt von Urinvolumen [ml] und Kortisolkonzentration [ng/ml] bestimmt. Zur besseren Übersicht wurde die Kortisolausscheidung von ng in $\mu \mathrm{g}$ umgerechnet und angegeben.

\subsection{Statistik}

Mit Hilfe des Programms STATISTICA 6.1 ${ }^{\circledR}$ (StatSoft) erfolgte die statistische Auswertung. Die Ergebnisse der Polysomnographie, der Fragebögen und der Kortisolbestimmung wurden zunächst deskriptiv als Mittelwerte (MW) mit Standardabweichung (Standard deviation, SD) dargestellt. Varianzanalysen (Analysis of variance, ANOVA) mit Messwiederholung wurden für jeden einzelnen Parameter durchgeführt, um den Medikationseffekt (Ziprasidon vs. Plazebo), den Stresseffekt (ungestörter vs. gestörter Schlaf) und den Interaktionseffekt (Interaktion zwischen Medikation und akustischem Stress) zu erfassen. Waren die F-Werte signifikant, wurden Post-Hoc 
t-Tests angewandt, um, sofern möglich, statistisch signifikante Unterschiede darzustellen. Das Signifikanzniveau wurde auf $p<0,05$ festgesetzt. Signifikante Ergebnisse der polysomnographischen Parameter nach ANOVA wurden einer $\alpha$-Korrektur unterzogen und als „Overall“-Signifikanz $(P)$ unter Benutzung der Methode nach Cross und Chaffin (1982) ausgedrückt (Bortz, 2005). 


\section{Kapitel 4}

\section{Ergebnisse}

\subsection{Demographische Daten}

Für die Studie wurden insgesamt 16 gesunde männliche Probanden mit einem Durchschnittsalter von 26,7 \pm 4,2 Jahren in einem Altersbereich von 20-35 Jahren rekrutiert.

Die Probandengruppe setzte sich aus Studenten und Doktoranden der Georg-AugustUniversität zusammen. Von den 16 zunächst in die Studie eingeschlossenen Probanden konnten nur die Daten von 11 bzw. 12 Probanden verwendet werden. Dies geschah aus folgenden Gründen: Bei einem Probanden kam es nach erstmaliger Einnahme von $40 \mathrm{mg}$ Ziprasidon zu einer Synkope. Höchstwahrscheinlich wurde diese durch eine orthostatische Hypotonie hervorgerufen. Ein anderer zog seine Einwilligung zur Teilnahme an der Studie während der ersten Nacht mit akustischem Stress bei vorheriger Gabe von Plazebo zurück. Bei zwei Individuen traten während der ersten Nacht technische Probleme bei der Ableitung im Rahmen der Polysomnographie auf, so dass nur unvollständige Datensätze vorliegen. Die genannten vier Probanden wurden komplett von der Studienauswertung ausgeschlossen. Die Daten der verbleibenden 12 Probanden konnten polysomnographisch und testpsychologisch ausgewertet werden. Von den 12 Probanden konnte ein Proband in einem der geforderten Zeiträume (unter Plazebo) nicht urinieren, so dass bei der Auswertung der Kortisolexkretion lediglich die Daten von 11 Probanden berücksichtigt wurden.

\subsection{Erfasste Schlafparameter}

\subsubsection{Objektive Schlafparameter}

In den Tabellen A.1-A.3 im Anhang ist eine Gesamtübersicht der pro Proband gemessenen Schlafparameter dargestellt. Tabelle 4.1 zeigt die polysomnographisch ermit- 
Tabelle 4.1: Werte der polysomnographisch erfassten Schlafparameter unter Angabe des Medikations-, Stress- und Interaktionseffekts, MW (SD) und ANOVA $(F(d f=1,11), p<0,05)$, signifikante $p$-Werte sind fett dargestellt

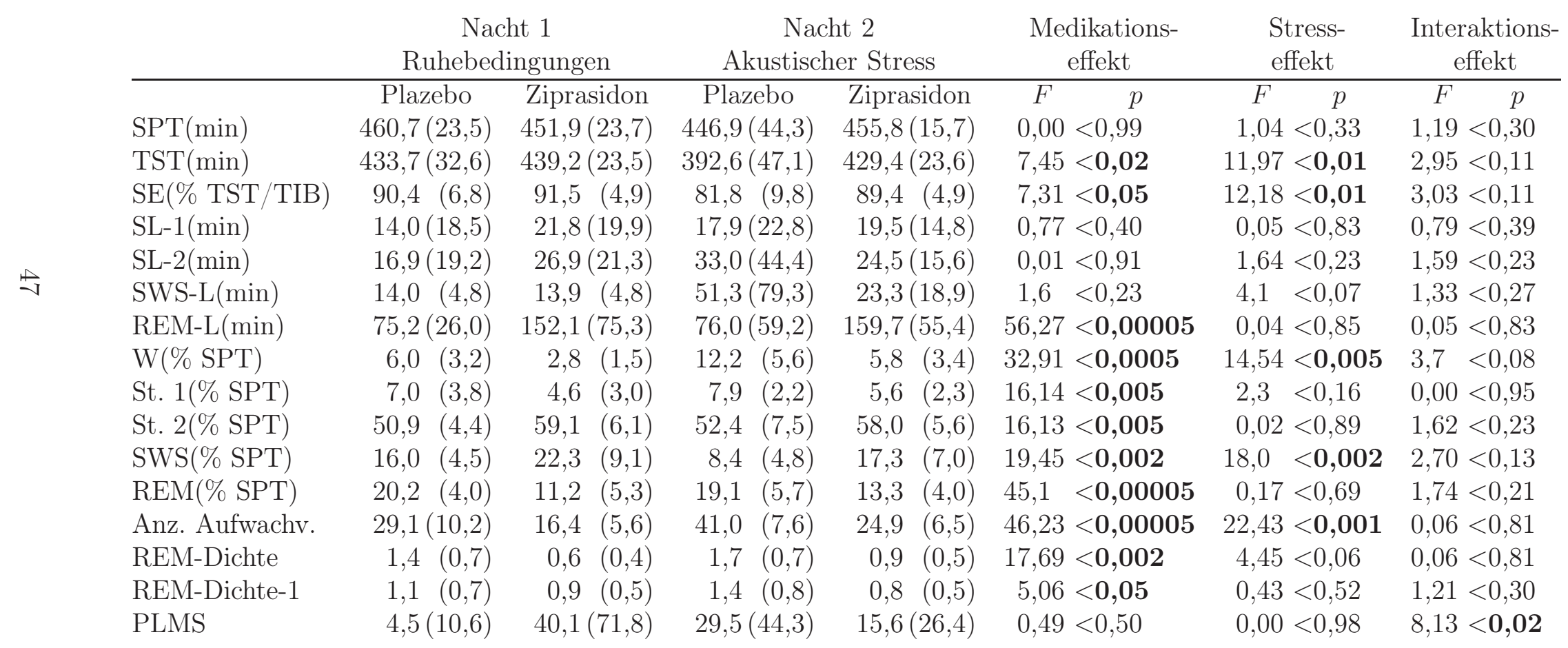



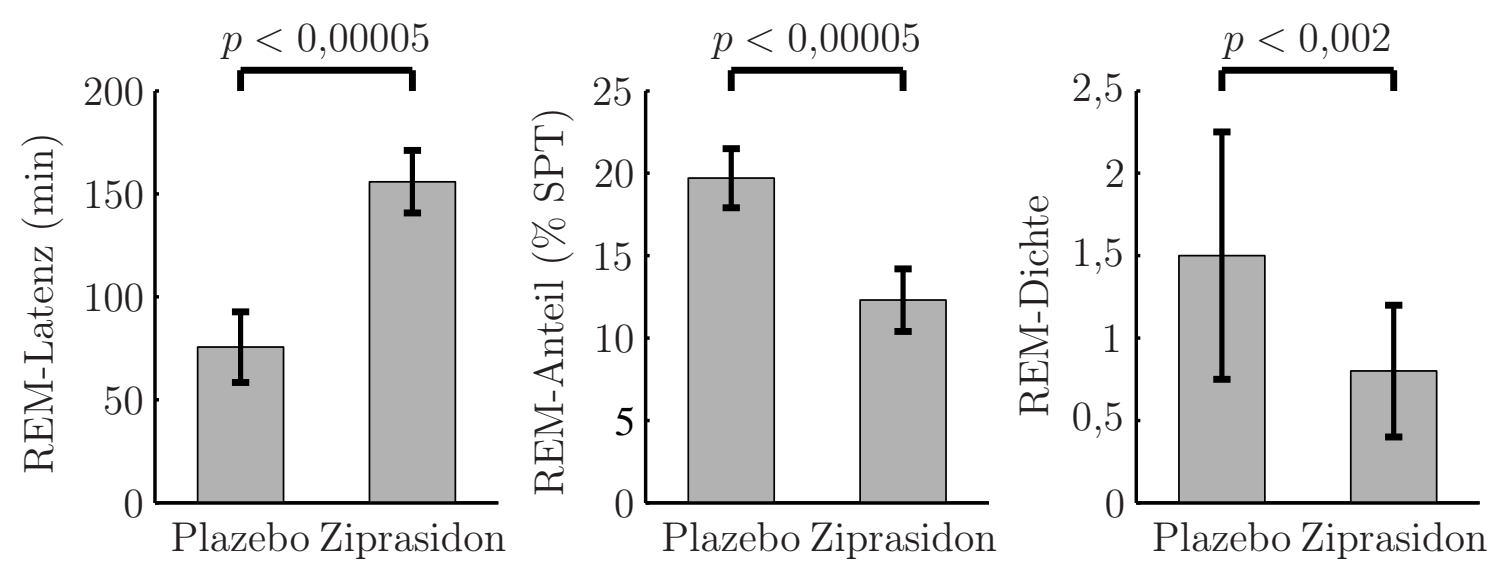

Abbildung 4.1: Der Effekt von $40 \mathrm{mg}$ Ziprasidon auf den REM-Schlaf, Summe der REM-Parameter-Mittelwerte von Nacht 1 und 2 unter Plazebo bzw. Ziprasidon

telten Schlafparameter unter allen Bedingungen. Die Ergebnisse sind als Mittelwerte (MW) mit Standardabweichung (SD) aufgeführt. Daneben zeigt die Tabelle das Ergebnis der statistischen Auswertung mittels Varianzanalyse mit Messwiederholung (Analysis of variance, ANOVA). Berechnet wurde der Medikations-, Stress- und Interaktionseffekt. Der Medikationseffekt (Ziprasidon vs. Plazebo) beleuchtet die Auswirkungen Ziprasidons auf den Schlaf im Vergleich zu Plazebo, während der Stresseffekt (Nacht 1 vs. Nacht 2) ungestörten mit gestörtem Schlaf vergleicht. Zusätzlich wurde der Interaktionseffekt erfasst, der die Wechselwirkung zwischen Medikation und akustischem Stress widerspiegelt.

Hinsichtlich des Medikationseffekts ergab sich folgendes: Im Vergleich zu Plazebo erhöhte sich unter Ziprasidon die Schlafeffizienz (SE=TST/TIB), die Gesamtschlafzeit (TST), die REM-Latenz (REM-L) sowie der Anteil des Stadiums 2 und des Tiefschlafs an der Schlafperiodendauer (Sleep period time, SPT) signifikant. Der im Stadium Wach verbrachte Anteil, der Anteil an Stadium 1, die Anzahl der Aufwachvorgänge (Anz. Aufwachv.), der REM-Anteil sowie die gesamte REM-Dichte über alle REM-Perioden als auch die REM-Dichte der ersten REM-Periode (REM-Dichte-1) verminderten sich. Die „Overall“-Signifikanz (11 von 16 Tests) lag bei $P<0,0001$. Abbildung 4.1 veranschaulicht den Effekt von Ziprasidon auf den REM-Schlaf graphisch. Die Abbildung zeigt die Mittelwerte der REM-Parameter von Nacht 1 und Nacht 2 unter Plazebo bzw. unter Ziprasidon. Im Anhang finden sich in Tabelle A.4 die Werte hierzu.

Bei der Berechnung des Stresseffekts zeigten sich ebenfalls einige Parameter signifikant verändert. Im Vergleich zu ungestörtem Schlaf verringerten sich unter akustisch gestörtem Schlaf die TST, die Schlafeffizienz und der Tiefschlaf-Anteil. Der im Sta- 
dium Wach verbrachte Schlaf-Anteil und die Anzahl der Aufwachvorgänge erhöhten sich. Bezüglich der REM-Dichte ließ sich nur eine Tendenz $(p<0,06)$ zur Vergrößerung der REM-Dichte feststellen. Die „Overall“-Signifikanz (fünf von 16 Tests) betrug $P<0,001$.

Die Bestimmung des Interaktionseffekts zwischen Medikation (Ziprasidon vs. Plazebo) und Stress (Nacht 1 vs. Nacht 2) wies bis auf die periodischen Beinbewegungen im Schlaf (PLMS) keine signifikanten Veränderungen auf. Bezüglich des Interaktionseffekts ergab sich eine „Overall“-Signifikanz (einer von 16 Tests) von $P<0,5$.

\subsubsection{Subjektive Schlafparameter}

In Analogie zu den mittels Polysomnographie objektiv erfassten Schlafparametern werden in Tabelle 4.2 die subjektiven Schlafparameter, die durch die Fragebögen ermittelt wurden, als Mittelwerte (MW) mit Standardabweichung (SD) aufgezeigt. Weiter zeigt auch diese Tabelle die $F$ - und $p$-Werte der verschiedenen Effekte nach ANOVA.

Nach Auswertung des Schlaffragebogen-A (SF-A) zeigte sich als Medikationseffekt eine signifikant verbesserte Schlafqualität und eine verminderte Anzahl psychosomatischer Symptome in der Schlafphase (PSS). Nach der „Visuellen Analogskala morgens" (VIS-M) ergab sich unter Ziprasidon eine signifikant verminderte Anzahl der erinnerten Aufwachvorgänge und eine erhöhte subjektive Schlafdauer. Die „Overall“Signifikanz (vier von zehn Tests) betrug $P<0,005$.

Unter akustischem Stress (Stresseffekt) nahmen die Schlafqualität (SQ), das Gefühl des Erholtseins nach dem Schlaf (GES) und die psychische Erschöpftheit am Abend (PSYEA) ab. Dagegen stiegen die psychosomatischen Symptome in der Schlafphase (PSS) an. Die Auswertung der VIS-M ergab ebenfalls signifikante Unterschiede. Die Schlafqualität und die subjektive Schlafdauer nahmen ab, während die subjektive Schlaflatenz und die Anzahl der Aufwachvorgänge anstiegen. Die Berechnung der „Overall"-Signifikanz (acht von zehn Tests) ergab $P<0,00005$.

Bei der Betrachtung des Interaktionseffekts zeigte sich nur ein signifikanter Unterschied bei dem Gefühl des Erholtseins nach dem Schlaf (GES) des SF-A und bei der Müdigkeit am Morgen der VIS-M. Die „Overall"-Signifikanz (zwei von zehn Tests) betrug $P<0,09$. Sie stellt somit also nur eine Tendenz dar. Anschließende t-Tests legten dar, dass die Studienteilnehmer nach Gabe von Ziprasidon in Nacht 1 unter Ruhebedingungen ein geringeres Gefühl des Erholtseins zeigten als bei Gabe von Plazebo. In Nacht 2 unter akustischem Stress ließ sich kein signifikanter Einfluss von Ziprasidon feststellen. Eine Tendenz zu vermehrter Müdigkeit am Morgen zeigte sich unter Ziprasidon nach Nacht 1, nicht aber nach Nacht 2. 
Tabelle 4.2: Subjektive Schlafparameter unter Angabe des Medikations-, Stress- und Interaktionseffekts, MW (SD) und ANOVA $(F(d f=1,11), p<0,05)$, signifikante $p$-Werte sind fett dargestellt

\begin{tabular}{|c|c|c|c|c|c|c|c|c|}
\hline & \multicolumn{2}{|c|}{$\begin{array}{c}\text { Nacht } 1 \\
\text { Ruhebedingungen } \\
\end{array}$} & \multicolumn{2}{|c|}{$\begin{array}{c}\text { Nacht } 2 \\
\text { Akustischer Stress } \\
\end{array}$} & $\begin{array}{c}\text { Medikations- } \\
\text { effekt }\end{array}$ & \multicolumn{2}{|c|}{$\begin{array}{l}\text { Stress- } \\
\text { effekt }\end{array}$} & $\begin{array}{c}\text { Interaktions- } \\
\text { effekt }\end{array}$ \\
\hline SF-A & Plazebo & Ziprasidon & Plazebo & Ziprasidon & $F \quad p$ & $F$ & $p$ & $\begin{array}{ll}F & p\end{array}$ \\
\hline Schlafqualität & $3,7 \quad(0,5)$ & $3,9 \quad(0,5)$ & $2,0 \quad(0,6)$ & $2,5 \quad(0,4)$ & $10,78<\mathbf{0 , 0 1}$ & 64,2 & $<0,00001$ & $1,53<0,24$ \\
\hline GES & $3,4 \quad(0,6)$ & $3,0 \quad(0,6)$ & $2,8 \quad(0,4)$ & $3,0 \quad(0,6)$ & $2,63<0,13$ & 6,11 & $<<0,05$ & $15,07<\mathbf{0 , 0 0 5}$ \\
\hline PSYAA & $3,6 \quad(0,7)$ & $3,8 \quad(0,5)$ & $3,6 \quad(0,3)$ & $3,4 \quad(0,5)$ & $0,0<0,95$ & 3,43 & $3<0,09$ & $1,65<0,23$ \\
\hline PSYEA & $2,8 \quad(0,6)$ & $2,7 \quad(0,3)$ & $2,3 \quad(0,4)$ & $2,3 \quad(0,4)$ & $0,47<0,51$ & 12,5 & $<0,005$ & $0,16<0,70$ \\
\hline PSS & $1,5 \quad(0,4)$ & $1,2 \quad(0,2)$ & $2,0 \quad(0,5)$ & $1,7 \quad(0,3)$ & $6,75<\mathbf{0 , 0 5}$ & 27,45 & $5<\mathbf{0 , 0 0 0 5}$ & $0,23<0,64$ \\
\hline VIS-M & & & & & & & & \\
\hline Müdigkeit am Morgen & $45,3(18,7)$ & $54,8(15,1)$ & $56,8(14,9)$ & $49,9(18,7)$ & $0,13<0,73$ & 0,46 & $<<0,51$ & $5,7<\mathbf{0 , 0 5}$ \\
\hline Schlafqualität & $59,7(16,0)$ & $58,3(17,0)$ & $26,9(13,1)$ & $39,3(15,6)$ & $1,12<0,31$ & 49,84 & $4<\mathbf{0 , 0 0 0 1}$ & $2,72<0,13$ \\
\hline Subj. Schlaflatenz & $25,8(22,4)$ & $24,6(20,4)$ & $40,0(23,8)$ & $31,3(14,5)$ & $1,42<0,26$ & 7,1 & $<0,05$ & $0,74<0,41$ \\
\hline Anz. Aufwachv. & $1,4(1,1)$ & $0,9 \quad(0,9)$ & $5,0 \quad(3,2)$ & $4,0 \quad(2,4)$ & $6,91<\mathbf{0 , 0 5}$ & 18,30 & $<0,005$ & $0,65<0,44$ \\
\hline Subj. Schlafdauer & $449,2(27,9)$ & $455,0(27,5)$ & $375,4(76,0)$ & $423,6(34,4)$ & $6,18<\mathbf{0 , 0 5}$ & 12,88 & $<<0,005$ & $4,02<0,07$ \\
\hline
\end{tabular}




\subsection{Kortisolexkretion}

Die Menge der Kortisolausscheidung bezogen auf jeden einzelnen Probanden unter Angabe der dazugehörigen Mittelwerte und Standardabweichungen ist im Anhang in Tabelle A.5 ausführlich dargestellt.

Die Tabelle 4.3 zeigt die Kortisolkonzentration und Kortisolmenge des Urins unter allen Bedingungen und zu den verschiedenen Sammelzeiträumen als Mittelwert (MW) und Standardabweichung (SD). Daneben sind die Ergebnisse der statistischen Auswertung mittels ANOVA, also Medikations-, Stress- und Interaktionseffekt, aufgezeigt.

Im Sammelzeitraum 19-23 Uhr waren keine signifikanten Medikations-, Stressoder Interaktionseffekte hinsichtlich der Menge der Kortisolexkretion zu beobachten. Dies bedeutete unter anderem, dass keine Übertragungseffekte der Medikationsgabe am vorherigen Abend auf den darauf folgenden Abend auftraten.

In der Sammelperiode von 23-7 Uhr ließs sich ein signifikanter Medikationseffekt mit $p<0,0002$, ein Stresseffekt mit $p<0,02$ und ein Interaktionseffekt mit $p<0,02$ feststellen. Hinsichtlich des Medikationseffekts in Nacht 1, also unter Ruhebedingungen, zeigte sich unter Ziprasidon eine signifikant geringere Kortisolexkretion als unter Plazebo. Ziprasidon erniedrigte die ausgeschiedene Kortisolmenge um 4,9 $\mu \mathrm{g}$ (SD 2,4 $\mu \mathrm{g}$ ) im Vergleich zu Plazebo. In Nacht 2 unter akustischem Stress war der Medikationseffekt noch deutlicher, hier verringerte Ziprasidon die mittlere Kortisolmenge sogar um 10,8 $\mu \mathrm{g}$ (SD 7,5 $\mu \mathrm{g}$ ). Die Störung des Schlafs führte unter Plazebo zu einem Kortisolanstieg (Stresseffekt). Die weitere Analyse des Interaktionseffekts zeigte, dass dieser beobachtete Kortisolanstieg nur unter Plazebo, nicht jedoch in dem Maße unter Ziprasidon auftrat.

Während der morgendlichen Sammelperiode von 7-11 Uhr konnte ein signifikanter Medikationseffekt $(p<0,02)$ ermittelt werden. Es zeigte sich eine signifikant herabgesetzte Kortisolausscheidung unter Ziprasidon im Vergleich zu Plazebo. Unter Ruhebedingungen war die durchschnittliche Kortisolexkretion unter Ziprasidon um $5,8 \mu \mathrm{g}(12,8 \mu \mathrm{g})$ geringer als unter Plazebo. Unter akustischem Stress (Nacht 2) war die mittlere Kortisolausscheidung unter Ziprasidon um 12,1 $\mu \mathrm{g}(13,8 \mu \mathrm{g})$ geringer als unter Plazebo. Keine signifikanten Änderungen ergaben sich hinsichtlich des Stressund Interaktionseffekts.

Graphik 4.2 veranschaulicht die Senkung der Kortisolexkretion unter Ziprasidon. Dargestellt sind die Mittelwerte von Nacht 1 und Nacht 2 unter Plazebo bzw. Ziprasidon. Da nur in den Sammelperioden 23-7 und 7-11 Uhr, nicht aber in der Sammelperiode 7-23 Uhr, signifikante Effekte auftraten, sind nur die erstgenannten abgebildet. Die Werte finden sich in Tabelle A.6 des Anhangs. 
Tabelle 4.3: Kortisolkonzentration und Kortisolmenge unter Angabe des Medikations-, Stress- und Interaktionseffekts, MW (SD) und ANOVA $(F(d f=1,11), p<0,05)$, signifikante $p$-Werte sind fett dargestellt

Nacht $1 \quad$ Nacht $2 \quad$ Medikations- Stress- Interaktions-

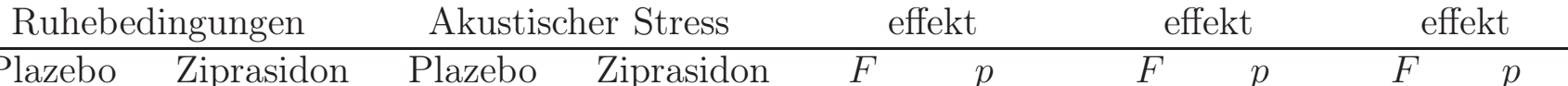

\section{Kortisolmenge / $\boldsymbol{\mu g}$}

19-23.00 h 6,0

23-07:00 h $14,3 \quad(6,5)$

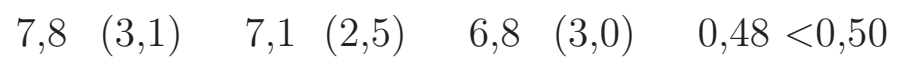

$0,02<0,90 \quad 1,32<0,28$

07-11:00 h $23,8(15,3)$

$\begin{array}{llllllll}9,5 & (5,9) & 22,6 & (9,7) & 11,8 & (5,5) & 36,31<\mathbf{0 , 0 0 0 2}\end{array}$

$8,27<\mathbf{0 , 0 2}$

$7,69<\mathbf{0 , 0 2}$

Kortisolkonzentration $/ \mathrm{ng} / \mathrm{ml}$

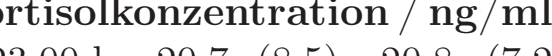

19-23:00 h $20,7 \quad(8,5) \quad 20,8 \quad(7,2)$

23-07:00 h $28,2(12,8) \quad 15,4 \quad(6,2)$

07-11:00 h

$71,8(23,1)$

$92,2(44,1)$

$\begin{array}{lllr}18,4(8,2) & 18,8 & (9,5) & 0,03<0,88 \\ 40,1(12,8) & 18,9 & (9,0) & 28,36<0,0005\end{array}$

$2,27<0,16 \quad 0,03<0,86$

$75,1(29,6)$

$72,0(35,2)$

$0,46<0,51$

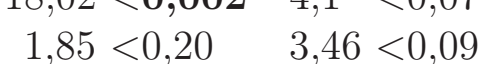



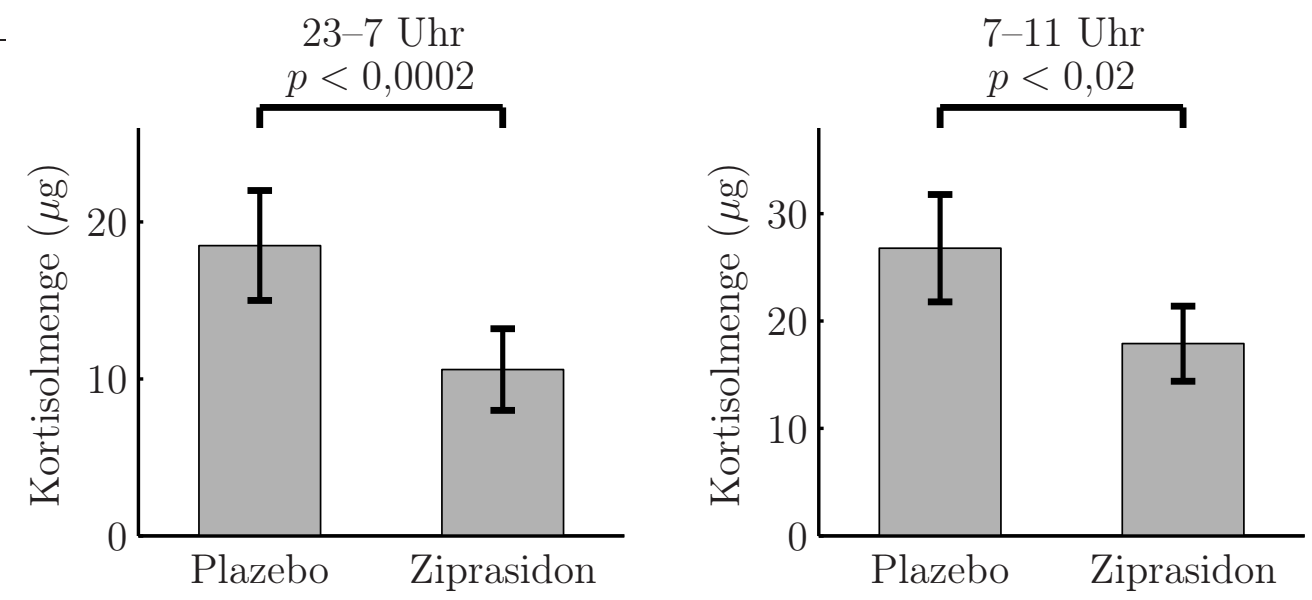

Abbildung 4.2: Der Effekt von $40 \mathrm{mg}$ Ziprasidon auf die Kortisolexkretion, Summe der Mittelwerte von Nacht 1 und 2 unter Plazebo bzw. Ziprasidon 


\section{Kapitel 5}

\section{Diskussion}

Im ersten Abschnitt dieses Kapitels wird zunächst auf den Schlaf eingegangen, im zweiten auf die Kortisolexkretion. Die in Kapitel 4 dargestellten Ergebnisse werden im Hinblick auf die vorhandene Literatur diskutiert. Am Ende dieses Kapitels wird ein Ausblick auf zukünftige Forschungsansätze gegeben.

\subsection{Schlaf}

Wie in Abschnitt 2.2.4 beschrieben, geht eine Schizophrenie in den meisten Fällen mit Schlafstörungen einher. Diese spiegeln sich polysomnographisch vor allem in einer verminderten Schlafkontinuität mit reduzierter Gesamtschlafzeit und Schlafeffizienz wider. Mit höherer Variabilität zeigen sich zudem eine Verminderung des TiefschlafAnteils und der REM-Latenz sowie eine Erhöhung des REM-Anteils und der REMDichte.

Darüber hinaus zeigen insbesondere das Tiefschlaf-Defizit und die verkürzte REMLatenz eine Assoziation sowohl mit schlechterem Krankheitsverlauf als auch mit dem Ausmaß der Negativsymptomatik, worauf in Abschnitt 2.2.6 eingegangen wurde.

Auch wenn die eigentliche Funktion des Schlafs noch nicht vollständig geklärt ist, kann man zeigen, dass bestimmte kognitive Leistungen an spezifische Schlafstadien gebunden sind (vgl. Abschnitt 2.2.2).

Die atypischen Antipsychotika kennzeichnen sich unter anderem durch ihre Wirksamkeit auf Negativsymptome (Benkert und Hippius, 2003; Kane, 2003; Kapur und Mamo, 2003; Möller, 2003). Hierzu gehört auch eine Verbesserung kognitiver Funktionen bei Schizophrenen. Ein Zusammenhang mit ihrer Wirkung auf den Schlaf liegt somit nahe.

Die Datenlage zur Wirkung klassischer und atypischer Antipsychotika auf den Schlaf schizophrener Patienten und gesunder Personen ist begrenzt und die durchgeführten 
Studien sind häufig methodisch unbefriedigend konzipiert. Eine ausführliche Zusammenfassung der Auswirkungen von Antipsychotika auf den Schlaf schizophrener wie gesunder Personen wurde in Abschnitt 2.3 dargelegt. Anhand der vorhandenen Literatur ergibt sich folgendes: Klassische wie atypische Antipsychotika verbessern bei Schizophrenen die Schlafkontinuität. Nicht so einheitlich zeigen sich die Wirkungen dagegen bei Gesunden, bei diesen erhöht lediglich die Gabe der meisten atypischen Antipsychotika die Schlafkontinuität. Aus letzterem lässt sich unter anderem folgern, dass die bei schizophrenen Patienten beobachteten Veränderungen des Schlafs unter klassischen Antipsychotika nicht einen direkten Medikamenteneffekt darstellen, sondern vielmehr auch als Folge einer gebesserten Symptomatik zu interpretieren sind. Um direkte Medikamenteneffekte untersuchen zu können, sind Studien an gesunden Probanden nötig.

Viele Fragen bezüglich der Wirkung atypischer Antipsychotika auf den Schlaf sind noch offen. Die Auswirkungen des atypischen Antipsychotikums Ziprasidon sind bisher in keiner Studie polysomnographisch untersucht worden.

\subsubsection{Einfluss von Ziprasidon auf den Schlaf}

Im Rahmen dieser Arbeit wurden erstmals die Auswirkungen einer mittleren Dosis Ziprasidon auf den ungestörten und akustisch gestörten Schlaf bei gesunden Probanden untersucht.

Die akustische Störung des Schlafs in Form einer nächtlichen Exposition mit standardisierten akustischen Reizen (vgl. Abschnitt 3.5.2) führte bei den gesunden Probanden zu einer Verminderung der Schlafkontinuität, ausgedrückt durch eine Reduktion der Gesamtschlafzeit und Schlafeffizienz sowie durch vermehrte Aufwachvorgänge, und zu einer Abnahme des Tiefschlaf-Anteils.

Diese Schlafparameter zeigten sich somit in der gleichen Richtung verändert wie sie bei unbehandelten Schizophrenen (siehe oben) oder Insomnikern (Hajak et al., 2001) im Rahmen der Krankheit vorkommen. Es konnte auf diese Weise ein InsomnieModell erfolgreich etabliert werden, das Schlafstörungen schizophrener Patienten simuliert.

Ziprasidon zeigte im Rahmen dieser doppel-blinden, randomisierten Studie mit crossover Design eine deutliche schlafkonsolidierende Wirkung. Dies spiegelte sich in einer Reduktion der Anzahl von Aufwachvorgängen sowie in der Zunahme von Gesamtschlafzeit und Schlafeffizienz wider. Ferner waren unter Medikation das Schlafstadium 2 und der Tiefschlaf-Anteil vergrößert, während sich der Anteil an Stadium 1 verkürzte. Besonders ausgeprägt wurde allerdings der REM-Schlaf beeinflusst, sowohl tonische als auch phasische Aspekte des REM-Schlafs wurden unterdrückt. Es zeigte 
Tabelle 5.1: Die Auswirkungen atypischer Antipsychotika auf den Schlaf

\begin{tabular}{|c|c|c|c|c|c|c|c|c|c|c|}
\hline \multirow[b]{2}{*}{ Schlafparameter } & \multicolumn{2}{|c|}{ Clozapin } & \multicolumn{2}{|c|}{ Olanzapin } & \multicolumn{2}{|c|}{ Quetiapin } & \multicolumn{2}{|c|}{ Risperidon } & \multicolumn{2}{|c|}{ Ziprasidon } \\
\hline & Sch & Ges & Sch & Ges & Sch & Ges & Sch & Ges & Sch & Ges \\
\hline Schlafkontinuität & $\uparrow$ & $\uparrow / \leftrightarrow$ & $\uparrow$ & $\uparrow$ & & $\uparrow$ & $\leftrightarrow$ & $\leftrightarrow$ & & $\uparrow$ \\
\hline St. 1 & $\downarrow / \leftrightarrow$ & $\downarrow / \leftrightarrow$ & $\downarrow$ & $\downarrow$ & & $\leftrightarrow$ & $\leftrightarrow$ & $\leftrightarrow$ & & $\downarrow$ \\
\hline St. 2 & $\uparrow$ & $\leftrightarrow$ & $\uparrow$ & $\leftrightarrow$ & 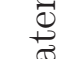 & $\uparrow$ & $\leftrightarrow$ & $\leftrightarrow$ & 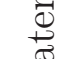 & \\
\hline SWS & $\downarrow / \leftrightarrow$ & $\downarrow / \leftrightarrow$ & $\uparrow$ & $\uparrow$ & $\stackrel{\widetilde{\Omega}}{\rho}$ & $\leftrightarrow$ & $\uparrow$ & $\leftrightarrow$ & $\stackrel{\pi}{\theta}$ & $\uparrow$ \\
\hline REM & $\uparrow / \leftrightarrow$ & $\leftrightarrow$ & $\uparrow / \leftrightarrow$ & $\downarrow / \leftrightarrow$ & $\stackrel{\Xi}{\Xi}$ & $\leftrightarrow$ & $\leftrightarrow$ & $\downarrow$ & $\underset{\Xi}{\nexists}$ & $\downarrow$ \\
\hline REM-L & $\leftrightarrow$ & $\leftrightarrow$ & $\leftrightarrow$ & $\uparrow$ & $\overrightarrow{0}$ & $\leftrightarrow$ & $\leftrightarrow$ & $\leftrightarrow$ & $\overrightarrow{1}$ & $\uparrow$ \\
\hline REM-Dichte & $\uparrow$ & $\uparrow / \leftrightarrow$ & $\uparrow$ & n. b. & & $\leftrightarrow$ & $\leftrightarrow$ & $\leftrightarrow$ & & $\downarrow$ \\
\hline
\end{tabular}

Sch $=$ Schizophrene, Ges $=$ Gesunde, n. b. nicht berichtet,

$\uparrow=$ statistisch signifikant erhöht,

$\downarrow=$ statistisch signifikant erniedrigt,

$\leftrightarrow=$ gleichbleibend oder statistisch nicht signifikant verändert

sich eine ungefähr verdoppelte REM-Latenz und eine deutliche Abnahme des REMAnteils und der REM-Dichte.

Die schlafkonsolidierenden Eigenschaften konnten durch die Auswertung des subjektiven Schlafbefindens anhand der Fragebögen bestätigt werden. Unter Ziprasidon verbesserte sich die empfundene Schlafqualität und die psychosomatischen Symptome in der Schlafphase (PSS) nahmen ab (nach SF-A). Zudem verringerte sich die Anzahl der Aufwachvorgänge und die subjektive Schlafdauer nahm zu (nach VIS-M).

Auf die anderen bisher untersuchten atypischen Antipsychotika wurde im Hinblick auf die vorhandene Literatur bereits in Abschnitt 2.3 ausführlich eingegangen. Für Amisulprid, Aripiprazol und Sertindol liegen allerdings weder bei psychiatrischen Patienten noch bei gesunden Probanden polysomnographische Daten vor. Die Tabelle 5.1 gibt eine Übersicht zu den Auswirkungen der bisher untersuchten atypischen Antipsychotika auf den Schlaf schizophrener und gesunder Personen. In dieser Tabelle ist auch der in dieser Arbeit experimentell ermittelte Einfluss Ziprasidons auf den Schlaf gesunder Probanden eingefügt.

Der Schlaf unter der Einwirkung von Ziprasidon unterschied sich von dem Schlaf unter den anderen bisher untersuchten Atypika. Ziprasidon zeigte als wesentliche Wirkung eine ausgeprägte Suppression des REM-Schlafs, was für andere untersuchte atypische Antipsychotika in dieser Form nicht gezeigt werden konnte. In Analogie zu den anderen atypischen Antipsychotika wies Ziprasidon eine allgemeine schlafkonsolidierende Wirkung auf. Darüber hinaus vergrößerte es den Tiefschlaf-Anteil signifikant. Dies konnte bisher nur für Olanzapin bei gesunden Probanden wie schizophrenen Patienten gezeigt werden (Müller MJ et al., 2004; Salin-Pascual et al., 
Tabelle 5.2: Schlafstörungen bei Schizophrenie und Depression im Vergleich zur Wirkung von Ziprasidon

\begin{tabular}{rccc} 
& Schizophrenie & Depression & Ziprasidon \\
\hline Gesamtschlafzeit & $\downarrow$ & $\downarrow$ & $\uparrow$ \\
Schlaflatenz & $\uparrow$ & $\uparrow$ & $\leftrightarrow$ \\
Aufwachvorgänge & $\uparrow$ & $\uparrow$ & $\downarrow$ \\
Wach-Anteil & $\uparrow$ & $\uparrow$ & $\downarrow$ \\
Schlafeffizienz & $\downarrow$ & $\downarrow$ & $\uparrow$ \\
Tiefschlaf & $\downarrow$ & $\downarrow$ & $\uparrow$ \\
REM-Latenz & $\downarrow$ & $\downarrow$ & $\uparrow$ \\
REM-Anteil & $\leftrightarrow$ & $\uparrow$ & $\downarrow$ \\
REM-Dichte & $\uparrow / \leftrightarrow$ & $\uparrow$ & $\downarrow$ \\
$\uparrow=$ statistisch signifikant erhöht, & & \\
$\downarrow=$ statistisch signifikant erniedrigt, & & \\
$\leftrightarrow=$ gleichbleibend oder statistisch nicht signifikant verändert
\end{tabular}

1999). Auch Risperidon zeigte eine Erhöhung des Tiefschlafs bei Schizophrenen, allerdings ist die Aussagekraft dieser Studie eingeschränkt, da kein Plazebo-Vergleich durchgeführt wurde (Yamashita et al., 2002).

\subsubsection{Vergleich der Wirkung Ziprasidons mit der der Antidepressiva}

Der pathologisch veränderte Schlaf bei depressiven und schizophrenen Patienten wurde bereits in den Abschnitten 2.2.3 und 2.2.4 im Einzelnen beschrieben. Anhand der vorliegenden Literatur wurde die Tabelle 5.2 konzipiert. Sie zeigt eine Übersicht der herausragendsten pathologisch veränderten Schlafparameter bei Schizophrenie und Depression. Zum Vergleich wurde die in dieser Arbeit ermittelte Wirkung von Ziprasidon bei gesunden Probanden mit aufgeführt.

Die auftretenden Schlafstörungen bei Schizophrenie und Depression sind einander sehr ähnlich. Insbesondere eine Störung der Schlafkontinuität mit verminderter Gesamtschlafzeit und Schlafeffizienz, erhöhter Schlaflatenz, vermehrten Aufwachvorgängen sowie einem erhöhten Wach-Anteil liegt bei beiden Erkrankungen vor. Zudem zeigt sich ein reduzierter Tiefschlaf-Anteil bei der Depression. Viele Studien dokumentieren dies auch bei Vorliegen einer Schizophrenie.

Am charakteristischsten unterscheiden sich die beiden Erkrankungen hinsichtlich des REM-Schlafs. Bei der Depression scheint eine noch ausgeprägtere Non-Suppression des REM-Schlafs vorzuliegen. Statistisch signifikant ist dieser Unterschied jedoch 
nicht (Benca et al., 1992). Im Rahmen einer Depression wird vielfach ein erhöhter Wach-Anteil vor allem in den frühen Morgenstunden beschrieben (Benca, 2000), was bei einer Schizophrenie nicht vorzuliegen scheint (Benson und Zarcone, 2000). Einschränkend sollte allerdings angemerkt werden, dass dieser Faktor in Studien an Schizophrenen auch häufig nicht dokumentiert worden ist.

Es fällt auf, dass Ziprasidon insbesondere die Schlafparameter, die bei einer Depression gestört sind, in entgegengesetzter Weise beeinflusst. Im Folgenden soll die Wirkung Ziprasidons mit der der Antidepressiva verglichen werden.

Die verschiedenen Antidepressiva zeigen variable Auswirkungen auf den Schlaf. Ähnlich wie die Antipsychotika bewirken auch die Antidepressiva häufig einen anderen Effekt bei Patienten als bei Gesunden. Dies begründet sich zum Teil auf einer primären Besserung der krankheitsspezifischen Symptome. Erst sekundär kommt es bei Patienten dann zu einer Veränderung des Schlaf-EEGs. Bei Gesunden erfasst man dagegen die primären Arzneimitteleffekte auf den Schlaf.

Im Gegensatz zu der Diversität der Antipsychotika unterdrücken die Antidepressiva jedoch nahezu einheitlich den REM-Schlaf (Gursky und Krahn, 2000; Mayers und Baldwin, 2005; Riemann et al., 2001; Rijnbeek et al., 2003; Sharpley und Cowen, 1995; Winokur et al., 2001). Eine klinische Verbesserung der depressiven Symptome ist nicht zwingend an eine REM-Suppression gebunden, da einige wirksame Antidepressiva den REM-Schlaf nicht unterdrücken, sondern teilweise sogar erhöhen. Hierzu gehören der reversible Monoaminooxidase-A-Inhibitor Moclobemid (Monti JM, 1989) und weitere Antidepressiva wie Mirtazapin (Aslan et al., 2002), Bupropion (Nofzinger et al., 1995) und Nefazodon (Rush et al., 1998; Sharpley et al., 1996). Das trizyklische Antidepressivum Trimipramin zeigt unterschiedliche Effekte auf den REM-Schlaf. Einige Autoren haben bei depressiven Patienten bzw. Insomnie-Patienten unter Trimipramin keine oder nur geringe Effekte auf den REM-Schlaf zeigen können (Mouret et al., 1989; Riemann et al., 2002; Ware et al., 1989). Im Gegensatz hierzu berichten Feuillade et al. (1992) eine deutliche Erhöhung der REM-Latenz bei gesunden und depressiven Personen unter Trimipramin.

Da bei Depressiven die REM-Dichte zumeist charakteristisch erhöht ist (Benca, 2000; Riemann et al., 2001), würde man am ehesten erwarten, dass sich dieser Parameter unter antidepressiver Therapie normalisiert. Dies scheint aber nicht der Fall zu sein. Sowohl das trizyklische Antidepressivum Trimipramin (Buysse et al., 1996; Reynolds et al., 1997) als auch der SSRI Fluoxetin (Armitage et al., 1997; Trivedi et al., 1999) führen bei Depressiven zu einer weiteren Erhöhung der REM-Dichte. Zudem gibt es Hinweise darauf, dass eine andauernde Erhöhung der REM-Dichte gegen einen depressiven Rückfall schützt (Buysse et al., 1996; Winokur et al., 2001). Bei Gesunden zeigt sich unter einer geringen Dosis Imipramin eine Verminderung der REM-Dichte (Okuma et al., 1975), während unter Fluoxetin bei kurz- und längerfristiger Gabe keine signifikante Veränderung der REM-Dichte berichtet wird (Feige et al., 2002). Vor 
dem Hintergrund dieser Ergebnisse und der Tatsache, dass nur in wenigen Studien der Parameter REM-Dichte überhaupt dokumentiert worden ist, fällt eine abschließende Aussage zur Wirkung der Antidepressiva auf die REM-Dichte schwer.

Die Effekte der Antidepressiva auf den Tiefschlaf sind weit weniger einheitlich als ihre Effekte auf den REM-Schlaf (Sharpley und Cowen, 1995). Zusammengefasst zeigt sich jedoch bei den meisten Antidepressiva, mit Ausnahme der SSRI, eine Tendenz zur Erhöhung des Tiefschlafs (Sharpley und Cowen, 1995).

Die Schlafkontinuität wird durch die meisten trizyklischen Antidepressiva erhöht, während sie durch SSRIs und Monoaminooxidasehemmer häufig erniedrigt wird (Sharpley und Cowen, 1995; Winokur et al., 2001).

Am meisten ähneln die unter Ziprasidon beobachteten Schlafveränderungen den Veränderungen, wie sie unter Therapie mit trizyklischen Antidepressiva beschrieben werden. Darüber hinaus reduziert Ziprasidon aber auch die REM-Dichte, wobei derzeit allerdings noch nicht abschließend geklärt werden kann, welche Relevanz dies hat.

Klinisch zeigt Ziprasidon eine antidepressive Wirksamkeit (Barbee et al., 2004; Papakostas et al., 2004), die möglicherweise auch über den Einfluss auf den Schlaf vermittelt wird.

\subsubsection{Interpretation der Wirkung Ziprasidons auf den Schlaf}

Auf die wesentlichen Theorien, die versuchen, die Charakteristika des Schlafs bei Depression und Schizophrenie zu erklären, wurde im Abschnitt 2.2.5 eingegangen.

Im Folgenden soll der Versuch gemacht werden, die besondere Wirkungsweise Ziprasidons auf den Schlaf näher zu beleuchten.

Die Verlängerung der REM-Latenz und die Verminderung des REM-Anteils unter Ziprasidon könnten durch die Hemmung der Wiederaufnahme von Serotonin und Noradrenalin hervorgerufen sein (vgl. Abschnitt 2.1.2). Wie Wilson und Argyropoulos (2005) in ihrem Literatur-Review hervorheben, üben diejenigen Antidepressiva den größten Effekt auf den REM-Schlaf aus, die die Serotonin-Funktion über eine Hemmung der Wiederaufnahme oder des Metabolismus erhöhen. Ziprasidon besitzt eine ähnliche Wirkstärke in der Hemmung der Wiederaufnahme von Serotonin und Noradrenalin, wie Imipramin und Amitriptylin aus der Gruppe der trizyklischen Antidepressiva (Schmidt et al., 2001). Auch Imipramin (Jobert et al., 1999; Kupfer et al., 1979) und Amitriptylin (Gillin et al., 1978; Kerkhofs et al., 1990; Kupfer et al., 1976; Riemann et al., 1990) bewirken bei Gesunden wie bei Depressiven eine REMSuppression. 
Die Interpretation der verminderten REM-Dichte unter Ziprasidon ist schwieriger. Der physiologische Mechanismus der schnellen Augenbewegungen, die den REMSchlaf maßgeblich kennzeichnen, ist nicht vollständig geklärt. Die Studien von Gillin et al. $(1994,1996)$ belegen eine Verminderung der REM-Dichte durch den 5-HT $1 \mathrm{~A}^{-}$ Agonisten Ipsapiron sowohl bei gesunden Probanden als auch bei depressiven Patienten. In gleicher Weise könnte der Schlaf auch unter Ziprasidon beeinflusst worden sein. Wie in Abschnitt 2.1.2 erläutert, besitzt Ziprasidon eine solche 5-HT $\mathrm{H}_{1 \mathrm{~A}}$-agonistische Wirkkomponente.

Möglicherweise führt aber auch der unter Ziprasidon erhöhte Tiefschlaf-Anteil per se zu einer erniedrigten REM-Dichte. Schon Borbély und Wirz-Justice (1982) brachten ein, dass die REM-Dichte invers mit der Menge an Tiefschlaf korreliert. Lucidi et al. (1996) bestätigten dies in einer Studie. Sie stellen fest, dass die Dauer eines Schlafentzugs negativ mit der REM-Dichte und positiv mit der Menge an Tiefschlaf in der darauf folgenden Nacht korreliert (Lucidi et al., 1996). De Gennaro et al. (2000) führten diese Forschungen weiter und zeigten in ihrer Studie eine Korrelation zwischen verminderter REM-Dichte und Tiefschlaf-Rebound nach selektivem Tiefschlaf-Entzug.

Mehrere Studien haben eine Erhöhung des Tiefschlaf-Anteils durch den 5-HT2-Antagonisten Ritanserin festgestellt (Idzikowski et al., 1986; Paiva et al., 1988). Anhand späterer Studien spezifizieren Sharpley et al. (1994, 2000) dies genauer und machen neben einem 5- $\mathrm{HT}_{2 \mathrm{~A}}$ Antagonismus vor allem eine Blockade zentraler 5- $\mathrm{HT}_{2 \mathrm{C}}$-Rezeptoren für die Erhöhung des Tiefschlaf-Anteils verantwortlich. Da auch Ziprasidon ein potenter Antagonist an 5- $\mathrm{HT}_{2 \mathrm{C}}$-Rezeptoren ist, könnte die beobachtete Erhöhung des Tiefschlafs auf diesem Prinzip beruhen.

\subsection{Kortisolexkretion}

Die Kortisolexkretion ist als ein Spiegel des Aktivierungsgrads der HypothalamusHypophysen-Nebennieren-Achse (Hypothalamic-pituitary-adrenal axis, HPA-Achse) zu betrachten.

Wie in Abschnitt 2.4 beschrieben, konnte eine Regulationsstörung der HPA-Achse als ein wesentlicher pathophysiologischer Bestandteil für eine Reihe psychiatrischer Erkrankungen ermittelt werden (Rothschild, 2003).

Zahlreiche Studien deuten darauf hin, dass nicht nur bei der Depression, sondern auch bei der Schizophrenie eine Über-Aktivierung der HPA-Achse eine bedeutende Rolle spielt (vgl. Abschnitt 2.4.1). 
Bei Schizophrenen wird darüber hinaus auch die Ausprägung von Negativsymptomen, depressiven Symptomen, kognitiven Defiziten, internistischen Begleiterkrankungen und in geringerem Umfang auch der Krankheitszustand durch die Aktivität der HPA-Achse beeinflusst (vgl. Abschnitt 2.4.2). Aus diesem Grund ist es von besonderer klinischer Relevanz zu untersuchen, ob diese Faktoren durch eine antipsychotische Therapie positiv beeinflusst werden.

In einer Reihe von Studien konnte man bisher zeigen, dass die Atypika eine bessere Wirkung auf die Negativsymptomatik besitzen als die klassischen Antipsychotika (Möller, 2003).

Darüber hinaus gibt es Belege für die antidepressive Wirksamkeit atypischer Antipsychotika. Olanzapin, Risperidon, Ziprasidon und Aripiprazol weisen eine antidepressive Wirksamkeit bei bipolarer Depression und behandlungsresistenter Depression im Rahmen der Augmentation einer antidepressiven Therapie auf (Barbee et al., 2004; Nemeroff, 2005; Papakostas et al., 2004; Shelton et al., 2001). Für Olanzapin und Quetiapin konnte darüber hinaus ein antidepressiver Effekt bei Verwendung als Monotherapeutikum im Rahmen einer bipolaren Depression gezeigt werden (Calabrese et al., 2005; Tohen et al., 2003). Dies steht im Gegensatz zu den klassischen Antipsychotika, die eher eine depressiogene Wirkung zeigen (Bandelow et al., 1992; Harrow et al., 1994; Müller P et al., 1978).

Studien belegten eine verbesserte kognitive Leistungsfähigkeit nach einem Wechsel zu Ziprasidon, zu Olanzapin oder zu Quetiapin im Vergleich zum vorherigen Therapieregime bzw. zur Therapie mit klassischen Antipsychotika (Cuesta et al., 2001; Harvey et al., 2004b; Velligan et al., 2003). Eine Studie belegt sogar eine verbesserte kognitive Leistungsfähigkeit nach dem Wechsel von konventionellen Antipsychotika, Olanzapin oder Risperidon zu Ziprasidon (Harvey et al., 2004a). Zahlreiche Studien dokumentieren eine Volumenminderung der Hippocampi bei Schizophrenie (Bogerts et al., 1985; Falkai und Bogerts, 1986; Waldo et al., 1994). Dies ist eine Hirn-Formation, die eine große Rolle bei der Gedächtnisfunktion spielt. Es besteht eine Assoziation zwischen erhöhten Kortisolspiegeln, reduziertem Hippocampusvolumen und verminderter Gedächtnisfunktion (Starkman et al., 1992). Derzeit mehren sich die Hinweise, dass die atypischen Antipsychotika möglicherweise die hirnstrukturellen Veränderungen, die im Rahmen der Schizophrenie an sich oder als Folge einer Therapie mit klassischen Antipsychotika auftreten, mildern können (Scherk und Falkai, 2006).

Die Auswirkungen atypischer Antipsychotika auf internistische Begleiterkrankungen werden kontrovers diskutiert. Clozapin, Olanzapin und in geringerem Umfang auch Risperidon üben beispielsweise einen negativen Effekt auf die Glukosetoleranz aus (Newcomer et al., 2002). Aber auch bei bisher unbehandelten Schizophrenen zeigen sich erhöhte Nüchtern-Glukosewerte und eine vermehrte Insulinresistenz im Vergleich zu Gesunden (Ryan et al., 2003). Die Assoziation zwischen antipsychotischer Medikation und späterer Entwicklung eines Diabetes Mellitus wird von Holt und Peveler 
(2006) in einem Übersichtsartikel beleuchtet. Ihren Recherchen nach scheinen Fallstudien und retrospektive pharmako-epidemiologische Studien den Zusammenhang zwischen sowohl konventionellen als auch atypischen Antipsychotika und einem erhöhten Diabetesrisiko zu belegen. Im Gegensatz dazu haben die prospektiv erhobenen Daten sehr unterschiedliche Ergebnisse erbracht und sind damit wenig aufschlussreich (Holt und Peveler, 2006). Einer erhöhten Stressbereitschaft und daraus resultierenden erhöhten Kortisolspiegeln kommt möglicherweise auch eine wichtige Rolle bei der Entstehung einer Schizophrenie zu; darüber hinaus können diese Faktoren insbesondere bei schizophrenen Patienten zu der Entwicklung eines Diabetes Mellitus beitragen (Dinan, 2004).

Thakore et al. (2002) und Ryan et al. (2004) dokumentieren neben erhöhten Kortisolspiegeln einen erhöhten abdominellen Fettgewebsanteil bei Schizophrenen im Vergleich zu gesunden Probanden. Während allerdings der Kortisolspiegel durch die Gabe der atypischen Antipsychotika gesenkt werden kann, zeigte sich der abdominelle Fettgewebsanteil durch die Therapie unbeeinflusst (Ryan et al., 2004). Derzeit lässt sich noch nicht hinreichend klären, ob die internistischen Folgen einer erhöhten Aktivität der HPA-Achse oder die Disposition Schizophrener zu bestimmten internistischen Krankheiten durch Therapie mit atypischen Antipsychotika positiv oder negativ beeinflusst werden.

\subsubsection{Einfluss von Ziprasidon auf die HPA-Achse}

Im Rahmen dieser Arbeit konnte gezeigt werden, dass Ziprasidon die basale nächtliche Kortisolausscheidung im Urin sowohl unter Ruhebedingungen als auch im Rahmen eines Insomnie-Modells unter akustischem Stress statistisch signifikant verminderte. Der Medikationseffekt war dabei noch in der 14 Stunden nach Einnahme des Präparats gewonnenen Urinportion evident.

In Abschnitt 2.5 wurde die Wirkung klassischer und atypischer Antipsychotika auf die HPA-Achse schizophrener Patienten und gesunder Personen anhand der vorhandenen Literatur erläutert. Insbesondere die Studien, die sich mit klassischen Antipsychotika befassen, sind häufig methodologisch unbefriedigend konzipiert und nur wenige Studien haben bisher die Auswirkungen atypischer Antipsychotika auf die HPA-Achse untersucht.

Zusammengefasst lässt sich sagen, dass insbesondere die atypischen Antipsychotika eine Abschwächung der Aktivität der HPA-Achse bewirken. In Analogie zu den Untersuchungen der Wirkung der Antipsychotika auf den Schlaf, ist auch in diesem Fall schwer zu unterscheiden, welche Wirkungen primäre Medikamenteneffekte sind und welche Wirkungen sekundäre Effekte im Rahmen einer gebesserten Symptomatik sind. Die klassischen Antipsychotika lassen allerdings sowohl den Schlaf als auch 
Tabelle 5.3: Die Auswirkungen atypischer Antipsychotika auf den Kortisolspiegel bzw. auf die Kortisolexkretion

\begin{tabular}{lcc|cc|cc|cc|cc|cc} 
& \multicolumn{2}{r|}{ Clozapin } & \multicolumn{2}{|c|}{ Olanzapin } & \multicolumn{2}{|c|}{ Quetiapin } & \multicolumn{2}{|c|}{ Amisulprid } & \multicolumn{2}{|c}{ Risperidon } & \multicolumn{2}{|c}{ Ziprasidon } \\
Sch & Ges & Sch & Ges & Sch & Ges & Sch & Ges & Sch & Ges & Sch & Ges \\
\hline $\begin{array}{l}\text { Kortisol- } \\
\text { spiegel }\end{array}$ & $\downarrow$ & n. b. & $\downarrow$ & $\downarrow$ & n. b. & $\downarrow$ & n. b. & $\leftrightarrow$ & $\downarrow$ & n. b. & n.b. & $\downarrow$
\end{tabular}

Sch $=$ Schizophrene, Ges $=$ Gesunde, n. b. nicht berichtet,

$\uparrow=$ statistisch signifikant erhöht,

$\downarrow=$ statistisch signifikant erniedrigt,

$\leftrightarrow=$ gleichbleibend oder statistisch nicht signifikant verändert

die Kortisolexkretion gesunder Probanden weitgehend unbeeinflusst und zeigen nur bei Patienten Effekte. Dies lässt darauf schließen, dass zumindest ein Teil der Effekte erst sekundär über eine gebesserte Symptomatik zu erklären ist. Dagegen zeigen die atypischen Antipsychotika bei Gesunden wie Schizophrenen vorwiegend homogene Effekte, was darauf hinweisen könnte, dass es sich hierbei vor allem um primäre Arzneimitteleffekte handelt.

Die Tabelle 5.3 zeigt eine Zusammenfassung der Auswirkungen atypischer Antipsychotika auf den Kortisolspiegel schizophrener und gesunder Personen (vgl. Abschnitt 2.5). Für Aripiprazol und Sertindol liegen bisher keine Daten vor.

Wie Ziprasidon besitzen auch Quetiapin und Olanzapin kortisolsenkende Eigenschaften bei gesunden Probanden (Cohrs et al., 2004a, 2006; De Borja Gonçalves Guerra et al., 2005), während für Amisulprid diese nicht gezeigt werden konnten (Wetzel et al., 1994). Für Clozapin (Meltzer, 1989) und Risperidon (Ryan et al., 2004; Zhang et al., 2005) ist bisher nur eine Senkung des Kortisolspiegels bei Schizophrenen gezeigt worden.

\subsubsection{Interpretation der Wirkung Ziprasidons auf die Kortisolexkretion}

Wie in Abschnitt 2.1 beschrieben konnten die Ursachen der Schizophrenie noch nicht vollständig geklärt werden. Nach Modifizierung der ursprünglichen DopaminHypothese aus den 1960ern geht man heute unter anderem von einer kortikal-subkortikalen Dopamin-Imbalance aus, die auch einen Erklärungsansatz für das Auftreten von Positiv- und Negativsymptomen bietet (Abi-Dargham, 2004). Zusätzlich hat man in vielen Studien eine Dysregulation der HPA-Achse bei Schizophrenen nachweisen können. Diese Regulationsstörung bringen Mück-Seler et al. (1999) mit einer Störung im Serotonin-System (5-HT-System) in Verbindung. Darüber hinaus wird ein 
verstärkender Effekt der HPA-Achse auf die Dopamin-Synthese und die DopaminRezeptoren beschrieben (Walker EF und Diforio, 1997). Einiges weist darauf hin, dass auch neurodegenerative Prozesse ursächlich an einer Schizophrenie beteiligt sind. Dieser Prozess kann durch erhöhte Kortisolspiegel möglicherweise noch verstärkt werden.

Insbesondere atypische Antipsychotika üben einen abschwächenden Einfluss auf die Aktivität der HPA-Achse aus. In der Literatur gibt es Hinweise darauf, dass dies vor allem auf dem 5- $\mathrm{HT}_{2}$-Antagonismus dieser Medikamente beruht (Meltzer, 1989; Scheepers et al., 2001).

Verschiedene Studien haben den Einfluss von 5- $\mathrm{HT}_{2}$-Antagonisten auf die Kortisolsekretion bzw. ACTH-Sekretion bei gesunden Probanden untersucht. Die Gabe einer Einzeldosis Ritanserin, einem 5- $\mathrm{HT}_{2 \mathrm{~A}} / 5-\mathrm{HT}_{2 \mathrm{C}}$-Antagonisten, zeigt einen ACTH-senkenden und kortisolsenkenden Effekt (Tepavcević et al., 1994). In einer anderen Studie hat eine längerfristige Ritanserin-Applikation keinen Einfluss auf die basale Hormonsekretion, kann jedoch eine Kortisolssekretion, die durch eine Insulin-induzierte Hypoglykämie hervorgerufen worden ist, abschwächen (Tepavcević et al., 1995). Dagegen hat man für Ketanserin, ebenfalls einem $5-\mathrm{HT}_{2 \mathrm{~A}} / 5-\mathrm{HT}_{2 \mathrm{C}^{-}}$ Antagonisten, aber mit geringerer Affinität zum 5-HT $\mathrm{H}_{2}$-Rezeptor als Ritanserin (Sharpley et al., 1994), keinen direkten Einfluss auf die basale Kortisolsekretion bei gesunden Studienteilnehmern (Gordin et al., 1985; Zoccali et al., 1983) und bei Hunden (Barbieri et al., 1984) zeigen können.

Direkte und indirekte Serotonin-Agonisten, wie M-CPP, Quipazin und Fenfluramin, oder Serotonin-Vorstufen, wie 5-Hydroxytryptophan (5-HTP), führen zu einem Anstieg von Kortisol und/oder ACTH, wie bei Tieren, gesunden Probanden und schizophrenen Patienten gezeigt werden konnte (Barbieri et al., 1984; Kahn et al., 1993, 1994; Lee MA et al., 1991; Owen et al., 1993).

Eine Prämedikation mit Ritanserin reduzierte einen durch M-CPP hervorgerufenen Kortisolanstieg sowohl bei gesunden Probanden (Seibyl et al., 1991) als auch bei schizophrenen Patienten (Abi-Saab et al., 2002). Lee MA et al. (1991) berichten ebenfalls eine Inhibition der durch 5-HTP induzierten Kortisolsekretion bei gesunden Probanden unter Ritanserin, während Facchinetti et al. (1987) bei Gesunden gleich bleibende Kortisolspiegel festgestellt haben. Auch eine Vorbehandlung mit Ketanserin führt zu einer Blockade der kortisolsteigernden Effekte von Fenfluramin und Quipazin, wie im Tiermodell gezeigt werden konnte (Barbieri et al., 1984).

In Analogie zu diesen Studien kann eine Prämedikation mit Clozapin und Olanzapin ebenfalls die kortisolsteigernden bzw. ACTH-steigernden Effekte von direkten oder indirekten Serotonin-Agonisten bei schizophrenen Patienten abschwächen oder gar vollständig blockieren (Kahn et al., 1993, 1994; Meltzer, 1989; Owen et al., 1993; 
Scheepers et al., 2001). Im Gegensatz hierzu hat eine Vorbehandlung mit den typischen Antipsychotika Fluphenazin und Haloperidol keinen Effekt auf eine induzierte Kortisolsekretion oder ACTH-Sekretion (Kahn et al., 1993; Owen et al., 1993).

Die klassischen Antipsychotika Fluphenazin und Haloperidol kennzeichnen sich vor allem durch einen $\mathrm{D}_{2}$-Antagonismus, während Clozapin und Olanzapin eine weitaus höhere Affinität zum 5- $\mathrm{HT}_{2 \mathrm{~A}} / 5-\mathrm{HT}_{2 \mathrm{C}}$-Rezeptor als zum $\mathrm{D}_{2}$-Rezeptor besitzen. Unter der Annahme, dass der $\mathrm{D}_{2}$-Antagonismus kaum Einfluss auf die Kortisolsekretion ausübt, erscheint es nicht verwunderlich, dass die klassischen Antipsychotika bei gesunden Probanden zumeist keine kortisolsenkenden Effekte aufweisen. Aber auch das atypische Antipsychotikum Amisulprid besitzt wie die klassischen Antipsychotika kaum Affinität zu 5-HT-Rezeptoren, sondern zeichnet sich insbesondere durch seinen Antagonismus an $\mathrm{D}_{2^{-}}$und $\mathrm{D}_{3^{-}}$-Rezeptoren aus (Schoemaker et al., 1997). Es ist daher nicht erstaunlich, dass sich für Amisulprid kein kortisolsenkender Effekt gezeigt hat (Wetzel et al., 1994). Im Gegensatz hierzu besitzen die anderen atypischen Antipsychotika Clozapin und Olanzapin und darüber hinaus auch Quetiapin und Risperidon (Cohrs et al., 2004a, 2006; De Borja Gonçalves Guerra et al., 2005; Ryan et al., 2004; Zhang et al., 2005) kortisolsenkende Eigenschaften, wobei die genannten Medikamente alle einen deutlichen $5-\mathrm{HT}_{2}$-Antagonismus aufweisen.

Im Tiermodell hat man spezifizieren können, dass diese Wirkung hauptsächlich auf dem 5- $\mathrm{HT}_{2 \mathrm{~A}}$-Antagonismus beruht. Es konnte bei Ratten gezeigt werden, dass ein mittels direkter 5- $\mathrm{HT}_{2}$-Agonisten hervorgerufener Anstieg des ACTH- bzw. Kortikosteron-Spiegels durch einen selektiven $5-\mathrm{HT}_{2 \mathrm{~A}}$-Antagonisten abgeschwächt, aber nur marginal oder gar nicht durch einen selektiven $5-\mathrm{HT}_{2 \mathrm{C}}$-Antagonisten reduziert werden kann (Hemrick-Luecke und Evans, 2002; Van de Kar et al., 2001).

Der 5-HT $\mathrm{H}_{1 \mathrm{~A}}$-Agonismus und die Komponente, welche die Wiederaufnahme von Serotonin und Noradrenalin inhibiert, dürften eher eine gesteigerte Aktivität der HPAAchse bewirken (Contesse et al., 2000; Laakmann et al., 1990).

Die Blockade von $\alpha_{1}$-Adrenozeptoren reduziert eine zuvor experimentell hervorgerufene ACTH- und Kortisolsekretion (Laakmann et al., 1986). Obwohl Ziprasidon nur sehr geringe Affinität zum $\alpha_{1}$-Adrenozeptor besitzt (Stahl und Shayegan, 2003), mag dieser auch für die durch Ziprasidon hervorgerufene Reduktion der Kortisolexkretion mitverantwortlich sein. Auch der $\mathrm{H}_{1}$-Histamin-Rezeptor-Antagonismus von Ziprasidon, der zwar noch geringer als der $\alpha_{1}$-Antagonismus ausgeprägt ist (Stahl und Shayegan, 2003), könnte zur Senkung des Kortisolspiegels beigetragen haben. So kann man im Tierversuch nachweisen, dass eine durch Histamin-Agonisten hervorgerufene Kortisolsekretion durch $\mathrm{H}_{1}$-Antagonisten inhibiert wird (Yoshida et al., 1997). Noch geringer ist der Antagonismus von Ziprasidon am muskarinergen Acetylcholin-Rezeptor vom Typ $\mathrm{M}_{1}$. Eine Senkung des Kortisolspiegels über diesen Mechanismus ist prinzipiell auch denkbar. Rao U et al. (2004) können beispielsweise die kortisolsenkende 
Wirkung Scopolamins, eines muskarinergen Acetylcholin-Rezeptor-Antagonisten, bei Frauen während einer schweren Depression als auch in der Remissionsphase zeigen.

Vor dem Hintergrund dieser Daten scheint, neben der möglichen Beteiligung des $\alpha_{1}$-Adrenozeptors, des $\mathrm{H}_{1}$-Histamin-Rezeptors und des muskarinergen AcetylcholinRezeptors vom Typ $\mathrm{M}_{1}$, die 5-HT2-antagonistische Wirkung Ziprasidons im Wesentlichen für die Verringerung der Kortisolexkretion verantwortlich zu sein.

\subsection{Ausblick}

In dieser Studie konnte erstmalig gezeigt werden, dass Ziprasidon die Schlafkontinuität verbessert, das Stadium 2 und den Tiefschlaf erhöht sowie den REM-Schlaf supprimiert.

Bei schizophrenen Patienten besteht eine Assoziation des Tiefschlaf-Defizits und der verkürzten REM-Latenz sowohl mit schlechterem Krankheitsverlauf als auch mit dem Ausmaß der Negativsymptomatik (Keshavan et al., 1995a,b; Tandon et al., 1992, 2000; Taylor et al., 1991). Durch diese Studie liegen jetzt Daten vor, die zeigen, dass Ziprasidon die gestörten Schlafparameter normalisieren kann, was somit möglicherweise auch den Verlauf und die Symptomatik einer Schizophrenie in positiver Weise beeinflusst.

Insbesondere die markante REM-Unterdrückung unterscheidet Ziprasidon von den anderen bisher untersuchten atypischen Antipsychotika und weist auf seine antidepressive Wirksamkeit hin, die auch klinisch dokumentiert wurde (Barbee et al., 2004; Nemeroff, 2005; Papakostas et al., 2004).

Nach Harvey et al. (2004a) ist Ziprasidon sogar den anderen Atypika, wie Olanzapin und Risperidon, in der Verbesserung der kognitiven Leistungsfähigkeit bei Schizophrenen überlegen. Vieles weist auf eine Beteiligung sowohl des Tiefschlafs als auch des REM-Schlafs an deklarativen Gedächtnisfunktionen (Walker MP und Stickgold, 2006) und des Schlafstadiums 2 an nicht-deklarativen Gedächtnisfunktionen hin (Smith und MacNeill, 1994; Walker MP et al., 2002). Möglicherweise ist die verbesserte kognitive Leistungsfähigkeit unter Ziprasidon neben der Wirkung auf die HPA-Achse (siehe unten) somit auch durch die Wirkung auf das Schlafstadium 2, den Tiefschlaf und den REM-Schlaf zu erklären.

Ferner belegt diese Arbeit, dass Ziprasidon wie die meisten atypischen Antipsychotika zur Abschwächung der Aktivität der HPA-Achse führt. Bei Schizophrenen besteht ein Zusammenhang zwischen einer erhöhten Aktivität der HPA-Achse und der Ausprägung von Negativsymptomen, depressiven Symptomen, kognitiven Defiziten und 
internistischen Begleiterkrankungen, die durch eine Therapie mit atypischen Antipsychotika möglicherweise positiv beeinflusst werden können.

Obwohl die atypischen Antipsychotika, wie nicht zuletzt diese Arbeit belegt, einen deutlichen Effekt auf den Schlaf und die HPA-Achse ausüben, was sich möglicherweise auch positiv auf die Symptomatik und den Krankheitsverlauf der Schizophrenie auswirkt, sind sie in diesem Zusammenhang bisher wenig untersucht worden.

Fortführende Studien unter Einschluss größerer Patienten- und Probandenkollektive sowie einer längerfristigen Arzneimittel-Applikation sollten den genannten Zusammenhang näher untersuchen.

Auch sollten weitere Studien klären, über welche Rezeptoren die genannten Effekte beim Menschen vermittelt werden, um so spezifischer wirkende Antipsychotika entwickeln zu können. 


\section{Kapitel 6}

\section{Zusammenfassung}

Ziel der vorliegenden Arbeit war die Untersuchung des Einflusses von Ziprasidon auf den Schlaf und die Kortisolausscheidung bei gesunden Probanden unter einer ungestörten Bedingung und im Rahmen eines Insomnie-Modells. Die Probanden hielten sich hierzu zweimal für zwei aufeinander folgende Tage im Abstand von fünf Tagen im Schlaflabor auf. An den zwei Abenden nahmen sie in randomisierter Reihenfolge jeweils $40 \mathrm{mg}$ Ziprasidon oder Plazebo ein. Die Medikamentengabe erfolgte doppelblind. Um Schlafstörungen und Erhöhungen des Kortisolspiegels experimentell zu provozieren, wurden die Probanden in jeder zweiten Untersuchungsnacht akustischen Reizen ausgesetzt.

In den vier Untersuchungsnächten erfolgte die polysomnographische Ableitung des Schlafs. Zur Erfassung des subjektiven Schlafempfindens füllten die Probanden standardisierte Schlaffragebögen aus. Ferner wurde der Urin in einer Abend-, Nachtund Morgenportion fraktioniert gesammelt, um die Kortisolausscheidung zu bestimmen.

Die akustische Störung des Schlafs führte zu einer Verminderung der Schlafkontinuität, zu einer Abnahme des Tiefschlaf-Anteils und zu einem Anstieg der Kortisolexkretion. Auf diese Weise konnte ein Insomnie-Modell etabliert werden, das die Schlafstörungen und die Aktivierung der Hypothalamus-Hypophysen-NebennierenAchse (Hypothalamic-pituitary-adrenal axis, HPA-Achse), wie sie bei schizophrenen Patienten vorkommen, simuliert.

Ziprasidon beeinflusste den Schlaf sowohl unter Ruhebedingungen als auch im Rahmen des Insomnie-Modells signifikant. Am ausgeprägtesten wurde der REM-Schlaf unterdrückt. Dies spiegelte sich in einer deutlichen Verminderung des REM-Anteils und der REM-Dichte sowie einer ungefähr verdoppelten REM-Latenz wider. Darüber hinaus erhöhte sich unter Ziprasidon der Tiefschlaf-Anteil. Die Schlafkontinuität nahm zu, was sich in einem Anstieg der Gesamtschlafzeit und Schlafeffizienz sowie in 
einer Verminderung der Anzahl von Aufwachvorgängen ausdrückte. Auch die per Fragebogen ermittelte subjektive Schlafeinschätzung spiegelte die schlafkonsolidierende Wirkung wider.

Die Kortisolexkretion wurde von Ziprasidon sowohl unter Ruhebedingungen als unter akustischem Stress signifikant unterdrückt. Der Medikationseffekt war dabei noch in der 14 Stunden nach Einnahme des Präparats gewonnenen morgendlichen Urinportion evident.

Anhand dieser Studie liegen jetzt erstmalig Daten vor, die darauf hinweisen, dass Ziprasidon die bei der Schizophrenie gestörten Schlafparameter normalisieren kann. Ferner belegt diese Arbeit, dass Ziprasidon einen senkenden Einfluss auf die Aktivität der HPA-Achse ausübt. Da ein Zusammenhang zwischen dem gestörtem Schlaf, einer erhöhten Aktivität der HPA-Achse und der Krankheitssymptomatik schizophrener Patienten besteht, wird die Symptomatik möglicherweise durch Ziprasidon in positiver Weise beeinflusst. Fortführende Studien sollten diesen Zusammenhang an größeren Fallzahlen und unter Einschluss schizophrener Patienten untersuchen. 
Ein Teil der Ergebnisse der vorliegenden Arbeit fand Eingang in folgende Publikationen:

Cohrs S, Meier A, Neumann AC, Jordan W, Rüther E, Rodenbeck A (2005): Improved sleep continuity and increased slow wave sleep and REM latency during ziprasidone treatment: A randomized, controlled, crossover trial of 12 healthy male subjects. J Clin Psychiatry $\underline{66}(8), 989-996$ und

Meier A, Neumann AC, Jordan W, Huether G, Rodenbeck A, Rüther E, Cohrs S (2005): Ziprasidone decreases cortisol excretion in healthy subjects.

Br J Clin Pharmacol 60(3), 330-336 
Anhang 
Tabelle A.1: Gesamtübersicht der gemessenen Schlafparameter, Teil 1 $\mathrm{Pl}=$ Plazebo, $\mathrm{Zi}=$ Ziprasidon

\begin{tabular}{|c|c|c|c|c|c|c|c|c|c|c|c|c|c|c|c|c|c|c|c|c|}
\hline \multirow[b]{3}{*}{ Proband } & \multicolumn{4}{|c|}{ SPT (min) } & \multicolumn{4}{|c|}{ TST (min) } & \multicolumn{4}{|c|}{ SE (TST/TBT)\% } & \multicolumn{4}{|c|}{ SL-1 (min) } & \multicolumn{4}{|c|}{ SL-2 (min) } \\
\hline & \multicolumn{2}{|c|}{$\begin{array}{l}\text { Nacht } 1 \\
\text { Ruhe }\end{array}$} & \multicolumn{2}{|c|}{$\begin{array}{c}\text { Nacht } 2 \\
\text { Stress }\end{array}$} & \multicolumn{2}{|c|}{$\begin{array}{l}\text { Nacht } 1 \\
\text { Ruhe }\end{array}$} & \multicolumn{2}{|c|}{$\begin{array}{c}\text { Nacht } 2 \\
\text { Stress }\end{array}$} & \multicolumn{2}{|c|}{$\begin{array}{c}\text { Nacht } 1 \\
\text { Ruhe }\end{array}$} & \multicolumn{2}{|c|}{$\begin{array}{c}\text { Nacht } 2 \\
\text { Stress }\end{array}$} & \multicolumn{2}{|c|}{$\begin{array}{c}\text { Nacht } 1 \\
\text { Ruhe }\end{array}$} & \multicolumn{2}{|c|}{$\begin{array}{c}\text { Nacht } 2 \\
\text { Stress }\end{array}$} & \multicolumn{2}{|c|}{$\begin{array}{l}\text { Nacht } 1 \\
\text { Ruhe }\end{array}$} & \multicolumn{2}{|c|}{$\begin{array}{c}\text { Nacht } 2 \\
\text { Stress }\end{array}$} \\
\hline & $\mathrm{Pl}$ & $\mathrm{Zi}$ & $\mathrm{Pl}$ & $\mathrm{Zi}$ & $\mathrm{Pl}$ & $\mathrm{Zi}$ & $\mathrm{Pl}$ & $\mathrm{Zi}$ & $\mathrm{Pl}$ & $\mathrm{Zi}$ & $\mathrm{Pl}$ & $\mathrm{Zi}$ & $\mathrm{Pl}$ & $\mathrm{Zi}$ & $\mathrm{Pl}$ & $\mathrm{Zi}$ & $\mathrm{Pl}$ & $\mathrm{Zi}$ & $\mathrm{Pl}$ & $\mathrm{Zi}$ \\
\hline 1 & 468,5 & 458,0 & 456,5 & 450,5 & 430,5 & 443,0 & 348,0 & 393,0 & 89,6 & 92,4 & 72,7 & 81,9 & 7,5 & 15,0 & 7,5 & 21,0 & 12,0 & 21,5 & 16,5 & 29,5 \\
\hline 2 & 472,5 & 472,0 & 462,5 & 476,5 & 435,5 & 458,5 & 427,5 & 461,5 & 90,8 & 95,2 & 89,1 & 96,0 & 2,0 & 7,5 & 10,5 & 0,5 & 4,0 & 9,5 & 12,0 & 4,0 \\
\hline 3 & 472,0 & 478,0 & 468,0 & 473,0 & 458,5 & 474,0 & 444,5 & 453,5 & 95,5 & 98,7 & 92,5 & 94,5 & 5,0 & 1,0 & 9,0 & 1,5 & 8,0 & 2,0 & 12,5 & 7,0 \\
\hline 4 & 469,5 & 439,5 & 470,5 & 439,5 & 453,5 & 434,0 & 395,0 & 418,5 & 94,5 & 90,3 & 82,2 & 87,2 & 6,0 & 18,0 & 7,5 & 36,5 & 10,5 & 41,0 & 10,0 & 40,5 \\
\hline 5 & 469,5 & 446,5 & 456,0 & 442,5 & 448,0 & 432,5 & 417,0 & 395,0 & 93,2 & 89,9 & 86,9 & 82,1 & 10,0 & 33,0 & 23,0 & 35,5 & 10,5 & 34,5 & 24,0 & 38,5 \\
\hline 6 & 474,5 & 474,0 & 476,5 & 470,5 & 461,0 & 463,5 & 445,5 & 451,0 & 96,6 & 96,7 & 92,9 & 93,9 & 1,0 & 3,0 & 1,5 & 7,0 & 2,5 & 5,5 & 3,0 & 10,0 \\
\hline 7 & 473,0 & 469,0 & 473,5 & 471,5 & 438,0 & 449,0 & 409,0 & 439,0 & 91,3 & 93,6 & 85,2 & 91,5 & 3,0 & 8,5 & 5,5 & 8,0 & 6,5 & 10,5 & 6,5 & 8,5 \\
\hline 8 & 461,0 & 406,0 & 324,5 & 452,0 & 442,0 & 400,5 & 278,0 & 443,5 & 92,0 & 83,4 & 57,9 & 92,4 & 20,5 & 54,5 & 10,5 & 24,0 & 19,5 & 58,0 & 155,5 & 28,0 \\
\hline 9 & 403,5 & 433,5 & 406,5 & 442,0 & 346,5 & 422,0 & 384,5 & 429,5 & 72,2 & 87,8 & 79,9 & 89,5 & 49,5 & 34,5 & 74,0 & 22,5 & 53,0 & 47,0 & 74,5 & 38,0 \\
\hline 10 & 466,0 & 475,5 & 471,0 & 434,0 & 436,0 & 463,5 & 405,0 & 417,0 & 90,8 & 96,6 & 84,4 & 86,9 & 7,5 & 2,5 & 7,0 & 40,5 & 14,0 & 4,5 & 18,5 & 46,0 \\
\hline 11 & 478,0 & 452,0 & 475,0 & 445,0 & 458,5 & 423,5 & 401,5 & 404,0 & 95,4 & 88,2 & 83,6 & 84,4 & 1,5 & 25,5 & 3,5 & 32,5 & 2,5 & 27,5 & 5,5 & 35,0 \\
\hline 12 & 420,0 & 418,5 & 422,0 & 472,0 & 396,5 & 406,5 & 355,5 & 447,0 & 82,6 & 84,7 & 74,1 & 93,0 & 54,5 & 59,0 & 55,5 & 4,0 & 60,0 & 61,5 & 58,0 & 8,5 \\
\hline MW & 460,7 & 451,9 & 446,9 & 455,8 & 433,7 & 439,2 & 392,6 & 429,4 & 90,4 & 91,5 & 81,8 & 89,4 & 14,0 & 21,8 & 17,9 & 19,5 & 16,9 & 26,9 & 33,0 & 24,5 \\
\hline $\mathrm{SD}$ & 23,5 & 23,7 & 44,3 & 15,7 & 32,6 & 23,5 & 47,1 & 23,6 & 6,8 & 4,9 & 9,8 & 4,9 & 18,5 & 19,9 & 22,8 & 14,8 & 19,2 & 21,3 & 44,4 & 15,6 \\
\hline
\end{tabular}


Tabelle A.2: Gesamtübersicht der gemessenen Schlafparameter, Teil 2 $\mathrm{Pl}=$ Plazebo, $\mathrm{Zi}=$ Ziprasidon

\begin{tabular}{|c|c|c|c|c|c|c|c|c|c|c|c|c|c|c|c|c|c|c|c|c|}
\hline & \multicolumn{4}{|c|}{ SWS-L } & \multicolumn{4}{|c|}{ REM-L (min) } & \multicolumn{4}{|c|}{$\mathrm{W}(\% \mathrm{SPT})$} & \multicolumn{4}{|c|}{ St. 1 (\% SPT) } & \multicolumn{4}{|c|}{ St. 2 (\% SPT) } \\
\hline & \multicolumn{2}{|c|}{$\begin{array}{l}\text { Nacht } 1 \\
\text { Ruhe }\end{array}$} & \multicolumn{2}{|c|}{$\begin{array}{c}\text { Nacht } 2 \\
\text { Stress }\end{array}$} & \multicolumn{2}{|c|}{$\begin{array}{l}\text { Nacht } 1 \\
\text { Ruhe }\end{array}$} & \multicolumn{2}{|c|}{$\begin{array}{c}\text { Nacht } 2 \\
\text { Stress }\end{array}$} & \multicolumn{2}{|c|}{$\begin{array}{l}\text { Nacht } 1 \\
\text { Ruhe }\end{array}$} & \multicolumn{2}{|c|}{$\begin{array}{c}\text { Nacht } 2 \\
\text { Stress }\end{array}$} & \multicolumn{2}{|c|}{$\begin{array}{c}\text { Nacht } 1 \\
\text { Ruhe }\end{array}$} & \multicolumn{2}{|c|}{$\begin{array}{c}\text { Nacht } 2 \\
\text { Stress }\end{array}$} & \multicolumn{2}{|c|}{$\begin{array}{l}\text { Nacht } 1 \\
\text { Ruhe }\end{array}$} & \multicolumn{2}{|c|}{$\begin{array}{c}\text { Nacht } 2 \\
\text { Stress }\end{array}$} \\
\hline & $\mathrm{Pl}$ & $\mathrm{Zi}$ & $\mathrm{Pl}$ & $\mathrm{Zi}$ & $\mathrm{Pl}$ & $\mathrm{Zi}$ & $\mathrm{Pl}$ & $\mathrm{Zi}$ & $\mathrm{Pl}$ & $\mathrm{Zi}$ & $\mathrm{Pl}$ & $\mathrm{Zi}$ & $\mathrm{Pl}$ & $\mathrm{Zi}$ & $\mathrm{Pl}$ & $\mathrm{Zi}$ & $\mathrm{Pl}$ & $\mathrm{Zi}$ & $\mathrm{Pl}$ & $\mathrm{Zi}$ \\
\hline 2 & 11,5 & 11,0 & 17,0 & 6,0 & 93,5 & 128,0 & 80,5 & 252,5 & 7,8 & 2,9 & 7,6 & 3,2 & 8,4 & 5,9 & 6,0 & 5,5 & 48,8 & 52,1 & 48,2 & 57,3 \\
\hline 3 & 16,5 & 10,5 & 15,0 & 9,0 & 68,5 & 272,0 & 49,0 & 147,0 & 2,9 & 0,8 & 5,0 & 4,1 & 4,7 & 7,7 & 8,0 & 6,2 & 50,6 & 61,2 & 45,7 & 47,8 \\
\hline 4 & 12,5 & 9,0 & 24,0 & 14,5 & 57,5 & 174,0 & 72,0 & 140,5 & 3,4 & 1,3 & 16,1 & 4,8 & 6,0 & 2,7 & 11,2 & 6,7 & 55,7 & 67,1 & 56,5 & 55,5 \\
\hline 5 & 9,5 & 10,5 & 15,0 & 14,5 & 137,0 & 280,5 & 91,5 & 108,8 & 4,6 & 3,1 & 8,6 & 10,7 & 3,5 & 1,6 & 5,5 & 3,5 & 52,8 & 51,0 & 61,1 & 55,9 \\
\hline 7 & 10,0 & 22,0 & 295,5 & 26,0 & 52,0 & 60,0 & 86,5 & 183,0 & 7,4 & 4,3 & 13,6 & 6,9 & 4,2 & 6,5 & 8,3 & 6,5 & 54,9 & 68,7 & 63,6 & 64,7 \\
\hline 8 & 13,0 & 10,5 & 52,5 & 11,5 & 70,0 & 78,5 & 8,0 & 147,5 & 4,1 & 1,4 & 14,3 & 1,9 & 4,7 & 2,3 & 8,0 & 2,2 & 47,8 & 52,8 & 57,6 & 57,7 \\
\hline 9 & 8,0 & 9,5 & 10,5 & 9,5 & 49,5 & 185,0 & 77,0 & 149,5 & 14,1 & 2,7 & 5,4 & 2,8 & 6,6 & 2,5 & 5,7 & 5,9 & 48,3 & 55,8 & 53,9 & 53,6 \\
\hline 10 & 25,5 & 12,0 & 22,5 & 11,0 & 99,0 & 184,5 & 0,0 & 105,0 & 6,4 & 2,5 & 14,0 & 3,9 & 9,2 & 4,1 & 6,5 & 3,2 & 46,4 & 57,5 & 45,5 & 54,3 \\
\hline 11 & 19,5 & 22,0 & 44,0 & 55,0 & 57,0 & 189,0 & 58,0 & 64,0 & 4,1 & 6,3 & 15,5 & 9,2 & 2,7 & 2,1 & 7,2 & 3,1 & 60,0 & 61,7 & 58,0 & 69,6 \\
\hline 12 & 15,0 & 18,5 & 77,0 & 18,0 & 82,0 & 64,0 & 110,5 & 248,0 & 5,6 & 2,9 & 15,8 & 5,3 & 16,4 & 3,2 & 6,5 & 6,7 & 43,9 & 62,0 & 45,4 & 61,7 \\
\hline MW & 14,0 & 13,9 & 51,3 & 23,3 & 75,2 & 152,1 & 76,0 & 159,7 & 6,0 & 2,8 & 12,2 & 5,8 & 7,0 & 4,6 & 7,9 & 5,6 & 50,9 & 59,1 & 52,4 & 58,0 \\
\hline
\end{tabular}


Tabelle A.3: Gesamtübersicht der gemessenen Schlafparameter, Teil 3

$\mathrm{Pl}=$ Plazebo, $\mathrm{Zi}=$ Ziprasidon

\begin{tabular}{|c|c|c|c|c|c|c|c|c|c|c|c|c|c|c|c|c|c|c|c|c|c|c|c|c|}
\hline & \multicolumn{4}{|c|}{ SWS (\% SPT) } & \multicolumn{4}{|c|}{ REM (\% SPT) } & \multicolumn{4}{|c|}{ Anz. Aufwachv. } & \multicolumn{4}{|c|}{ REM-Dichte } & \multicolumn{4}{|c|}{ REM-Dichte-1 } & \multicolumn{4}{|c|}{ PLMS } \\
\hline & \multicolumn{2}{|c|}{$\begin{array}{c}\text { Nacht } 1 \\
\text { Ruhe }\end{array}$} & \multicolumn{2}{|c|}{$\begin{array}{c}\text { Nacht } 2 \\
\text { Stress }\end{array}$} & \multicolumn{2}{|c|}{$\begin{array}{c}\text { Nacht } 1 \\
\text { Ruhe }\end{array}$} & \multicolumn{2}{|c|}{$\begin{array}{c}\text { Nacht } 2 \\
\text { Stress } \\
\end{array}$} & \multicolumn{2}{|c|}{$\begin{array}{l}\text { Nacht } 1 \\
\text { Ruhe }\end{array}$} & \multicolumn{2}{|c|}{$\begin{array}{c}\text { Nacht } 2 \\
\text { Stress } \\
\end{array}$} & \multicolumn{2}{|c|}{$\begin{array}{c}\text { Nacht } 1 \\
\text { Ruhe }\end{array}$} & \multicolumn{2}{|c|}{$\begin{array}{c}\text { Nacht } 2 \\
\text { Stress } \\
\end{array}$} & \multicolumn{2}{|c|}{$\begin{array}{c}\text { Nacht } 1 \\
\text { Ruhe }\end{array}$} & \multicolumn{2}{|c|}{$\begin{array}{c}\text { Nacht } 2 \\
\text { Stress }\end{array}$} & \multicolumn{2}{|c|}{$\begin{array}{c}\text { Nacht } 1 \\
\text { Ruhe }\end{array}$} & \multicolumn{2}{|c|}{$\begin{array}{c}\text { Nacht } 2 \\
\text { Stress } \\
\end{array}$} \\
\hline & $\mathrm{Pl}$ & $\mathrm{Zi}$ & $\mathrm{Pl}$ & $\mathrm{Zi}$ & $\mathrm{Pl}$ & $\mathrm{Zi}$ & $\mathrm{Pl}$ & $\mathrm{Zi}$ & $\mathrm{Pl}$ & $\mathrm{Zi}$ & $\mathrm{Pl}$ & $\mathrm{Zi}$ & $\mathrm{Pl}$ & $\mathrm{Zi}$ & $\mathrm{Pl}$ & $\mathrm{Zi}$ & $\mathrm{Pl}$ & $\mathrm{Zi}$ & $\mathrm{Pl}$ & $\mathrm{Zi}$ & $\mathrm{Pl}$ & $\mathrm{Zi}$ & $\mathrm{Pl}$ & $\mathrm{Zi}$ \\
\hline 1 & 17,5 & 16,9 & 11,3 & 9,8 & 13,1 & 3,0 & 13,0 & 6,4 & 48,0 & 20,0 & 55,0 & 25,0 & 1,0 & 0,2 & 1,0 & 0,6 & 0,7 & 1,0 & 1,4 & 0,7 & 0,0 & 0,0 & 0,0 & 0,0 \\
\hline 2 & 12,1 & 27,1 & 11,7 & 25,7 & 23,0 & 12,0 & 26,6 & 8,4 & 47,0 & 22,0 & 36,0 & 21,0 & 1,1 & 0,4 & 1,3 & 0,1 & 1,2 & 0,7 & 1,5 & 0,1 & 0,0 & 201,0 & 21,0 & 85,0 \\
\hline 3 & 18,8 & 22,4 & 16,0 & 24,7 & 23,1 & 7,9 & 25,2 & 17,1 & 22,0 & 7,0 & 37,0 & 24,0 & 1,4 & 0,9 & 1,2 & 1,6 & 0,6 & 0,5 & 0,9 & 0,7 & 0,0 & 173,0 & 0,0 & 12,0 \\
\hline I & 15,5 & 21,9 & 3,3 & 19,9 & 19,5 & 7,1 & 12,9 & 13,1 & 25,0 & 13,0 & 35,0 & 26,0 & 2,7 & 0,6 & 3,1 & 0,4 & 2,5 & 1,1 & 1,8 & 0,4 & 5,0 & 0,0 & 144,0 & 5,0 \\
\hline 5 & 21,6 & 39,4 & 7,8 & 19,2 & 17,5 & 4,9 & 17,1 & 10,6 & 21,0 & 14,0 & 45,0 & 30,0 & 0,4 & 0,2 & 0,9 & 0,7 & 0,1 & 0,4 & 0,4 & 1,3 & 0,0 & 38,0 & 0,0 & 0,0 \\
\hline 6 & 17,3 & 22,6 & 12,0 & 20,2 & 22,6 & 16,4 & 18,1 & 11,2 & 19,0 & 18,0 & 47,0 & 28,0 & 1,2 & 0,6 & 1,8 & 0,8 & 0,9 & 1,3 & 0,5 & 0,6 & 0,0 & 4,0 & 23,0 & 10,0 \\
\hline 7 & 10,3 & 8,7 & 1,0 & 6,5 & 23,3 & 11,8 & 13,5 & 15,5 & 28,0 & 27,0 & 44,0 & 40,0 & 2,1 & 1,7 & 2,1 & 1,4 & 2,1 & 1,5 & 1,6 & 0,7 & 0,0 & 0,0 & 0,0 & 0,0 \\
\hline 8 & 22,9 & 36,0 & 5,6 & 24,6 & 20,5 & 7,5 & 14,5 & 13,6 & 15,0 & 11,0 & 29,0 & 15,0 & 1,3 & 0,6 & 2,4 & 1,4 & 1,9 & 2,0 & 2,3 & 1,0 & 0,0 & 0,0 & 38,0 & 4,0 \\
\hline 9 & 14,5 & 21,8 & 14,4 & 18,4 & 16,5 & 17,2 & 20,7 & 19,2 & 32,0 & 20,0 & 36,0 & 18,0 & 2,6 & 0,9 & 2,1 & 1,0 & 1,0 & 0,3 & 2,6 & 1,0 & 4,0 & 0,0 & 0,0 & 0,0 \\
\hline 10 & 14,2 & 25,8 & 9,1 & 21,0 & 23,8 & 10,1 & 24,8 & 17,6 & 29,0 & 18,0 & 49,0 & 26,0 & 1,3 & 0,3 & 1,1 & 1,3 & 0,8 & 0,1 & 0,3 & 2,0 & 0,0 & 0,0 & 0,0 & 0,0 \\
\hline 11 & 8,0 & 11,4 & 4,3 & 7,5 & 25,2 & 18,5 & 15,1 & 10,6 & 34,0 & 11,0 & 45,0 & 19,0 & 1,2 & 0,6 & 2,1 & 1,0 & 0,7 & 0,8 & 2,3 & 0,6 & 37,0 & 65,0 & 46,0 & 52,0 \\
\hline 12 & 19,6 & 13,9 & 4,4 & 9,9 & 14,4 & 18,0 & 27,8 & 16,5 & 29,0 & 16,0 & 34,0 & 27,0 & 1,1 & 0,8 & 0,7 & 0,5 & 0,8 & 0,6 & 1,0 & 0,6 & 8,0 & 0,0 & 82,0 & 19,0 \\
\hline MW & 16,0 & 22,3 & 8,4 & 17,3 & 20,2 & 11,2 & 19,1 & 13,3 & 29,1 & 16,4 & 41,0 & 24,9 & 1,4 & 0,6 & 1,7 & 0,9 & 1,1 & 0,9 & 1,4 & 0,8 & 4,5 & 40,1 & 29,5 & 15,6 \\
\hline SD & 4,5 & 9,1 & 4,8 & 7,0 & 4,0 & 5,3 & 5,7 & 4,0 & 10,2 & 5,6 & 7,6 & 6,5 & 0,7 & 0,4 & 0,7 & 0,5 & 0,7 & 0,5 & 0,8 & 0,5 & 10,6 & 71,8 & 44,3 & 26,4 \\
\hline
\end{tabular}


Tabelle A.4: Mittelwerte der REM-Parameter über Nacht 1 und Nacht 2

\begin{tabular}{|c|c|c|c|c|c|c|}
\hline & \multicolumn{2}{|c|}{ REM (\% SPT) } & \multicolumn{2}{|c|}{ REM-L (min) } & \multicolumn{2}{|c|}{ REM-Dichte } \\
\hline & Nacht $1+2$ & Nacht $1+2$ & Nacht $1+2$ & Nacht $1+2$ & Nacht $1+2$ & Nacht $1+2$ \\
\hline Proband & Plazebo & Ziprasidon & Plazebo & Ziprasidon & Plazebo & Ziprasidon \\
\hline 1 & 13,1 & 4,7 & 159,8 & 154,8 & 1,0 & 0,4 \\
\hline 2 & 24,8 & 10,2 & 87,0 & 190,3 & 1,2 & 0,2 \\
\hline 3 & 24,2 & 12,5 & 58,8 & 209,5 & 1,3 & 1,2 \\
\hline 4 & 16,2 & 10,1 & 64,8 & 157,3 & 2,9 & 0,5 \\
\hline 5 & 17,3 & 7,8 & 114,3 & 194,7 & 0,6 & 0,5 \\
\hline 6 & 20,3 & 13,8 & 48,0 & 135,5 & 1,5 & 0,7 \\
\hline 7 & 18,4 & 13,7 & 69,3 & 121,5 & 2,1 & 1,5 \\
\hline 8 & 17,5 & 10,6 & 39,0 & 113,0 & 1,8 & 1,0 \\
\hline 9 & 18,6 & 18,2 & 63,3 & 167,3 & 2,3 & 0,9 \\
\hline 10 & 24,3 & 13,9 & 49,5 & 144,8 & 1,2 & 0,8 \\
\hline 11 & 20,1 & 14,5 & 57,5 & 126,5 & 1,6 & 0,8 \\
\hline 12 & 21,1 & 17,3 & 96,3 & 156,0 & 0,9 & 0,6 \\
\hline MW & 19,7 & 12,3 & 75,6 & 155,9 & 1,5 & 0,8 \\
\hline SD & 3,6 & 3,8 & 34,2 & 30,3 & 0,7 & 0,4 \\
\hline
\end{tabular}


Tabelle A.5: Gesamtübersicht der pro Sammelzeitraum im Urin gemessenen Kortisolexkretion in $\mu \mathrm{g}$

\begin{tabular}{|c|c|c|c|c|c|c|c|c|c|c|c|c|}
\hline & & & cht 1 , Ruh & bedingung & & & & & cht 2 , akus & tischer Str & & \\
\hline & & Plazebo & & & Ziprasidon & & & Plazebo & & & Ziprasidon & \\
\hline Proband & $19-23: 00$ & 23-07:00 & $07-11: 00$ & 19-23:00 & $23-07: 00$ & 07-11:00 & $19-23: 00$ & $23-07: 00$ & 07-11:00 & 19-23:00 & $23-07: 00$ & 07-11:00 \\
\hline 1 & 8,5 & 22,2 & 52,6 & 5,2 & 13,6 & 39,6 & 9,4 & 23,3 & 8,8 & 3,6 & 11,0 & 23,8 \\
\hline 2 & 6,1 & 27,6 & 20,6 & 7,8 & 24,1 & 18,8 & 4,9 & 24,1 & 18,6 & 7,8 & 21,0 & 24,0 \\
\hline 3 & 7,3 & 9,4 & 15,5 & 7,6 & 6,4 & 6,6 & 6,4 & 20,6 & 18,1 & 6,2 & 10,5 & 8,0 \\
\hline 4 & 14,5 & 11,6 & 17,8 & 7,0 & 6,9 & 30,7 & 3,4 & 16,5 & 37,1 & 7,5 & 15,3 & 23,3 \\
\hline 5 & 3,9 & 13,6 & 8,3 & 6,0 & 9,7 & 18,6 & 4,2 & 23,1 & 27,3 & 5,6 & 12,0 & 18,3 \\
\hline 6 & 5,2 & 17,8 & 25,3 & 10,9 & 11,5 & 18,1 & 11,4 & 32,5 & 52,7 & 8,1 & 11,0 & 20,8 \\
\hline 7 & 4,3 & 15,1 & 36,8 & 3,7 & 6,5 & 17,4 & 6,8 & 20,3 & 28,9 & 2,4 & 4,4 & 12,5 \\
\hline 8 & 5,5 & 11,6 & 23,8 & 7,3 & 7,1 & 15,0 & 8,9 & 16,0 & 44,2 & 6,5 & 7,6 & 16,5 \\
\hline 9 & 2,1 & 9,3 & 11,7 & 8,3 & 3,3 & 8,4 & 9,8 & 12,4 & 16,1 & 5,5 & 7,7 & 11,0 \\
\hline 10 & 3,2 & 15,9 & 45,0 & 15,3 & 11,7 & 14,6 & 7,2 & 46,3 & 42,4 & 13,8 & 21,6 & 18,8 \\
\hline 11 & 5,3 & 3,9 & 3,8 & 6,3 & 3,5 & 9,5 & 5,7 & 13,3 & 34,6 & 7,9 & 7,8 & 18,9 \\
\hline MW & 6,0 & 14,3 & 23,8 & 7,8 & 9,5 & 17,9 & 7,1 & 22,6 & 29,9 & 6,8 & 11,8 & 17,8 \\
\hline SD & 3,4 & 6,5 & 15,3 & 3,1 & 5,9 & 9,7 & 2,5 & 9,7 & 13,7 & 3,0 & 5,5 & 5,4 \\
\hline
\end{tabular}


Tabelle A.6: Mittelwerte der Kortisolexkretion in $\mu$ g über Nacht 1 und Nacht 2

\begin{tabular}{|c|c|c|c|c|c|c|}
\hline & Nacht $1+2$ & Nacht $1+2$ & Nacht $1+2$ & Nacht $1+2$ & Nacht $1+2$ & Nacht $1+2$ \\
\hline & Plazebo & Ziprasidon & Plazebo & Ziprasidon & Plazebo & Ziprasidon \\
\hline Proband & $19-23: 00$ & 19-23:00 & $23-07: 00$ & 23-07:00 & $07-11: 00$ & 07-11:00 \\
\hline 1 & 8,9 & 4,4 & 22,7 & 12,3 & 30,7 & 31,7 \\
\hline 2 & 5,5 & 7,8 & 25,8 & 22,6 & 19,6 & 21,4 \\
\hline 3 & 6,8 & 6,9 & 15,0 & 8,4 & 16,8 & 7,3 \\
\hline 4 & 9,0 & 7,2 & 14,0 & 11,1 & 27,5 & 27,0 \\
\hline 5 & 4,1 & 5,8 & 18,4 & 10,8 & 17,8 & 18,5 \\
\hline 6 & 8,3 & 9,5 & 25,2 & 11,3 & 39,0 & 19,4 \\
\hline 7 & 5,5 & 3,1 & 17,7 & 5,5 & 32,8 & 15,0 \\
\hline 8 & 7,2 & 6,9 & 13,8 & 7,4 & 34,0 & 15,8 \\
\hline 9 & 5,9 & 6,9 & 10,8 & 5,5 & 13,9 & 9,7 \\
\hline 10 & 5,2 & 14,6 & 31,1 & 16,7 & 43,7 & 16,7 \\
\hline 11 & 5,5 & 7,1 & 8,6 & 5,7 & 19,2 & 14,2 \\
\hline MW & 6,5 & 7,3 & 18,5 & 10,6 & 26,8 & 17,9 \\
\hline SD & 1,6 & 3,0 & 7,0 & 5,2 & 10,0 & 7,0 \\
\hline
\end{tabular}




\section{Literaturverzeichnis}

Abi-Dargham A (2004): Do we still believe in the dopamine hypothesis? New data bring new evidence. Int J Neuropsychopharmacol $\underline{7}, 1-5$

Abi-Saab W, Seibyl JP, et al. (2002): Ritanserin antagonism of m-chlorophenylpiperazine effects in neuroleptic-free schizophrenics patients: support for serotonin-2 receptor modulation of schizophrenia symptoms. Psychopharmacology 162, 55-62

Adam K, Oswald I (1977): Sleep is for tissue restoration. J R Coll Physicians Lond 11, 376-388

Adam K, Allen S, Carruthers-Jones I, Oswald I, Spence M (1976): Mesoridazine and human sleep. Br J Clin Pharmacol יㅗ 157-163

Addington D, Addington J (1990): Depression, dexamethasone nonsuppression and negative symptoms in schizophrenia. Can J Psychiatry 35, 430-433

Adityanjee, Schulz SC (2002): Clinical use of quetiapine in disease states other than schizophrenia. J Clin Psychiatry 63, 32-38

Altamura C, Guercetti G, Percudani M (1989): Dexamethasone suppression test in positive and negative schizophrenia. Psychiatry Res $30,69-75$

Anand A, Malison R, Mc Dougle CJ, Price LH (1995): Antiglucocorticoid treatment of refractory depression with ketoconazole: a case report. Biol Psychiatry 37, 338-340

Arana GW, Baldessarini RJ, Ornsteen M (1985): The dexamethasone suppression test for diagnosis and prognosis in psychiatry. Commentary and review. Arch Gen Psychiatry $\underline{42}$, $1193-1204$

Armitage R, Emslie G, Rintelmann J (1997): The effect of fluoxetine on sleep EEG in childhood depression: a preliminary report. Neuropsychopharmacology 17, 241-245

Aserinsky E, Kleitman N (1953): Regularly occurring periods of eye motility, and concomitant phenomena, during sleep. Science $\underline{118}$, 273-274

Aslan S, Isik E, Cosar B (2002): The effects of mirtazapine on sleep: a placebo controlled, double-blind study in young healthy volunteers. Sleep $25,677-679$

Asnis GM, Eisenberg J, Lemus CZ, Halbreich U (1986): Dexamethasone suppression test in schizophrenia. A study and review. Neuropsychobiology 15, 109-113

Bandelow B, Müller P, Frick U, Gaebel W, Linden M, Müller-Spahn F, Pietzcker A, Tegeler J (1992): Depressive syndromes in schizophrenic patients under neuroleptic therapy. ANI 
Study Group Berlin, Düsseldorf, Göttingen, Munich, Federal Republic of Germany. Eur Arch Psychiatry Clin Neurosci 241, 291-295

Baptista T, Alastre T, Contreras Q, Martinez JL, de Baptista EA, Páez X, Hernández L (1997a): Effects of the antipsychotic drug sulpiride on reproductive hormones in healthy men: relationship with body weight regulation. Pharmacopsychiatry $\underline{30}$, 250-255

Baptista T, Molina MG, Martinez JL, de Quijada M, de Cuesta IC, Acosta A, Páez X, Martinez JM, Hernández L (1997b): Effects of the antipsychotic drug sulpiride on reproductive hormones in healthy premenopausal women: relationship with body weight regulation. Pharmacopsychiatry $\underline{30}, 256-262$

Barbee JG, Conrad EJ, Jamhour NJ (2004): The effectiveness of olanzapine, risperidone, quetiapine, and ziprasidone as augmentation agents in treatment-resistant major depressive disorder. J Clin Psychiatry 65, 975-981

Barbieri C, Sala M, Bigatti G, Rauhe WG, Guffanti A, Diena A, Scorza D, Bevilacqua M, Norbiato G (1984): Serotonergic regulation of cortisol secretion in dogs. Endocrinology $\underline{115}, 748-751$

Belenky G, Wesensten NJ, Thorne DR, Thomas ML, Sing HC, Redmond DP, Russo MB, Balkin TJ (2003): Patterns of performance degradation and restoration during sleep restriction and subsequent recovery: a sleep dose-response study. J Sleep Res $\underline{12}$, 1-12

Benca RM: Mood Disorders; in: Principles and practice of sleep medicine; hrsg. v. Kryger MH, Roth T, Dement WC; W. B. Saunders Company, Philadelphia 2000, 1140-1157

Benca RM, Obermeyer WH, Thisted RA, Gillin JC (1992): Sleep and psychiatric disorders. A meta-analysis. Arch Gen Psychiatry $\underline{49}, 651-668$

Benkert O, Hippius H, Kompendium der psychiatrischen Pharmakotherapie. 4. Auflage, Springer, Berlin 2003

Benson KL, Zarcone VP (1993): Rapid eye movement sleep eye movements in schizophrenia and depression. Arch Gen Psychiatry 50, 474-482

Benson KL, Zarcone VP: Schizophrenia; in: Principles and practice of sleep medicine; hrsg. v. Kryger MH, Roth T, Dement WC; W. B. Saunders Company, Philadelphia 2000, $1159-1167$

Berger M, Riemann D, Höchli D, Spiegel R (1989): The cholinergic rapid eye movement sleep induction test with RS-86. State or trait marker of depression? Arch Gen Psychiatry $\underline{46}, 421-428$

Berger RJ, Phillips NH (1995): Energy conservation and sleep. Behav Brain Res $\underline{69}$, 65-73

Blum A, Girke W (1973): Marked increase in REM sleep produced by a new antipsychotic compound. Clin Electroencephalogr $\underline{4}, 80-84$

Bogerts B, Meertz E, Schönfeldt-Bausch R (1985): Basal ganglia and limbic system pathology in schizophrenia. A morphometric study of brain volume and shrinkage. Arch Gen Psychiatry $\underline{42}, 784-791$ 
Bonnet MH (1985): Effect of sleep disruption on sleep, performance, and mood. Sleep $\underline{8}$, $11-19$

Borbély AA (1982): A two process model of sleep regulation. Hum Neurobiol 1, 195-204

Borbély A, Das Geheimnis des Schlafs. Neue Wege und Erkenntnisse der Forschung. Universität Zürich, Zürich 1998 (http://www.pharma.unizh.ch/sleep/buch)

Borbély AA, Wirz-Justice A (1982): Sleep, sleep deprivation and depression. A hypothesis derived from a model of sleep regulation. Hum Neurobiol $\underline{1}, 205-210$

Borbély AA, Achermann P: Sleep homeostasis and models of sleep regulation; in: Principles and practice of sleep medicine; hrsg. v. Kryger MH, Roth T, Dement WC; W. B. Saunders Company, Philadelphia 2000, 377-390

Bortz J, Statistik für Human- und Sozialwissenschaftler. 6. Auflage; Springer, Heidelberg 2005

Brambilla F, Guerrini A, Guastalla A, Rovere C, Riggi F (1975): Neuroendocrine effects of haloperidol therapy in chronic schizophrenia. Psychopharmacologia $\underline{44}, 17-22$

Brannen JO, Jewett RE (1969): Effects of selected phenothiazines on REM sleep in schizophrenics. Arch Gen Psychiatry 21, 284-290

Breier A, Buchanan RW, Waltrip RW, Listwak S, Holmes C, Goldstein DS (1994): The effect of clozapine on plasma norepinephrine: relationship to clinical efficacy. Neuropsychopharmacology $\underline{10}, 1-7$

Brown ES, Varghese FP, Mc Ewen BS (2004): Association of depression with medical illness: does cortisol play a role? Biol Psychiatry $55,1-9$

Buysse DJ, Reynolds CF, Hoch CC, Houck PR, Kupfer DJ, Mazumdar S, Frank E (1996): Longitudinal effects of nortriptyline on EEG sleep and the likelihood of recurrence in elderly depressed patients. Neuropsychopharmacology $14,243-252$

Calabrese JR, Keck PE, Macfadden W, Minkwitz M, Ketter TA, Weisler RH, Cutler AJ, Mc Coy R, Wilson E, Mullen J (2005): A randomized, double-blind, placebo-controlled trial of quetiapine in the treatment of bipolar I or II depression. Am J Psychiatry 162, $1351-1360$

Caldwell DF, Domino EF (1967): Electroencephalographic and eye movement patterns during sleep in chronic schizophrenic patients. Electroencephalogr Clin Neurophysiol 22, $414-420$

Caley CF, Cooper CK (2002): Ziprasidone: the fifth atypical antipsychotic. Ann Pharmacother $36,839-851$

Carroll BJ (1982): Clinical applications of the dexamethasone suppression test for endogenous depression. Pharmacopsychiatria 15, 19-25

Cesková E, Drybcák P, Hrobar P, Lorenc M, Procházková H, Spacek J (2001): The changes of biological markers and treatment efficacy in schizophrenia. Prog Neuropsychopharmacol Biol Psychiatry 25, 323-335 
Chang PP, Ford DE, Mead LA, Cooper-Patrick L, Klag MJ (1997): Insomnia in young men and subsequent depression. The Johns Hopkins Precursors Study. Am J Epidemiol 146, $105-114$

Chrousos GP, Gold PW (1992): The concepts of stress and stress system disorders. Overview of physical and behavioral homeostasis. JAMA $267,1244-1252$

Cipolli C, Bolzani R, Tuozzi G, Fagioli I (2001): Active processing of declarative knowledge during REM-sleep dreaming. J Sleep Res 10, 277-284

Clarenbach P, Prunkl R, Riegler M, Cramer H (1978): Effects of haloperidol on afternoon sleep and on the secretion of growth hormone in man. Neuroscience $\underline{3}, 345-348$

Cluydts R, De Roeck J, Cosyns P, Lacante P (1995): Antagonizing the effects of experimentally induced sleep disturbance in healthy volunteers by lormetazepam and zolpidem. $\mathrm{J}$ Clin Psychopharmacol 15, 132-137

Cohrs S, Pohlmann K, Guan Z, Jordan W, Meier A, Huether G, Rüther E, Rodenbeck A (2004a): Quetiapine reduces nocturnal urinary cortisol excretion in healthy subjects. Psychopharmacology 174, 414-420

Cohrs S, Rodenbeck A, Guan Z, Pohlmann K, Jordan W, Meier A, Rüther E (2004b): Sleep-promoting properties of quetiapine in healthy subjects. Psychopharmacology $\underline{174}$, $421-429$

Cohrs S, Röher C, Jordan W, Meier A, Huether G, Wuttke W, Rüther E, Rodenbeck A (2006): The atypical antipsychotics olanzapine and quetiapine, but not haloperidol, reduce ACTH and cortisol secretion in healthy subjects. Psychopharmacology 185, 11-18

Contesse V, Lefebvre H, Lenglet S, Kuhn JM, Delarue C, Vaudry H (2000): Role of 5-HT in the regulation of the brain-pituitary-adrenal axis: effects of 5-HT on adrenocortical cells. Can J Physiol Pharmacol 78, 967-983

Coppen A, Abou-Saleh M, Milln P, Metcalfe M, Harwood J, Bailey J (1983): Dexamethasone suppression test in depression and other psychiatric illness. Br J Psychiatry 142, 498-504

Cross EM, Chaffin WW (1982): Use of the binomial theorem in interpreting results of multiple tests of significance. Educ Psychol Meas 42, 25-34

Cuesta MJ, Peralta V, Zarzuela A (2001): Effects of olanzapine and other antipsychotics on cognitive function in chronic schizophrenia: a longitudinal study. Schizophr Res $\underline{48}$, $17-28$

Curtis VA, Wright P, Reveley A, Kerwin R, Lucey JV (1995): Effect of clozapine on dfenfluramine-evoked neuroendocrine responses in schizophrenia and its relationship to clinical improvement. Br J Psychiatry 166, 642-646

Daniel DG (2003): Tolerability of ziprasidone: an expanding perspective. J Clin Psychiatry $\underline{64}, 40-49$

Daniel DG, Copeland LF (2000): Ziprasidone: comprehensive overview and clinical use of a novel antipsychotic. Expert Opin Investig Drugs $\underline{9}$, 819-828 
Daniel DG, Zimbroff DL, Potkin SG, Reeves KR, Harrigan EP, Lakshminarayanan M (1999): Ziprasidone $80 \mathrm{mg} /$ day and $160 \mathrm{mg} /$ day in the acute exacerbation of schizophrenia and schizoaffective disorder: a 6-week placebo-controlled trial. Neuropharmacology 20, $491-505$

Daniel DG, Potkin SG, Reeves KR, Swift RH, Harrigan EP (2001): Intramuscular (IM) ziprasidone $20 \mathrm{mg}$ is effective in reducing acute agitation associated with psychosis: a double-blind, randomized trial. Psychopharmacology 155, 128-134

Dawson D, Reid K (1997): Fatigue, alcohol and performance impairment. Nature 388, 235

De Borja Gonçalves Guerra A, Castel S, Benedito-Silva AA, Calil HM (2005): Neuroendocrine effects of quetiapine in healthy volunteers. Int J Neuropsychopharmacol $\underline{8}, 49-57$

De Gennaro L, Ferrara M, Bertini M (2000): The relationship between frequency of rapid eye movements in REM sleep and SWS rebound. J Sleep Res $\underline{9}$, 155-159

Deklaration von Helsinki: World medical association declaration of helsinki. Ethical principles for medical research involving human subjects. World medical association general assembly, Tokyo 2004 (http://www.wma.net/e/policy/b3.htm)

De Koninck J, Lorrain D, Christ G, Proulx G, Coulombe D (1989): Intensive language learning and increases in rapid eye movement sleep: evidence of a performance factor. Int J Psychophysiol $\underline{8}, 43-47$

De Koning P, De Vries MH (1995): A comparison of the neuro-endocrinological and temperature effects of DU 29894, flesinoxan, sulpiride and haloperidol in normal volunteers. Br J Clin Pharmacol 39, 7-14

Delgado PL (2000): Depression: the case for a monoamine deficiency. J Clin Psychiatry 61, $7-11$

Dement W, Kleitman N (1957): The relation of eye movements during sleep to dream activity: an objective method for the study of dreaming. J Exp Psychol 53, 339-346

Deutsche Rentenversicherung: Statistik der deutschen Rentenversicherung. Deutsche Rentenversicherung in Zahlen 2006. Deutsche Rentenversicherung Bund, Geschäftsbereich Presse- und Öffentlichkeitsarbeit, Kommunikation, Berlin 2006

Dinan TG (2004): Stress and the genesis of diabetes mellitus in schizophrenia. Br J Psychiatry $\underline{184}, 72-75$

Doering S, Wedekind D, Pilz J, Bandelow B, Adler L, Huether G (2001): Cortisol in nighturine: Introduction of a research method in psychoneuroendocrinology. Z Psychosom Med Psychother $\underline{47}, 42-57$

Eichenbaum H, Schoenbaum G, Young B, Bunsey M (1996): Functional organization of the hippocampal memory system. Proc Natl Acad Sci USA 93, 13500-13507

Facchinetti F, Martignoni E, Nappi G, Marini S, Petraglia F, Sandrini G, Genazzani AR (1987): Ritanserin, a serotonin-S2 receptor antagonist, does not prevent 5hydroxytryptophan-induced beta-EP, beta-LPH and cortisol secretion. Horm Res $\underline{27}$, $42-46$ 
Falkai P, Bogerts B (1986): Cell loss in the hippocampus of schizophrenics. Eur Arch Psychiatry Neurol Sci $236,154-161$

Feige B, Voderholzer U, Riemann D, Dittmann R, Hohagen F, Berger M (2002): Fluoxetine and sleep EEG: effects of a single dose, subchronic treatment, and discontinuation in healthy subjects. Neuropsychopharmacology $\underline{26}, 246-58$

Feinberg I, Koresko RL, Gottlieb F (1965): Further observations on electrophysiological sleep patterns in schizophrenia. Compr Psychiatry $\underline{6}$, 21-24

Feuillade P, Pringuey D, Belugou JL, Robert P, Darcourt G (1992): Trimipramine: acute and lasting effects on sleep in healthy and major depressive subjects. J Affect Disord $\underline{24}$, $135-145$

Fischer J, Mayer G, Peter JH, Riemann D, Sitter H, Nicht-erholsamer Schlaf. Leitlinie „S2“ der Deutschen Gesellschaft für Schlafforschung und Schlafmedizin (DGSM). Arbeitsgemeinschaft der Wissenschaftlichen Medizinischen Fachgesellschaften (AWMF), Düsseldorf 2001 (http://www. uni-duesseldorf.de/AWMF/1l/063-001.htm)

Fischer-Cornelssen KA: Fischer Somatische Symptome oder Unerwünschte Effekte Check List (FSUCL); in: Internationale Skalen für Psychiatrie; hrsg. v. Collegium Internationale Psychiatriae Scalarum (CIPS); Beltz Test GmbH, Weinheim 1986

Foulkes WD (1962): Dream reports from different stages of sleep. J Abnorm Soc Psychol $\underline{65}, 14-25$

Frank MG, Benington JH (2006): The role of sleep in memory consolidation and brain plasticity: dream or reality? Neuroscientist $\underline{12}, 477-488$

Freemon FR (1973): Clinical pharmacology of sleep: a critical review of all-night electroencephalographic studies. Behav Neuropsychiatry $\underline{4-5}, 49-60$

Gaillard JM, Moneme A (1977): Modification of dream content after preferential blockade of mesolimbic and mesocortical dopaminergic systems. J Psychiatr Res 13, 247-256

Gais S, Born J (2004): Declarative memory consolidation: mechanisms acting during human sleep. Learn Mem 11, 679-685

Gallagher P, Watson S, Smith MS, Ferrier IN, Young AH (2005): Effects of adjunctive mifepristone (RU-486) administration on neurocognitive function and symptoms in schizophrenia. Biol Psychiatry 57, 155-161

Ganguli R, Reynolds CF, Kupfer DJ (1987): Electroencephalographic sleep in young, nevermedicated schizophrenics. A comparison with delusional and nondelusional depressives and with healthy controls. Arch Gen Psychiatry 44, 36-44

Giertz H, Flohé L, Göthert M, Peskar BA, Resch K: Mediatoren der Entzündung und Allergie; in: Allgemeine und spezielle Pharmakologie und Toxikologie; hrsg. v. Forth W, Henschler D, Rummel W, Starke K; Spektrum, Akad. Verl., Heidelberg 1996, 319-362

Gillin JC, van Kammen DP, Post R, Bunney WE (1977): Effects of prolonged administration of pimozide on sleep-EEG patterns in psychiatric patients. Commun Psychopharmacol $\underline{1}, 225-232$ 
Gillin JC, Wyatt RJ, Fram D, Snyder F (1978): The relationship between changes in REM sleep and clinical improvement in depressed patients treated with amitriptyline. Psychopharmacology $\underline{59}, 267-272$

Gillin JC, Sutton L, Ruiz C, Kelsoe J, Dupont RM, Darko D, Risch SC, Golshan S, Janowsky D (1991): The cholinergic rapid eye movement induction test with arecoline in depression. Arch Gen Psychiatry $\underline{48}$, 264-270

Gillin JC, Jernajczyk W, Valladares-Neto DC, Golshan S, Lardon M, Stahl SM (1994): Inhibition of REM sleep by ipsapirone, a $5-\mathrm{HT}_{1 \mathrm{~A}}$ agonist, in normal volunteers. Psychopharmacology $\underline{116}, 433-436$

Gillin JC, Sohn JW, Stahl SM, Lardon M, Kelsoe J, Rapaport M, Ruiz C, Golshan S (1996): Ipsapirone, a $5-\mathrm{HT}_{1 \mathrm{~A}}$ agonist, suppresses REM sleep equally in unmedicated depressed patients and normal controls. Neuropsychopharmacology 15, 109-115

Glaeske G, Janhsen K, GEK-Arzneimittel-Report 2006. Auswertungsergebnisse der GEKArzneimitteldaten aus den Jahren 2004-2005. Asgard-Verl., Sankt Augustin 2006

Goff DC, Posever T, Herz L, Simmons J, Kletti N, Lapierre K, Wilner KD, Law GC, Ko GN (1998): An exploratory haloperidol-controlled dose-finding study of ziprasidone in hospitalized patients with schizophrenia or schizoaffective disorder. J Clin Psychopharmacol $\underline{18}, 296-304$

Goldman M, Tandon R, De Quardo JR, Taylor SF, Goodson J, Mc Grath M (1996): Biological predictors of 1-year outcome in schizophrenia in males and females. Schizophr Res $\underline{21}, 65-73$

Gordin A, Mustajoki P, Pelkonen R (1985): Ketanserin without effects on basal anterior pituitary hormone secretion in healthy subjects. J Endocrinol Invest $\underline{8}, 73-75$

Görtelmeyer R: Schlaffragebogen A und B; in: Internationale Skalen für Psychiatrie; hrsg. v. Collegium Internationale Psychiatriae Scalarum (CIPS); Beltz Test GmbH, Weinheim 1986

Gulevich GD, Dement WC, Zarcone VP (1967): All-night sleep recordings of chronic schizophrenics in remission. Compr Psychiatry $\underline{8}, 141-149$

Gursky JT, Krahn LE (2000): The effects of antidepressants on sleep: a review. Harv Rev Psychiatry $\underline{8}, 298-306$

Hajak G, Rodenbeck A, Ehrenthal HD, Leonard S, Wedekind D, Sengos G, Zhou D, Huether G (1997): No evidence for a physiological coupling between melatonin and glucocorticoids. Psychopharmacology 133, 313-322

Hajak G, Rodenbeck A, Voderholzer U, Riemann D, Cohrs S, Hohagen F, Berger M, Rüther E (2001): Doxepin in the treatment of primary insomnia: a placebo-controlled, doubleblind, polysomnographic study. J Clin Psychiatry $\underline{62}$, 453-463

Hamelin BA, Allard S, Laplante L, Miceli J, Wilner KD, Tremblay J, Le Bel M (1998): The effect of timing of a standard meal on the pharmacokinetics and pharmacodynamics of the novel atypical antipsychotic agent ziprasidone. Pharmacotherapy 18, 9-15 
Harrow M, Yonan CA, Sands JR, Marengo J (1994): Depression in schizophrenia: are neuroleptics, akinesia, or anhedonia involved? Schizophr Bull 20, 327-338

Hartmann E, Cravens J (1973): The effects of long term administration of psychotrophic drugs on human sleep. IV. The effects of chlorpromazine. Psychopharmacologia 33, 203218

Harvey PD, Meltzer H, Simpson GM, Potkin SG, Loebel A, Siu C, Romano SJ (2004a): Improvement in cognitive function following a switch to ziprasidone from conventional antipsychotics, olanzapine, or risperidone in outpatients with schizophrenia. Schizophr Res $\underline{66}, 101-113$

Harvey PD, Siu CO, Romano S (2004b): Randomized, controlled, double-blind, multicenter comparison of the cognitive effects of ziprasidone versus olanzapine in acutely ill inpatients with schizophrenia or schizoaffective disorder. Psychopharmacology 172, 324-332

Hatzimanolis J, Lykouras L, Markianos M, Oulis P (1998): Neurochemical variables in schizophrenic patients during switching from neuroleptics to clozapine. Prog Neuropsychopharmacol Biol Psychiatry 22, 1077-1085

Hemrick-Luecke SK, Evans DC (2002): Comparison of the potency of MDL 100,907 and SB 242084 in blocking the serotonin $\left(5-\mathrm{HT}_{2}\right)$ receptor agonist-induced increases in rat serum corticosterone concentrations: evidence for $5-\mathrm{HT}_{2 \mathrm{~A}}$ receptor mediation of the HPA axis. Neuropharmacology $\underline{42}, 162-169$

Hennig J, Rzepka U, Mai B, Netter P (1995): Suppression of HPA-axis activity by haloperidol after experimentally induced heat stress. Prog Neuropsychopharmacol Biol Psychiatry $\underline{19}, 603-614$

Herz MI, Fava GA, Molnar G, Edwards L (1985): The dexamethasone suppression test in newly hospitalized schizophrenic patients. Am J Psychiatry 142, 127-129

Heuser I, Yassouridis A, Holsboer F (1994): The combined dexamethasone/CRH test: a refined laboratory test for psychiatric disorders. J Psychiatr Res 28 , 341-356

Hinze-Selch D, Mullington J, Orth A, Lauer CJ, Pollmächer T (1997): Effects of clozapine on sleep: a longitudinal study. Biol Psychiatry $\underline{42}$, 260-266

Hobson JA, Pace-Schott EF (2002): The cognitive neuroscience of sleep: neuronal systems, consciousness and learning. Nat Rev Neurosci $\underline{3}, 679-693$

Hobson JA, Mc Carley RW, Wyzinski PW (1975): Sleep cycle oscillation: reciprocal discharge by two brainstem neuronal groups. Science $\underline{189}, 55-58$

Holsboer F (2000): The corticosteroid receptor hypothesis of depression. Neuropsychopharmacology $23,477-501$

Holt RIG, Peveler RC (2006): Association between antipsychotic drugs and diabetes. Diabetes Obes Metab $\underline{8}, 125-135$

Hudson JI, Lipinski JF, Keck PE, Aizley HG, Vuckovic A, Zierk KC, Pope HG (1993): Polysomnographic characteristics of schizophrenia in comparison with mania and depression. Biol Psychiatry $\underline{34}, 191-193$ 
Huether G (1996): The central adaptation syndrome: psychosocial stress as a trigger for adaptive modifications of brain structure and brain function. Prog Neurobiol $\underline{48}, 569-612$

Idzikowski C, Mills FJ, Glennard R (1986): 5-Hydroxytryptamine-2 antagonist increases human slow wave sleep. Brain Res $\underline{378}, 164-168$

Inoué S, Honda K, Komoda Y (1995): Sleep as neuronal detoxification and restitution. Behav Brain Res 69, 91-96

Ismail K, Murray RM, Wheeler MJ, O'Keane V (1998): The dexamethasone suppression test in schizophrenia. Psychol Med 28, 311-317

Jakovljević M, Mück-Seler D, Pivac N, Crncević Z (1998): Platelet 5-HT and plasma cortisol concentrations after dexamethasone suppression test in patients with different time course of schizophrenia. Neuropsychobiology $\underline{37}, 142-145$

Jobert M, Jähnig P, Schulz H (1999): Effect of two antidepressant drugs on REM sleep and EMG activity during sleep. Neuropsychobiology $\underline{39}$, 101-109

Jones JS, Stein DJ, Stanley B, Guido JR, Winchel R, Stanley M (1994): Negative and depressive symptoms in suicidal schizophrenics. Acta Psychiatr Scand $\underline{89}$, 81-87

Kahn RS, Siever L, Davidson M, Greenwald C, Moore C (1993): Haloperidol and clozapine treatment and their effect on M-chlorophenylpiperazine-mediated responses in schizophrenia: implications for the mechanism of action of clozapine. Psychopharmacology $\underline{112}$, 90-94

Kahn RS, Davidson M, Siever LJ, Sevy S, Davis KL (1994): Clozapine treatment and its effect on neuroendocrine responses induced by the serotonin agonist, mchlorophenylpiperazine. Biol Psychiatry $\underline{35}$, 909-912

Kane JM (2003): Oral ziprasidone in the treatment of schizophrenia: a review of short-term trials. J Clin Psychiatry $\underline{64}$, 19-25

Kaplan J, Dawson S, Vaughan T, Green R, Wyatt RJ (1974): Effect of prolonged chlorpromazine administration on the sleep of chronic schizophrenics. Arch Gen Psychiatry $\underline{31}$, $62-66$

Kapur S, Seeman P (2001): Does fast dissociation from the dopamine d(2) receptor explain the action of atypical antipsychotics?: A new hypothesis. Am J Psychiatry 158, 360-369

Kapur S, Mamo D (2003): Half a century of antipsychotics and still a central role for dopamine $\mathrm{D}_{2}$ receptors. Prog Neuropsychopharmacol Biol Psychiatry 27, 1081-1090

Kathol RG, Gehris TL, Carroll BT, Samuelson SD, Pitts AF, Meller WH, Carter JL (1992): Blunted ACTH response to hypoglycemic stress in depressed patients but not in patients with schizophrenia. J Psychiatr Res 26, 103-116

Kempenaers C, Kerkhofs M, Linkowski P, Mendlewicz J (1988): Sleep EEG variables in young schizophrenic and depressive patients. Biol Psychiatry 24

Kerkhofs M, Rielaert C, de Maertelaer V, Linkowski P, Czarka M, Mendlewicz J (1990): Fluoxetine in major depression: efficacy, safety and effects on sleep polygraphic variables. Int Clin Psychopharmacol $\underline{5}, 253-260$ 
Keshavan MS, Miewald J, Haas G, Sweeney J, Ganguli R, Reynolds CF (1995a): Slow-wave sleep and symptomatology in schizophrenia and related psychotic disorders. J Psychiatr Res $\underline{29}, 303-314$

Keshavan MS, Reynolds CF, Miewald J, Montrose D (1995b): Slow-wave sleep deficits and outcome in schizophrenia and schizoaffective disorder. Acta Psychiatr Scand 91, 289-292

Keshavan MS, Reynolds CF, Miewald JM, Montrose DM (1996): A longitudinal study of EEG sleep in schizophrenia. Psychiatry Res 59, 203-211

Keshavan MS, Reynolds CF, Miewald J, Montrose D, Sweeney JA, Vasko RC, Kupfer DJ (1998): Delta sleep deficits in schizophrenia: evidence from automated analyses of sleep data. Arch Gen Psychiatry 55, 443-448

Kupfer DJ (1976): REM latency: a psychobiologic marker for primary depressive disease. Biol Psychiatry 11, 159-174

Kupfer DJ, Foster FG, Reich L, Thompson SK, Weiss B (1976): EEG sleep changes as predictors in depression. Am J Psychiatry $\underline{133}, 622-626$

Kupfer DJ, Coble P, Kane J, Petti T, Conners CK (1979): Imipramine and EEG sleep in children with depressive symptoms. Psychopharmacology $\underline{60}, 117-23$

Laakmann G, Wittmann M, Gugath M, Müller OA, Treusch J, Wahlster U, Stalla GK (1984): Effects of psychotropic drugs (desimipramine, chlorimipramine, sulpiride and diazepam) on the human HPA axis. Psychopharmacology $\underline{84}$, 66-70

Laakmann G, Wittmann M, Schoen HW, Zygan K, Weiss A, Meissner R, Müller OA, Stalla GK (1986): Effects of receptor blockers (methysergide, propranolol, phentolamine, yohimbine and prazosin) on desimipramine-induced pituitary hormone stimulation in humans - III. Hypothalamo-pituitary-adrenocortical axis. Psychoneuroendocrinology 11, $475-489$

Laakmann G, Hinz A, Voderholzer U, Daffner C, Müller OA, Neuhauser H, Neulinger E, Wittmann M (1990): The influence of psychotropic drugs and releasing hormones on anterior pituitary hormone secretion in healthy subjects and depressed patients. Pharmacopsychiatry $23,18-26$

Lammers CH, Garcia-Borreguero D, Schmider J, Gotthardt U, Dettling M, Holsboer F, Heuser IJ (1995): Combined dexamethasone/corticotropin-releasing hormone test in patients with schizophrenia and in normal controls: II. Biol Psychiatry 38, 803-807

Laruelle M, Abi-Dargham A, Van Dyck CH, Gil R, D'Souza CD, Erdos J, McCance E, Rosenblatt W, Fingado C, Zoghbi SS et al. (1996): Single photon emission computerized tomography imaging of amphetamine-induced dopamine release in drug-free schizophrenic subjects. Proc Natl Acad Sci USA 93, 9235-9240

Lauer CJ, Schreiber W, Pollmächer T, Holsboer F, Krieg JC (1997): Sleep in schizophrenia: a polysomnographic study on drug-naive patients. Neuropsychopharmacology $\underline{16}, 51-60$ 
Lee HJ, Kim L, Suh KY (2003): Cognitive deterioration and changes of P300 during total sleep deprivation. Psychiatry Clin Neurosci 57, 490-496

Lee JH, Woo JI, Meltzer HY (2001): Effects of clozapine on sleep measures and sleepassociated changes in growth hormone and cortisol in patients with schizophrenia. Psychiatry Res 103, 157-166

Lee MA, Nash JF, Barnes M, Meltzer HY (1991): Inhibitory effect of ritanserin on the 5-hydroxytryptophan-mediated cortisol, ACTH and prolactin secretion in humans. Psychopharmacology 103, 258-264

Lester BK, Guerrero-Figueroa R (1966): Effects of some drugs on electroencephalographic fast activity and dream time. Psychophysiology 2, 224-236

Lester BK, Coulter JD, Cowden LC, Williams HL (1971): Chlorpromazine and human sleep. Psychopharmacologia 20, 280-287

Lewis SA, Evans JI (1969): Dose effects of chlorpromazine on human sleep. Psychopharmacologia $14,342-348$

Linden M, Thiels C (2001): Epidemiology of prescriptions for neuroleptic drugs: tranquilizers rather than antipsychotics. Pharmacopsychiatry 34, 150-154

López-Muñoz F, Alamo C, Rubio G, Cuenca E (2004): Half a century since the clinical introduction of chlorpromazine and the birth of modern psychopharmacology. Prog Neuropsychopharmacol Biol Psychiatry 28, 205-208

Lucidi F, Devoto A, Violani C, Gennaro LD, Mastracci P, Bertini M (1996): Rapid eye movements density as a measure of sleep need: REM density decreases linearly with the reduction of prior sleep duration. Electroencephalogr Clin Neurophysiol 99, 556-561

Maixner S, Tandon R, Eiser A, Taylor S, De Quardo JR, Shipley J (1998): Effects of Antipsychotic Treatment on Polysomnographic Measures in Schizophrenia: A Replication and Extension. Am J Psychiatry 155, 1600-1602

Mann K, Rossbach W, Müller MJ, Müller-Siecheneder F, Pott T, Linde I, Dittmann RW, Hiemke C (2006): Nocturnal hormone profiles in patients with schizophrenia treated with olanzapine. Psychoneuroendocrinology 31, 256-264

Maquet P (2001): The role of sleep in learning and memory. Science 294, 1048-1052

Marco EJ, Wolkowitz OM, Vinogradov S, Poole JH, Lichtmacher J, Reus VI (2002): Doubleblind antiglucocorticoid treatment in schizophrenia and schizoaffective disorder: a pilot study. World J Biol Psychiatry $\underline{3}, 156-161$

Marder SR (2006): A review of agitation in mental illness: treatment guidelines and current therapies. J Clin Psychiatry 67, 13-21

Markianos M, Hatzimanolis J, Lykouras L (1999a): Gonadal axis hormones in male schizophrenic patients during treatment with haloperidol and after switch to risperidone. Psychopharmacology 143, 270-272 
Markianos M, Hatzimanolis J, Lykouras L (1999b): Switch from neuroleptics to clozapine does not influence pituitary-gonadal axis hormone levels in male schizophrenic patients. Eur Neuropsychopharmacol $\underline{9}, 533-536$

Mayers AG, Baldwin DS (2005): Antidepressants and their effect on sleep. Hum Psychopharmacol 20, 533-559

Mc Ewen BS: Stress and neuroendocrine funktion. Individual differences and mechanisms leading to disease; in: Psychoneuroendocrinology. The Scientific Basis of Clinical Practice; hrsg. v. Wolkowitz OM, Rothschild AJ; American Psychiatrist Publishing, Inc., Arlington 2003, 513-546

Mc Ginty D, Szymusiak R (1990): Keeping cool: a hypothesis about the mechanisms and functions of slow-wave sleep. Trends Neurosci 13, 480-487

Meltzer HY (1989): Clinical studies on the mechanism of action of clozapine: the dopamineserotonin hypothesis of schizophrenia. Psychopharmacology 99, 18-27

Meltzer HY (1999): The role of serotonin in antipsychotic drug action. Neuropsychopharmacology $21,106-115$

Miceli JJ, Wilner KD, Hansen RA, Johnson AC, Apseloff G, Gerber N (2000): Single- and multiple-dose pharmacokinetics of ziprasidone under non-fasting conditions in healthy male volunteers. Br J Clin Pharmacol 49, 5-13

Miller DD (2004): Atypical antipsychotics: sleep, sedation, and efficacy. Prim Care Companion J Clin Psychiatry $\underline{6}, 3-7$

Minas IH, Jackson HJ, Joshua SD, Burgess PM (1990): Depression, negative and positive symptoms, and the DST in schizophrenia. Schizophr Res $\underline{3}, 321-327$

Möller HJ (2003): Management of the negative symptoms of schizophrenia: new treatment options. CNS Drugs 17, 793-823

Möller HJ, Kissling W, Bottermann P (1986): The dexamethasone suppression test in depressive and schizophrenic patients under controlled treatment conditions. Eur Arch Psychiatry Neurol Sci 235, 263-268

Möller HJ, Laux G, Deister A, Braun-Scharm H: Psychiatrie und Psychotherapie. 2. Auflage; Thieme, Stuttgart 2001

Monteleone P, Maj M, Fusco M, Kemali D, Reiter RJ (1992): Depressed nocturnal plasma melatonin levels in drug-free paranoid schizophrenics. Schizophr Res $\underline{7}, 77-84$

Monteleone P, Tortorella A, Borriello R, Cassandro P, Maj M (1999): Prolactin hyperresponsiveness to D-fenfluramine in drug-free schizophrenic patients: a placebo-controlled study. Biol Psychiatry 45, 1606-1611

Monti JM (1989): Effect of a reversible monoamine oxidase-A inhibitor (moclobemide) on sleep of depressed patients. Br J Psychiatry 155, 61-65

Monti JM, Monti D (2004): Sleep in schizophrenia patients and the effects of antipsychotic drugs. Sleep Med Rev $\underline{8}, 133-148$ 
Monti JM, Monti D (2005): Sleep disturbance in schizophrenia. Int Rev Psychiatry 17, $247-253$

Mossman D, Somoza E (1989): Maximizing diagnostic information from the dexamethasone suppression test. An approach to criterion selection using receiver operating characteristic analysis. Arch Gen Psychiatry 46, 653-660

Mouret J, Lemoine P, Minuit MP, Sanchez P, Taillard J (1989): Sleep polygraphic effects of trimipramine in depressed patients. Preliminary report. Drugs $\underline{38}, 14-16$

Mück-Seler D, Pivac N, Jakovljević M, Brzović Z (1999): Platelet serotonin, plasma cortisol, and dexamethasone suppression test in schizophrenic patients. Biol Psychiatry 45, 14331439

Müller MJ, Rossbach W, Mann K, Röschke J, Müller-Siecheneder F, Blümler M, Wetzel H, Russ H, Dittmann RW, Benkert O (2004): Subchronic effects of olanzapine on sleep EEG in schizophrenic patients with predominantly negative symptoms. Pharmacopsychiatry 37, $157-162$

Müller P, Kind J, Steuber H (1978): Pharmakogene depressive Syndrome im Verlauf schizophrener Psychosen. Arzneimittelforschung 28, 1501-1502

Munro JG, Hardiker TM, Leonard DP (1984): The dexamethasone suppression test in residual schizophrenia with depression. Am J Psychiatry 141, 250-252

Murburg MM, Paly D, Wilkinson CW, Veith RC, Malas KL, Dorsa DM (1986): Haloperidol increases plasma beta endorphin-like immunoreactivity and cortisol in normal human males. Life Sci 39, 373-381

Murburg MM, Wilkinson CW, Raskind MA, Veith RC, Dorsa DM (1993): Evidence for two differentially regulated populations of peripheral beta-endorphin-releasing cells in humans. J Clin Endocrinol Metab 77, 1033-1040

Naber D, Albus M, Bürke H, Müller-Spahn F, Münch U, Reinertshofer T, Wissmann J, Ackenheil M (1985): Neuroleptic withdrawal in chronic schizophrenia: CT and endocrine variables relating to psychopathology. Psychiatry Res 16, 207-219

Nakazawa Y, Kotorii M, Kotorii T, Ohshima M, Hasuzawa H (1977): Individual variations in response of human REM sleep to amitriptyline and haloperidol. Electroencephalogr Clin Neurophysiol $\underline{42}, 769-775$

Nemeroff CB (2005): Use of atypical antipsychotics in refractory depression and anxiety. J Clin Psychiatry 66, 13-21

Newcomer JW, Faustman WO, Whiteford HA, Moses JA, Csernansky JG (1991): Symptomatology and cognitive impairment associate independently with post-dexamethasone cortisol concentrations in unmedicated schizophrenic patients. Biol Psychiatry 29, 855864

Newcomer JW, Craft S, Askins K, Hershey T, Bardgett ME, Csernansky JG, Gagliardi AE, Vogler G (1998): Glucocorticoid interactions with memory function in schizophrenia. Psychoneuroendocrinology $\underline{23}, 65-72$ 
Newcomer JW, Selke G, Melson AK, Hershey T, Craft S, Richards K, Alderson AL (1999): Decreased memory performance in healthy humans induced by stress-level cortisol treatment. Arch Gen Psychiatry 56, 527-533

Newcomer JW, Haupt DW, Fucetola R, Melson AK, Schweiger JA, Cooper BP, Selke G (2002): Abnormalities in glucose regulation during antipsychotic treatment of schizophrenia. Arch Gen Psychiatry 59, 337-345

Neylan TC, van Kammen DP, Kelley ME, Peters JL (1992): Sleep in schizophrenic patients on and off haloperidol therapy. Clinically stable vs relapsed patients. Arch Gen Psychiatry $\underline{49}, 643-649$

Nofzinger EA, van Kammen DP, Gilbertson MW, Gurklis JA, Peters JL (1993): Electroencephalographic sleep in clinically stable schizophrenic patients: two-weeks versus six-weeks neuroleptic-free. Biol Psychiatry 33, 829-835

Nofzinger EA, Reynolds CF, Thase ME, Frank E, Jennings JR, Fasiczka AL, Sullivan LR, Kupfer DJ (1995): REM sleep enhancement by bupropion in depressed men. Am J Psychiatry $\underline{152}, 274-276$

Nowell PD, Buysse DJ, Hall M, Reynolds CF: Psychometric and psychiatric evaluation; in: Principles and practice of sleep medicine; hrsg. v. Kryger MH, Roth T, Dement WC; W. B. Saunders Company, Philadelphia 2000, 1290-1294

O'Dwyer AM, Lightman SL, Marks MN, Checkley SA (1995): Treatment of major depression with metyrapone and hydrocortisone. J Affect Disord 33, 123-128

Okuma T, Hata N, Fujii S (1975): Differential effects of chlorpromazine, imipramine, nitrazepam and amobarbital on REM sleep and REM density in man. Folia Psychiatr Neurol Jpn $\underline{29}, 25-37$

Ott H, Oswald I, Fichte K, Sastre-Y-Hernández M: Visuelle Analogskalen zur Erfassung von Schlafqualität; in: Internationale Skalen für Psychiatrie; hrsg. v. Collegium Internationale Psychiatriae Scalarum (CIPS); Beltz Test GmbH, Weinheim 1986

Owen RR, Gutierrez-Esteinou R, Hsiao J, Hadd K, Benkelfat C, Lawlor BA, Murphy DL, Pickar D (1993): Effects of clozapine and fluphenazine treatment on responses to mchlorophenylpiperazine infusions in schizophrenia. Arch Gen Psychiatry 50, 636-644

Pace-Schott EF, Hobson JA (2002): The neurobiology of sleep: genetics, cellular physiology and subcortical networks. Nat Rev Neurosci $\underline{3}, 591-605$

Paiva T, Arriaga F, Wauquier A, Lara E, Largo R, Leitao JN (1988): Effects of ritanserin on sleep disturbances of dysthymic patients. Psychopharmacology 96, 395-399

Papakostas GI, Petersen TJ, Nierenberg AA, Murakami JL, Alpert JE, Rosenbaum JF, Fava M (2004): Ziprasidone augmentation of selective serotonin reuptake inhibitors (SSRIs) for SSRI-resistant major depressive disorder. J Clin Psychiatry 65, 217-221

Penzel T, Hajak G, Hoffmann RM, Lund R, Podszus T, Pollmächer T, Schäfer T, Schulz H, Sonnenschein W, Spieweg I (1993): Empfehlungen zur Durchführung und Auswer- 
tung polygraphischer Ableitungen im diagnostischen Schlaflabor. Z Elektroenzephalogr Elektromyogr Verwandte Geb 24, 65-70

Pfizer, Fachinformation Zeldox ${ }^{\circledR}$ Hartkapseln. Rote Liste ${ }^{\circledR}$ Service GmbH, Frankfurt/Main 2006

Pinto LR, Peres CA, Russo RH, Remesar-Lopez AJ, Tufik S (2002): Sawtooth waves during REM sleep after administration of haloperidol combined with total sleep deprivation in healthy young subjects. Braz J Med Biol Res 35, 599-604

Plihal W, Born J (1997): Effects of early and late nocturnal sleep on declarative and procedural memory. J Cogn Neurosci $\underline{9}, 534-547$

Plocka-Lewandowska M, Araszkiewicz A, Rybakowski JK (2001): Dexamethasone suppression test and suicide attempts in schizophrenic patients. Eur Psychiatry 16, 428-431

Puca FM, Dammacco F, Rigillo N, Specchio LM, Genco S, Chetrì G, Torelli A, Maggiore A, Frezza E, Leomanni R (1978): Increzione somatotropinica ed EEG durante il sonno notturno dell' adulto normale dopo pimozide. Boll Soc Ital Biol Sper 54, 460-465

Rao ML, Strebel B, Halaris A, Gross G, Bräunig P, Huber G, Marler M (1995): Circadian rhythm of vital signs, norepinephrine, epinephrine, thyroid hormones, and cortisol in schizophrenia. Psychiatry Res $\underline{57}, 21-39$

Rao U, Lin KM, Schramm P, Poland RE (2004): REM sleep and cortisol responses to scopolamine during depression and remission in women. Int J Neuropsychopharmacol $\underline{7}$, $265-274$

Rechtschaffen A, Kales A, A manual of standardized terminology, techniques and scoring system for sleep stages of human subjects. U. S. Government Printing Office, Washington D. C. 1968

Reynolds CF, Buysse DJ, et al. (1997): Maintenance nortriptyline effects on electroencephalographic sleep in elderly patients with recurrent major depression: double-blind, placeboand plasma-level-controlled evaluation. Biol Psychiatry $\underline{42}, 560-567$

Richelson E (1999): Receptor pharmacology of neuroleptics: relation to clinical effects. J Clin Psychiatry $\underline{60}, 5-14$

Richelson E, Souder T (2000): Binding of antipsychotic drugs to human brain receptors focus on newer generation compounds. Life Sci 68 , 29-39

Riech SS, Repetitive transkranielle Magnetstimulation: Die Auswirkungen auf den Schlaf und die Stimmung. Med. Diss. Göttingen 2002

Riemann D, Velthaus S, Laubenthal S, Müller WE, Berger M (1990): REM-suppressing effects of amitriptyline and amitriptyline-N-oxide after acute medication in healthy volunteers: results of two uncontrolled pilot trials. Pharmacopsychiatry $\underline{23}$, 253-258

Riemann D, Berger M, Voderholzer U (2001): Sleep and depression - results from psychobiological studies: an overview. Biol Psychol 57, 67-103 
Riemann D, Voderholzer U, Cohrs S, Rodenbeck A, Hajak G, Rüther E, Wiegand MH, Laakmann G, Baghai T, Fischer W et al. (2002): Trimipramine in primary insomnia: results of a polysomnographic double-blind controlled study. Pharmacopsychiatry 35 , $165-174$

Rijnbeek B, de Visser SJ, Franson KL, Cohen AF, van Gerven JMA (2003): REM sleep effects as a biomarker for the effects of antidepressants in healthy volunteers. J Psychopharmacol 17, 196-203

Risberg AM, Risberg J, Ingvar DH (1975): Effects of promethazine on nocturnal sleep in normal man. Psychopharmacologia $\underline{43}$, 279-284

Ritsner M, Kurs R, Ponizovsky A, Hadjez J (2004): Perceived quality of life in schizophrenia: relationships to sleep quality. Qual Life Res $\underline{13}$, 783-791

Rodenbeck A, Binder R, Geisler P, Danker-Hopfe H, Lund R, Raschke F, Weeß HG, Schulz H (2006): A Review of Sleep EEG Patterns. Part I: A Compilation of Amended Rules for Their Visual Recognition according to Rechtschaffen and Kales. Somnologie 10, 159-175

Rothschild AJ: The hypothalamic-pituitary-adrenal axis and psychiatric illness; in: Psychoneuroendocrinology. The Scientific Basis of Clinical Practice; hrsg. v. Wolkowitz OM, Rothschild AJ; American Psychiatrist Publishing, Inc., Arlington 2003, 139-163

Rüther E, Davis L, Papousek M, Reichinger M, Reiter H, Rudolph M (1976): Pharmakologische Beeinflussung zentraler serotonerger Mechanismen am Menschen und Auswirkungen auf den Schlaf. Arzneimittelforschung 26, 1071-1073

Rush AJ, Armitage R, et al. (1998): Comparative effects of nefazodone and fluoxetine on sleep in outpatients with major depressive disorder. Biol Psychiatry $\underline{44}, 3-14$

Ryan MCM, Collins P, Thakore JH (2003): Impaired fasting glucose tolerance in firstepisode, drug-naive patients with schizophrenia. Am J Psychiatry 160, 284-289

Ryan MCM, Flanagan S, Kinsella U, Keeling F, Thakore JH (2004): The effects of atypical antipsychotics on visceral fat distribution in first episode, drug-naive patients with schizophrenia. Life Sci 74, 1999-2008

Sachar EJ, Hellman L, Fukushima DK, Gallagher TF (1970): Cortisol production in depressive illness. A clinical and biochemical clarification. Arch Gen Psychiatry 23, 289-298

Sagalés T, Erill S (1975): Effects of central dopaminergic blockade with primozide upon the EEG stages of sleep in man. Psychopharmacologia 41, 53-56

Saldanha VF, Havard CW, Bird R, Gardner R (1972): The effect of chlorpromazine on pituitary function. Clin Endocrinol (Oxf) 1, 173-180

Salin-Pascual RJ, Herrera-Estrella M, Galicia-Polo L, Laurrabaquio MR (1999): Olanzapine acute administration in schizophrenic patients increases delta sleep and sleep efficiency. Biol Psychiatry $\underline{46}, 141-143$

Sapolsky RM, Uno H, Rebert CS, Finch CE (1990): Hippocampal damage associated with prolonged glucocorticoid exposure in primates. J Neurosci 10, 2897-2902 
Sauro MD, Jorgensen RS, Pedlow CT (2003): Stress, glucocorticoids, and memory: a metaanalytic review. Stress $\underline{6}, 235-245$

Sawyer J, Jeffries JJ (1984): The dexamethasone suppression test in schizophrenia. J Clin Psychiatry $\underline{45}$, 399-402

Scheepers FE, De Wied CCG, Westenberg HG, Kahn RS (2001): The effect of olanzapine treatment on monoamine metabolite concentrations in the cerebrospinal fluid of schizophrenic patients. Neuropharmacology 25, 468-475

Scherk H, Falkai P (2006): Effects of antipsychotics on brain structure. Curr Opin Psychiatry 19, $145-150$

Schmidt AW, Lebel LA, Howard HR Jr, Zorn SH (2001): Ziprasidone: a novel antipsychotic agent with a unique human receptor binding profile. Eur J Pharmacol 425, 197-201

Schoemaker H, Claustre Y, et al. (1997): Neurochemical characteristics of amisulpride, an atypical dopamine $\mathrm{D}_{2} / \mathrm{D}_{3}$ receptor antagonist with both presynaptic and limbic selectivity. J Pharmacol Exp Ther 280, 83-97

Seeger TF, Seymour PA, et al. (1995): Ziprasidone (CP-88,059): A New Antipsychotic with Combined Dopamine and Serotonin Receptor Antagonist Activity. J Pharmacol Exp Ther $275,101-113$

Seeman P (2002): Atypical antipsychotics: mechanism of action. Can J Psychiatry 47, 27-38

Seeman TE, Mc Ewen BS, Singer BH, Albert MS, Rowe JW (1997): Increase in urinary cortisol excretion and memory declines: MacArthur studies of successful aging. J Clin Endocrinol Metab 2, 2458-2465

Seibyl JP, Krystal JH, Price LH, Woods SW, D'Amico C, Heninger GR, Charney DS (1991): Effects of ritanserin on the behavioral, neuroendocrine, and cardiovascular responses to meta-chlorophenylpiperazine in healthy human subjects. Psychiatry Res $\underline{38}, 227-236$

Sharpley AL, Cowen PJ (1995): Effect of pharmacologic treatments on the sleep of depressed patients. Biol Psychiatry $37,85-98$

Sharpley AL, Elliott JM, Attenburrow MJ, Cowen PJ (1994): Slow wave sleep in humans: role of $5-\mathrm{HT}_{2 \mathrm{~A}}$ and $5-\mathrm{HT}_{2 \mathrm{C}}$ receptors. Neuropharmacology $33,467-471$

Sharpley AL, Williamson DJ, Attenburrow ME, Pearson G, Sargent P, Cowen PJ (1996): The effects of paroxetine and nefazodone on sleep: a placebo controlled trial. Psychopharmacology $\underline{126}, 50-54$

Sharpley AL, Vassallo CM, Cowen PJ (2000): Olanzapine increases slow-wave sleep: evidence for blockade of central 5- $\mathrm{HT}_{2 \mathrm{C}}$ receptors in vivo. Biol Psychiatry $\underline{47}, 468-470$

Sharpley AL, Vassallo CM, Pooley EC, Harrison PJ, Cowen PJ (2001): Allelic variation in the $5-\mathrm{HT}_{2 \mathrm{C}}$ receptor $(\mathrm{HT} 2 \mathrm{RC})$ and the increase in slow wave sleep produced by olanzapine. Psychopharmacology 153, 271-272

Sharpley AL, Bhagwagar Z, Hafizi S, Whale WR, Gijsman HJ, Cowen PJ (2003): Risperidone augmentation decreases rapid eye movement sleep and decreases wake in treatmentresistant depressed patients. J Clin Psychiatry $\underline{64}$, 192-196 
Shelton RC, Tollefson GD, Tohen M, Stahl S, Gannon KS, Jacobs TG, Buras WR, Bymaster FP, Zhang W, Spencer KA et al. (2001): A novel augmentation strategy for treating resistant major depression. Am J Psychiatry 158, 131-134

Shirayama Y, Hashimoto K, Suzuki Y, Higuchi T (2002): Correlation of plasma neurosteroid levels to the severity of negative symptoms in male patients with schizophrenia. Schizophr Res $\underline{58}, 69-74$

Smith C (2001): Sleep states and memory processes in humans: procedural versus declarative memory systems. Sleep Med Rev $\underline{5}, 491-506$

Smith C, MacNeill C (1994): Impaired motor memory for a pursuit rotor task following Stage 2 sleep loss in college students. J Sleep Res $\underline{3}, 206-213$

Sora I, Nishimon K, Otsuki S (1986): Dexamethasone suppression test and noradrenergic function in affective and schizophrenic disorders. Biol Psychiatry 21, 621-631

Stahl SM, Shayegan DK (2003): The psychopharmacology of ziprasidone: receptor-binding properties and real-world psychiatric practice. J Clin Psychiatry $\underline{64}, 6-12$

Starkman MN, Gebarski SS, Berent S, Schteingart DE (1992): Hippocampal formation volume, memory dysfunction, and cortisol levels in patients with Cushing's syndrome. Biol Psychiatry $\underline{32}, 756-765$

Tandon R, Silk KR, Greden JF, Goodson J, Hariharan M, Meador-Woodruff JH, Kronfol Z (1989): Positive and negative symptoms in schizophrenia and the Dexamethasone Suppression Test. Biol Psychiatry 25, 788-792

Tandon R, Mazzara C, De Quardo J, Craig KA, Meador-Woodruff JH, Goldman R, Greden JF (1991): Dexamethasone suppression test in schizophrenia: relationship to symptomatology, ventricular enlargement, and outcome. Biol Psychiatry 29, 953-964

Tandon R, Shipley JE, Taylor S, Greden JF, Eiser A, De Quardo J, Goodson J (1992): Electroencephalographic sleep abnormalities in schizophrenia. Relationship to positive/negative symptoms and prior neuroleptic treatment. Arch Gen Psychiatry 499, 185-194

Tandon R, De Quardo JR, Taylor SF, Mc Grath M, Jibson M, Eiser A, Goldman M (2000): Phasic and enduring negative symptoms in schizophrenia: biological markers and ralationship to outcome. Schizophr Res $\underline{45}, 191-201$

Tatsumi M, Jansen K, Blakely RD, Richelson E (1999): Pharmacological profile of neuroleptics at human monoamine transporters. Eur J Pharmacol 368, 277-283

Taylor SF, Tandon R, Shipley JE, Eiser AS, Goodson J (1991): Sleep onset REM periods in schizophrenic patients. Biol Psychiatry 30, 205-209

Tepavcević D, Giljević Z, Korsić M, Halimi S, Suchanek E, Jelić T, Aganović I, Kozić B, Plavsić V (1994): Effects of ritanserin, a novel serotonin-S2 receptor antagonist, on the secretion of pituitary hormones in normal humans. J Endocrinol Invest 17, 1-5

Tepavcević D, Giljević Z, Aganović I, Korsić M, Halimi S, Suchanek E, Jelić T, Kozić B, Plavsić V (1995): Effects of ritanserin, a specific serotonin-S2 receptor antagonist, on the 
release of anterior pituitary hormones during insulin-induced hypoglycemia in normal humans. J Endocrinol Invest 18, 427-430

Thaker GK, Wagman AM, Kirkpatrick B, Tamminga CA (1989): Alterations in sleep polygraphy after neuroleptic withdrawal: a putative supersensitive dopaminergic mechanism. Biol Psychiatry 25, 75-86

Thakore JH, Mann JN, Vlahos I, Martin A, Reznek R (2002): Increased visceral fat distribution in drug-naive and drug-free patients with schizophrenia. Int J Obes Relat Metab Disord 26, 137-141

Tohen M, Vieta E, et al. (2003): Efficacy of olanzapine and olanzapine-fluoxetine combination in the treatment of bipolar I depression. Arch Gen Psychiatry $\underline{60}$, 1079-1088

Touyz SW, Beumont PJ, Saayman GS, Zabow T (1977): A psychophysiological investigation of the short-term effects of clozapine upon sleep parameters of normal young adults. Biol Psychiatry 12 , 801-822

Touyz SW, Saayman GS, Zabow T (1978): A psychophysiological investigation of the longterm effects of clozapine upon sleep patterns of normal young adults. Psychopharmacology $\underline{56}, 69-73$

Trivedi MH, Rush AJ, Armitage R, Gullion CM, Grannemann BD, Orsulak PJ, Roffwarg HP (1999): Effects of fluoxetine on the polysomnogram in outpatients with major depression. Neuropsychopharmacology $20,447-459$

Van de Kar LD, Javed A, Zhang Y, Serres F, Raap DK, Gray TS (2001): 5-HT 2 A receptors stimulate ACTH, corticosterone, oxytocin, renin, and prolactin release and activate hypothalamic CRF and oxytocin-expressing cells. J Neurosci 21, 3572-3579

Van Dongen HPA, Maislin G, Mullington JM, Dinges DF (2003): The cumulative cost of additional wakefulness: dose-response effects on neurobehavioral functions and sleep physiology from chronic sleep restriction and total sleep deprivation. Sleep 26, 117-126

Van Kammen DP, Van Kammen WB, Peters JL, Rosen J, SR C, Neylan T, Linnoila M (1986): CSF MHPG, sleep and psychosis in schizophrenia. Clin Neuropharmacol 9, 575577

Varghese FP, Brown E (2001): The Hypothalamic-Pituitary-Adrenal Axis in Major Depressive Disorder: A Brief Primer for Primary Care Physicians. Prim Care Companion J Clin Psychiatry $\underline{3}, 151-155$

Velligan DI, Prihoda TJ, Sui D, Ritch JL, Maples N, Miller AL (2003): The effectiveness of quetiapine versus conventional antipsychotics in improving cognitive and functional outcomes in standard treatment settings. J Clin Psychiatry $\underline{64}, 524-531$

Vogel GW (1975): A review of REM sleep deprivation. Arch Gen Psychiatry 32, 749-761

Vogel GW, Thurmond A, Gibbons P, Sloan K, Walker M (1975): REM sleep reduction effects on depression syndromes. Arch Gen Psychiatry 32, 765-777 
Von Bahr C, Wiesel FA, Movin G, Eneroth P, Jansson P, Nilsson L, Ogenstad S (1991): Neuroendocrine responses to single oral doses of remoxipride and sulpiride in healthy female and male volunteers. Psychopharmacology 103, 443-448

Vythilingam M, Vermetten E, Anderson GM, Luckenbaugh D, Anderson ER, Snow J, Staib LH, Charney DS, Bremner JD (2004): Hippocampal volume, memory, and cortisol status in major depressive disorder: effects of treatment. Biol Psychiatry 56, 101-112

Walder DJ, Walker EF, Lewine RJ (2000): Cognitive functioning, cortisol release, and symptom severity in patients with schizophrenia. Biol Psychiatry $\underline{48}, 1121-1132$

Waldo MC, Cawthra E, Adler LE, Dubester S, Staunton M, Nagamoto H, Baker N, Madison A, Simon J, Scherzinger A (1994): Auditory sensory gating, hippocampal volume, and catecholamine metabolism in schizophrenics and their siblings. Schizophr Res $\underline{12}, 93-106$

Walker EF, Diforio D (1997): Schizophrenia: a neural diathesis-stress model. Psychol Rev 104, 667-685

Walker MP, Stickgold R (2006): Sleep, memory, and plasticity. Annu Rev Psychol 57, 139166

Walker MP, Brakefield T, Morgan A, Hobson JA, Stickgold R (2002): Practice with sleep makes perfect: sleep-dependent motor skill learning. Neuron 35, 205-211

Ware JC, Brown FW, Moorad PJ, Pittard JT, Cobert B (1989): Effects on sleep: a doubleblind study comparing trimipramine to imipramine in depressed insomniac patients. Sleep $\underline{12}, 537-549$

Warrington TP, Bostwick JM (2006): Psychiatric adverse effects of corticosteroids. Mayo Clin Proc $\underline{81}$, 1361-1367

Wehr TA, Wirz-Justice A, Goodwin FK, Duncan W, Gillin JC (1979): Phase advance of the circadian sleep-wake cycle as an antidepressant. Science 206, 710-713

Weinstein DD, Diforio D, Schiffman J, Walker E, Bonsall R (1999): Minor physical anomalies, dermatoglyphic asymmetries, and cortisol levels in adolescents with schizotypal personality disorder. Am J Psychiatry 156, 617-623

Wetter TC, Lauer CJ, Gillich G, Pollmächer T (1996): The electroencephalographic sleep pattern in schizophrenic patients treated with clozapine or classical antipsychotic drugs. J Psychiatr Res 30, 411-419

Wetzel H, Wiesner J, Hiemke C, Benkert O (1994): Acute antagonism of dopamine $\mathrm{D}_{2}$-like receptors by amisulpride: effects on hormone secretion in healthy volunteers. J Psychiatr Res $\underline{28}, 461-473$

Wik G (1995): Effects of neuroleptic treatment on cortisol and 3-methoxy4-hydroxyphenylethyl glycol levels in blood. J Endocrinol 144, 425-429

Wik G, Wiesel FA, Eneroth P, Sedvall G, Aström G (1986): Dexamethasone suppression test in schizophrenic patients before and during neuroleptic treatment. Acta Psychiatr Scand $\underline{74}, 161-167$ 
Wilson S, Argyropoulos S (2005): Antidepressants and sleep: a qualitative review of the literature. Drugs $\underline{65}, 927-947$

Winokur A, Gary KA, Rodner S, Rae-Red C, Fernando AT, Szuba MP (2001): Depression, sleep physiology, and antidepressant drugs. Depress Anxiety 14, 19-28

Wolkowitz OM, Reus VI (1999): Treatment of depression with antiglucocorticoid drugs. Psychosom Med 61, 698-711

Wuttke W: Endokrinologie; in: Physiologie des Menschen; hrsg. v. Schmidt RF, Thews G; Springer, Berlin 1997, 370-407

Yamashita H, Morinobu S, Yamawaki S, Horiguchi J, Nagao M (2002): Effect of risperidone on sleep in schizophrenia: a comparison with haloperidol. Psychiatry Res 109, 137-142

Yeragani VK (1990): The incidence of abnormal dexamethasone suppression in schizophrenia: a review and a meta-analytic comparison with the incidence in normal controls. Can J Psychiatry 35, 128-132

Yoshida T, Mashimo S, Mio M, Kamei C (1997): Histamine-induced cortisol secretion from bovine adrenocortical cells: co-incubated with bovine adrenal medullary cells. Jpn J Pharmacol $\underline{75}, 115-121$

Young AH, Gallagher P, Watson S, Del-Estal D, Owen BM, Ferrier IN (2004): Improvements in neurocognitive function and mood following adjunctive treatment with mifepristone (RU-486) in bipolar disorder. Neuropsychopharmacology 29, 1538-1545

Zarcone VP, Benson KL (1997): BPRS symptom factors and sleep variables in schizophrenia. Psychiatry Res $\underline{66}, 111-120$

Zarcone VP, Benson KL, Berger PA (1987): Abnormal rapid eye movement latencies in schizophrenia. Arch Gen Psychiatry 44, 45-48

Zhang XY, Zhou DF, Cao LY, Wu GY, Shen YC (2005): Cortisol and cytokines in chronic and treatment-resistant patients with schizophrenia: association with psychopathology and response to antipsychotics. Neuropsychopharmacology 30, 1532-1538

Zoccali C, Zabludowski JR, Isles CG, Murray GD, Inglis GC, Robertson JI, Fraser R, Ball SG (1983): The effect of a 5-HT antagonist, ketanserin, on blood pressure, the reninangiotensin system and sympathoadrenal function in normal man. Br J Clin Pharmacol $\underline{16}, 305-311$ 


\section{Danksagung}

Besonders bedanke ich mich bei Herrn Priv.-Doz. Dr. med. S. Cohrs für die Überlassung des interessanten Themas, für die sehr gute Betreuung sowie für die Bereitschaft zu konstruktiver und gewinnbringender Kritik. Sein Interesse an dem Projekt und an der Fertigstellung der Ergebnisse hat entscheidend zu der Verwirklichung dieser Arbeit beigetragen. Frau Priv.-Doz. Dr. rer. nat. Rodenbeck danke ich für die Unterstützung bei den statistischen Berechnungen.

Allen Mitarbeitern des Schlaflabors danke ich für den Beistand während der Durchführung meiner Studie. Insbesondere danke ich Herrn Andreas Müller-Struck für die sehr gute Einarbeitung und die kompetente Hilfe bei allen technischen Fragestellungen. Frau Roswitha Bianco, Brigitte Marxen und Iris Bossmann möchte ich ferner für ihre Unterstützung bei der Betreuung der Probanden danken. Mein herzlicher Dank gilt außerdem Frau Uta Engelhardt aus dem Neurobiologischen Labor.

An dieser Stelle möchte ich mich auch bei allen Probanden bedanken, ohne sie wäre die Durchführung dieser Studie nicht möglich gewesen. Von gegenseitiger Motivation und von inhaltlichem Austausch mit meiner Mitdoktorandin Frau Dr. med. dent. Cornelia Röher habe ich sehr profitiert. Frau Dr. med. Katrin Radenbach danke ich für Korrekturen und Anmerkungen während des Schreibens dieser Arbeit. Ganz besonders bedanke ich mich bei Dr. rer. nat. Jörg Hahn für seine wertvollen Ratschläge,

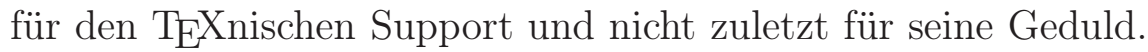




\section{Lebenslauf}

Am 18.08.1976 wurde ich als Kind von Hildegard Neumann und Dr. rer. nat. Wilhelm Rode geboren. In Norden/Ostfriesland besuchte ich von 1983-1986 die Grundschule und im Anschluss 1986-1989 die Orientierungsstufe. 1996 schloss ich meine schulische Ausbildung mit der allgemeinen Hochschulreife am Ulrichsgymnasium Norden ab.

Im Herbst 1996 begann ich das Studium der Humanmedizin an der Georg-AugustUniversität in Göttingen. Die ärztliche Vorprüfung bestand ich 1998, 1999 folgte der erste Abschnitt der ärztlichen Prüfung. Von September 2000 bis September 2001 studierte ich Medizin an der Universität Århus in Dänemark. Nach dem zweiten Abschnitt der ärztlichen Prüfung im Sommer 2002 absolvierte ich das Praktische Jahr im Südharzkrankenhaus Nordhausen. Im Oktober 2003 schloss ich mit der ärztlichen Prüfung das Medizinstudium an der Universität Göttingen ab.

Im Februar 2004 begann ich meine Doktorarbeit bei Herrn Priv.-Doz. Dr. S. Cohrs im Schlaflabor der Abteilung Psychiatrie und Psychotherapie des Universitätsklinikums Göttingen.

Von August 2004 war ich als Ärztin im Praktikum und ab Oktober 2004 bis Dezember 2005 als Assistenzärztin in der Abteilung für Psychiatrie und Psychotherapie des Universitätsklinikums tätig.

Nach der Geburt meines Sohnes Linus Albin im Dezember 2005 befinde ich mich noch in der Elternzeit. 